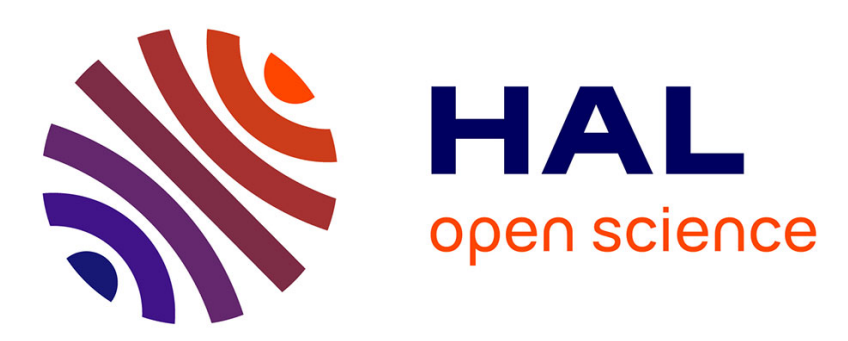

\title{
Catalogue des types de la collection de phasmes du Muséum national d'Histoire naturelle de Paris (Insecta, Phasmatodea)
}

Emmanuel Delfosse, Nicolas Cliquenois, Marion Depraetere, Tony Robillard

\section{- To cite this version:}

Emmanuel Delfosse, Nicolas Cliquenois, Marion Depraetere, Tony Robillard. Catalogue des types de la collection de phasmes du Muséum national d'Histoire naturelle de Paris (Insecta, Phasmatodea). Zoosystema, 2019, 41 (11), pp.181-235. 10.5252/zoosystema2019v41a11 . hal-02614621

\section{HAL Id: hal-02614621 \\ https://hal.science/hal-02614621}

Submitted on 8 Jun 2020

HAL is a multi-disciplinary open access archive for the deposit and dissemination of scientific research documents, whether they are published or not. The documents may come from teaching and research institutions in France or abroad, or from public or private research centers.
L'archive ouverte pluridisciplinaire HAL, est destinée au dépôt et à la diffusion de documents scientifiques de niveau recherche, publiés ou non, émanant des établissements d'enseignement et de recherche français ou étrangers, des laboratoires publics ou privés. 


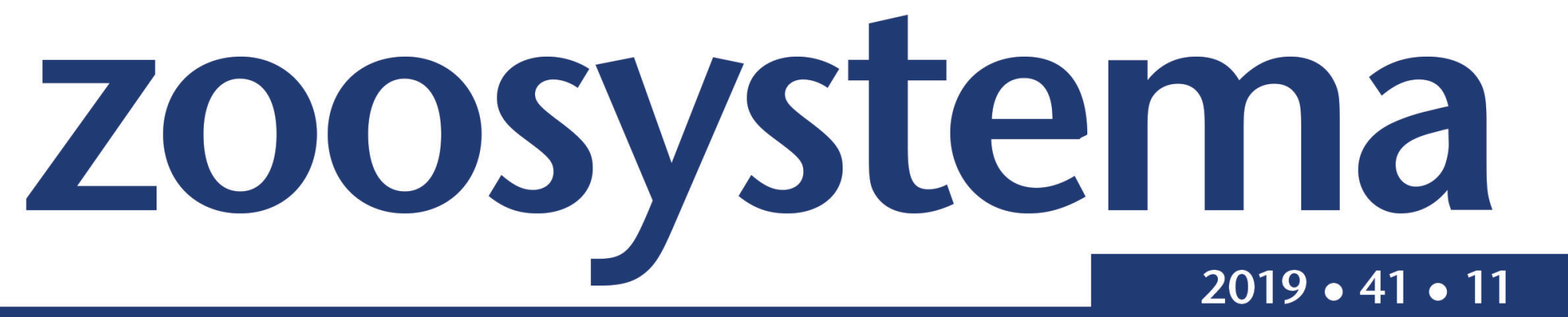

Catalogue des types de la collection de phasmes du Muséum national d'Histoire naturelle de Paris (Insecta, Phasmatodea)

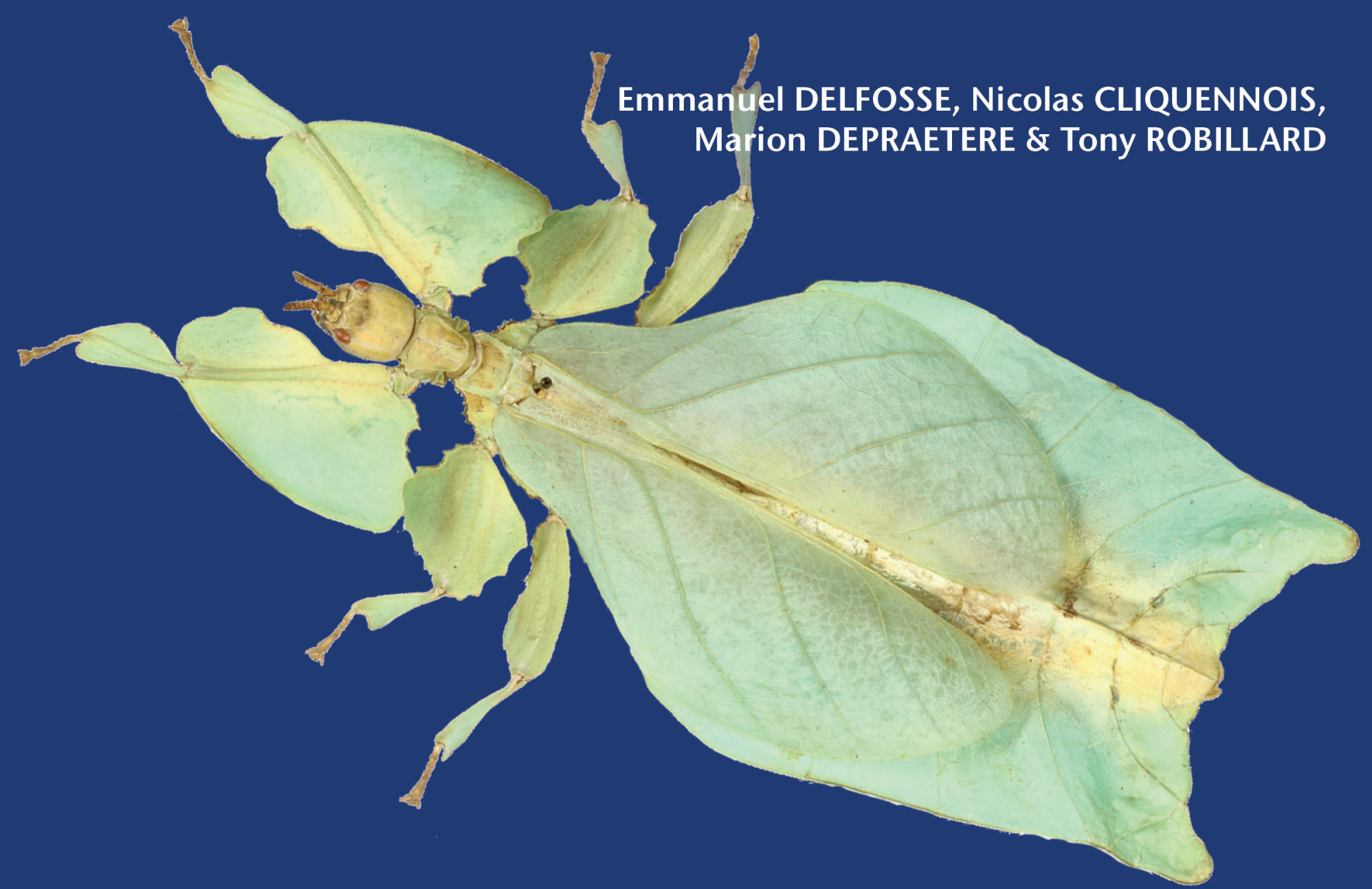

MUSÉUM 
DiRECTEUR DE LA PUBLICATION: Bruno David

Président du Muséum national d'Histoire naturelle

RÉDACTRICE EN CHEF / EDITOR-IN-CHIEF: Laure Desutter-Grandcolas

AsSISTANTS DE RÉDACTION / AsSISTANT EDITORS: Anne Mabille (zoosyst@mnhn.fr), Emmanuel Côtez

Mise en PAge / Page Layout: Anne Mabille

COMITÉ SCIENTIFIQUE / SCIENTIFIC BOARD:

James Carpenter (AMNH, New York, États-Unis)

Maria Marta Cigliano (Museo de La Plata, La Plata, Argentine)

Henrik Enghoff (NHMD, Copenhague, Danemark)

Rafael Marquez (CSIC, Madrid, Espagne)

Peter $\mathrm{Ng}$ (University of Singapore)

Norman I. Platnick (AMNH, New York, États-Unis)

Jean-Yves Rasplus (INRA, Montferrier-sur-Lez, France)

Jean-François Silvain (IRD, Gif-sur-Yvette, France)

Wanda M. Weiner (Polish Academy of Sciences, Cracovie, Pologne)

John Wenzel (The Ohio State University, Columbus, États-Unis)

COUVERTURE / COVER:

Vue dorsale de l'holotype de Phyllium maethoraniae Delfosse, 2015 (MNHN-EO-PHAS399).

Zoosystema est indexé dans / Zoosystema is indexed in:

- Science Citation Index Expanded (SciSearch $\left.{ }^{\circledR}\right)$

- ISI Alerting Services ${ }^{\circledR}$

- Current Contents ${ }^{\circledR} /$ Agriculture, Biology, and Environmental Sciences ${ }^{\circledR}$

- Scopus ${ }^{\circledR}$

Zoosystema est distribué en version électronique par / Zoosystema is distributed electronically by:

- BioOne ${ }^{\circledR}$ (http://www.bioone.org)

Les articles ainsi que les nouveautés nomenclaturales publiés dans Zoosystema sont référencés par / Articles and nomenclatural novelties published in Zoosystema are referenced by:

- ZooBank ${ }^{\circledR}$ (http://zoobank.org)

Zoosystema est une revue en flux continu publiée par les Publications scientifiques du Muséum, Paris / Zoosystema is a fast track journal published by the Museum Science Press, Paris

Les Publications scientifiques du Muséum publient aussi / The Museum Science Press also publish:

Adansonia, Geodiversitas, Anthropozoologica, European Journal of Taxonomy, Naturae, Cryptogamie sous-sections Algologie, Bryologie, Mycologie.

Diffusion - Publications scientifiques Muséum national d'Histoire naturelle

CP $41-57$ rue Cuvier F-75231 Paris cedex 05 (France)

Tél. : 33 (0)1 40794805 / Fax: 33 (0)1 40793840

diff.pub@mnhn.fr / http://sciencepress.mnhn.fr

(C) Publications scientifiques du Muséum national d'Histoire naturelle, Paris, 2019

ISSN (imprimé / print): 1280-9551/ ISSN (électronique / electronic): 1638-9387 


\title{
Catalogue des types de la collection de phasmes du Muséum national d'Histoire naturelle de Paris (Insecta, Phasmatodea)
}

\author{
Emmanuel DELFOSSE \\ Muséum national d'Histoire naturelle, Direction des collections, Entomologie, \\ case postale 50, 57 rue Cuvier, F-75231 Paris cedex 05 (France) \\ emmanuel.delfosse@mnhn.fr \\ Nicolas CLIQUENNOIS \\ Collège français Jules Verne, Lot $02 \mathrm{f} 15$ Tomboarivo, \\ B.P. 141, 110 Antsirabe (Madagascar) \\ nicolascliquennois@yahoo.fr
}

Marion DEPRAETERE

Muséum national d'Histoire naturelle, Direction des collections, Entomologie, case postale 50, 57 rue Cuvier, F-75231 Paris cedex 05 (France)

depraetere.marion@mnhn.fr

Tony ROBILLARD

Institut de Systématique, Évolution, Biodiversité (ISYEB), Muséum national d'Histoire naturelle, CNRS, Sorbonne Université, EPHE, université des Antilles, case postale 50, 57 rue Cuvier, F-75231 Paris cedex 05 (France) tony.robillard@mnhn.fr

Soumis le 9 février 2018 | Accepté le 24 septembre 2018 | Publié le 4 juin 2019

MOTS CLÉS

Catalogue, insectes,

Phasmatodea, phasmes,

types, lectotypification.
urn:Isid:zoobank.org:pub:B0245CC6-7BA9-43B6-A013-6D47CF8FD74D

Delfosse E., Cliquennois N., Depraetere M. \& Robillard T. 2019. - Catalogue des types de la collection de phasmes du Muséum national d'Histoire naturelle de Paris (Insecta, Phasmatodea). Zoosystema 41 (11): 181-235. https://doi.org/10.5252/ zoosystema2019v41a11. http://zoosystema.com/41/11

RÉSUMÉ

Cet article propose la liste commentée des 824 spécimens types de la collection de phasmes du Muséum national d'Histoire naturelle de Paris, correspondant à 319 espèces (dont 287 sont considérées comme étant actuellement valides). Le catalogue coïncide avec la numérisation et l'informatisation de la collection et est accompagné d'un document électronique illustrant l'ensemble des spécimens traités. Des lectotypes sont désignés pour six espèces (Anisacantha albogeniculata Redtenbacher, 1906; Bacteria maroniensis Chopard, 1911; Gongylopus adiposus Brunner von Wattenwyl, 1907; Gratidia nimbana Chopard, 1955 ; Parectatosoma mocquerysi Finot, 1898; Tropidoderus exiguus Redtenbacher, 1908), et Oxyartes spinulosus Redtenbacher, 1908 est rétablie en tant qu'espèce valide. 


\begin{abstract}
Catalogue of the types of the Phasmid collection of the Muséum national d'Histoire naturelle de Paris (Insecta, Phasmatodea).

This paper provides the commented list of the 824 type specimens of stick-insects of the collection of the Muséum national d'Histoire naturelle of Paris, corresponding to 319 species (including 287 currently valid species). The catalogue was made together with the digitalization and the databasing of the collection; it comes with an electronic document illustrating all of the specimens which were processed. Lectotypes are here designated for six species (Anisacantha albogeniculata Redtenbacher, 1906; Bacteria maroniensis Chopard, 1911; Gongylopus adiposus Brunner von Wattenwyl, 1907; Gratidia nimbana Chopard, 1955; Parectatosoma mocquerysi Finot, 1898 Tropidoderus exiguus Redtenbacher, 1908), and Oxyartes spinulosus Redtenbacher, 1908 is restored as a valid species.
\end{abstract}

\section{INTRODUCTION}

La collection de phasmes du Muséum national d'Histoire naturelle de Paris (MNHN) compte plus de 11000 spécimens du monde entier, notamment en provenance des régions du monde ayant connu une présence française. Cette collection est alimentée depuis le début du XXe siècle par de nombreux entomologistes, tant professionnels qu'amateurs. Elle est l'une des plus importantes au monde, et comprend une grande quantité de matériel type à la fois historique et représentatif de la diversité de cet ordre d'insectes au niveau mondial : 824 spécimens ayant le statut de syntypes (587), holotypes (142), allotypes (8), paratypes (104), lectotypes (19) et paralectotypes (166).

La majeure partie de la collection de phasmes du MNHN et du matériel type qu'elle contient s'est constituée autour du matériel étudié au début du XXe siècle par $\mathrm{C}$. Brunner von Wattenwyl (1907) et J. Redtenbacher (1906, 1908). Par la suite, d'autres auteurs, ont contribué à étoffer progressivement la collection de types par leurs travaux de taxonomie. Les principaux sont listés ci-après avec la période au cours de laquelle ils ont enrichi cette collection: J. G. Audinet-Serville, Y. Bellanger, E. Blanchard, A. D. de Boisduval, I. Bolívar, P. D. Brock, L. Chopard, N. Cliquennois, O. V. Conle, Delfosse E., M. Donskoff, A. Finot, Y. Guttiérez, F. H. Hennemann, T. Jourdan, F. Langlois, P. Lelong, P.-H. Lucas, L. Milani, M. Passamonti, R. Paulian, J. Pantel, D. E. Perez-Gelabert, V. Scali, R. Shelford, Y. Sjöstedt, B. P. Uvarov et O. Zompro.

Depuis plus d'un siècle et demi, de nombreux catalogues ont été publiés sur les phasmes du monde par divers auteurs et la majeure partie du matériel type est désormais assez bien répertoriée (Brock et al. 2018). Il demeure cependant des lacunes importantes, notamment concernant les types du MNHN, dont seule une partie, la collection Pantel, a fait l'objet d'un catalogue (Brock \& Delfosse 2005).

Le présent travail a donc pour objectif de répertorier, informatiser, numériser et illustrer l'ensemble du matériel type de la collection de phasmes du MNHN. Quand la situation le justifie, nous faisons également état de synonymies et désignons des lectotypes.

\section{MATÉRIEL ET MÉTHODES}

\section{MatéRIel ÉTUdié}

Pour mener à bien ce travail de récolement de l'ensemble des types appartenant à l'ordre des Phasmatodea présents au MNHN, nous avons étudié quatre collections: la collection générale qui est la plus importante, la collection en alcool, ainsi que deux collections historiques, la collection Finot et la collection Pantel, cette dernière ayant déjà fait l'objet d'un catalogue (Brock \& Delfosse 2005). La grande majorité des spécimens sont conservés à sec, épinglés ou sur paillettes, dans des boîtes de collections. La collection en alcool comprend quelques centaines de spécimens conservés parfois depuis plus d'un siècle dans des flûtes en verre. Certains de ces spécimens sont susceptibles d'être reconditionnés à sec suivant les besoins de conservation et d'observation.

\section{SÉLECTION ET VALIDATION TYPIQUE DES SPÉCIMENS}

Pour sélectionner les spécimens types, nous avons effectué une observation systématique de chaque boîte, flûte et bocal des différentes collections. Un numéro d'inventaire unique a été attribué à chaque spécimen identifié comme type lors de ce travail sous la forme "MNHN-EO-PHAS".

Afin de réaliser ce catalogue, nous nous sommes particulièrement appuyés sur le travail de Brock et al. (2018), dont la base de données est disponible en ligne (http://phasmida. speciesfile.org), ainsi que sur les divers catalogues et travaux cités dans la liste bibliographique. Ces travaux nous ont également permis de vérifier la validité des types, des noms scientifiques et de la taxonomie (synonymie, recombinaison, homonymie).

Nous avons aussi utilisé les registres d'entrées des spécimens en collection afin de résoudre certaines contradictions entre les étiquettes des spécimens et les indications figurant dans les descriptions originales. En effet, la date mentionnée sur certaines étiquettes rectangulaires marquées "Muséum Paris» ne correspond pas forcément à la date de collecte, mais plutôt à une date d'entrée dans les registres des collections. Sur les spécimens les plus anciens, d'autres étiquettes rondes présentent également des chiffres sous la forme d'un numéro et de la date d'entrée du spécimen dans les collections. 
Enfin, il a parfois été nécessaire de mesurer les spécimens afin de comparer les mensurations des types présumés aux mesures mentionnées dans la description originale. Ces mesures ont été réalisées à l'aide d'un pied à coulisse à montre précis à $0,01 \mathrm{~mm}$ près.

La terminologie anatomique employée s'appuie particulièrement sur les travaux de Beier $(1957,1968)$, Lelong (1995, 1996a, b), Bragg (2001) et Bradler (2009).

Les spécimens mentionnés avec un numéro d'inventaire unique dans le catalogue sont donc à la fois les types présents au MNHN, mais aussi les spécimens dits "d'intérêt", qui peuvent être des spécimens cités par les auteurs mais non types, des spécimens ayant les mêmes informations que ceux de la série typique mais n'en faisant pas partie, ou encore les spécimens de l'autre sexe lorsque la description ne fait mention que d'un seul sexe. Lorsque du matériel type est connu d'autres musées ou de collections privées, celui-ci est cité dans la section «Remarques» (cf. paragraphe "Les informations typiques au sein de ce catalogue»).

\section{BASE DE DONNÉES ET INFORMATISATION}

Après identification du matériel typique du MNHN et attribution d'un numéro d'inventaire unique à chaque spécimen, l'ensemble des informations a été incorporé dans la base de données ARTHROTER du MNHN (http://collections.mnhn.fr) qui comprend tous les groupes d'insectes et d'arachnides présents au MNHN. Cette base de données est régulièrement alimentée et enrichie grâce aux activités en lien avec les collections du Muséum. La base de données compte aujourd'hui plus de 85000 spécimens dont 1212 phasmes. Les informations indiquées sont: les informations concernant la récolte du spécimen, celles concernant l'état du spécimen, les différentes déterminations, la bibliographie associée ainsi que les différents médias liés au spécimen (photographies, enregistrements sonores, vidéos, données moléculaires, etc.).

\section{ICONOGRAPHIE}

La plupart des spécimens types cités dans ce catalogue ont été photographiés. Les autres spécimens d'intérêt cités n'ont pas été photographiés, mais les informations les concernant sont disponibles sur le site science.mnhn.fr (cf. paragraphe bases de données et informatisation). Ces photographies ont été prises à l'aide d'un boîtier numérique Canon EOS 70D équipé d'un objectif Canon EF 100 mm f/2.8L Macro IS USM. Pour augmenter la profondeur de champ des photos, nous avons utilisé le logiciel CombineZP (Hadley 2010) afin d'obtenir des images combinées à partir de plusieurs photos d'une même vue d'un spécimen. De légers ajustements de contraste et de luminosité ont été apportés à l'aide du logiciel Adobe Photoshop 12.

La numérisation des types a été réalisée selon le protocole suivant: trois prises de vues de l'habitus, en vue dorsale, ventrale et latérale, ainsi qu'un ou plusieurs clichés d'étiquettes (si des informations sont inscrites sur le verso); ou une prise de vue pour l'habitus en vue dorsale, et une ou plusieurs photographies d'étiquettes (Fig. 1).

Pour chaque taxon, nous avons choisi, lorsque c'était possible, de numériser les types primaires (holotype, lectotype) avec trois prises de vues. Pour le reste de la série typique, une seule prise de vue par spécimen a été effectuée. Dans le cas d'un type primaire unique, si la série typique le permet, un spécimen type secondaire (paralectotype, allotype ou paratype) de l'autre sexe a aussi été photographié sous les trois angles de vue. Concernant les séries de syntypes, un spécimen en bon état de chaque sexe a été photographié selon les trois angles, tandis que les syntypes restants n'ont été photographiés qu'en vue dorsale. Si le taxon n'est représenté que par un type unique, celui-ci est automatiquement photographié en trois prises de vues. Enfin, si des œufs ou certains détails sont associés à un spécimen type, ils sont également photographiés en une seule vue.

Les photographies de tous les spécimens types (vues dorsales et étiquettes) sont disponibles dans le matériel supplémentaire au format PDF (Fig. 2A). Le reste des photographies (vues latérales et ventrales) est disponible en ligne (voir paragraphe suivant).

\section{MISE À DISPOSITION DES INFORMATIONS EN LIGNE}

Les données et les images des types du MNHN présentées dans ce catalogue sont également disponibles en ligne sur le site du MNHN (science.mnhn.fr) (Fig. 2B). Sur ce site, il est possible de retrouver le spécimen en effectuant une recherche, soit par son numéro d'inventaire unique, soit par le nom d'espèce. Il est également possible de lancer des recherches plus larges par auteur, pays, récolteur, etc.

\section{Liste illustrée des 824 types de phasmes du MNHN}

Une liste illustrée des 824 types de phasmes du MNHN illustre l'ensemble des spécimens types de phasmes de la collection du Muséum national d'Histoire naturelle de Paris. Elle est accessible via le lien http://sciencepress.mnhn.fr/sites/default/ files/documents/fr/liste_phasmes_mnhn.pdf et porte le DOI: https://doi.org/10.5252/zoosystema2019v41a11_1. Cela représente 824 spécimens types et 319 espèces (dont 287 valides). Le document est organisé par ordre alphabétique des épithètes spécifiques des taxons. Toutes les photographies ont été prises par Marion Depraetere excepté pour les types de Monoiognosis spinosa Cliquennois \& Brock 2004 (MNHN-EO-PHAS1204, MNHN-EO-PHAS1205) qui ont été photographiés par Paul Brock. Pour chaque taxon dont du matériel type est présent au MNHN, la liste de l'ensemble des spécimens types associés est présentée, ainsi que les photographies des étiquettes et une photographie de chaque spécimen en vue dorsale.

\section{ABRÉVIATIONS}

Institutions

ANSP Academy of Natural Sciences, Philadelphia;

CUMZ University Museum of Zoology Insect Collection, Cambridge, Cambridge;

DEIC Deutsches Entomologisches Institut, Eberswalde;

FSCA Florida State Collection of Arthropods, Gainesville;

HNHM Hungarian Natural History Museum, Budapest (types détruits dans un incendie);

ISNB Institut royal des Sciences naturelles de Belgique, Bruxelles;

MHNG Muséum d'Histoire naturelle de la ville de Genève, Genève;

MNHN Muséum national d'Histoire naturelle, Paris;

MNMS Museo Nacional de Ciencias Naturales, Madrid; 
MNNC Museo Nacional de Historia Natural, Santiago; MSIRI Mauritius Sugar Industry Reasearch Institute, Maurice;

MSNG Museo Civico di Storial Naturale «Giacomo Doria», Florence;

MTD Staatliches Museum für Tierkunde (= SMTD), Dresde ;

NHMUK Natural History Museum (= BMNH), Londres;

NHMW Naturhistorisches Museum, Vienne, Autriche;

MHNR Muséum d'Histoire naturelle de la Réunion, Saint-Denis;

NHRS Naturhistoriska Riksmuseet, Stockholm;

NZSI National Museum Survey of India, Calcutta;

OXUM University Museum, Oxford;

PBZT Parc botanique et zoologique de Tsimbazaza, Tananarive;

SMFD Forschungsinstitut und Naturmuseum Senckenberg, Frankfort-sur-le-Main;

RBINS Royal Belgian Institute of Natural Sciences, Bruxelles;

SMNS Staatliches Museum für Naturkunde, Stuttgart;

UMB Übersee-Museum (= UMBB), Brême;

VNMN Vietnam National Museum of Nature, Hanoi ;

ZIN Zoological Institute of Russian Academy of Sciences $(=$ ZMAS = ZIP), Saint-Pétersbourg;

ZMAN Zoologisches Müseum Amsterdam, Amsterdam;

ZMB Museum für Naturkunde der Humbolt Universität $\mathrm{zu}$ Berlin (= MNHU = ZMHB);

ZMH Zoologisches Institut und Zoologisches Museum, Universität Hamburg (= ZMUH), Hambourg.

\section{Collections privées}

coll. ASPER collection de l'Association pour la Systématique des phasmes et l'Étude de leur répartition, Le Ferradou;

coll. FH collection de Frank H. Hennemann, Kaiserslautern;

coll. NC collection de Nicolas Cliquennois, Antsirabe;

coll. FL collection de Frédéric Langlois, Le Ferradou;

coll. OC collection d'Oskar Conle, Bolsterlang;

coll. OZ collection d'Oliver Zompro, Kiel;

coll. PL collections de Philippe Lelong, Le Ferradou;

coll. PB collection de Paul Brock, Hampshire;

coll. SH collection de Sylvain Hugel, Strasbourg;

coll. SM collection de S. Motala, Maurice;

coll. GEP collection du Groupe d'étude des phasmes, Paris;

coll. YB collection de Yannick Bellanger, La Ville-Jouy.

$\begin{array}{ll}\text { Types } & \\ \text { HT } & \text { holotype; } \\ \text { AT } & \text { allotype; } \\ \text { PT } & \text { paratype; } \\ \text { ST } & \text { syntype; } \\ \text { LT } & \text { lectotype; } \\ \text { PLT } & \text { paralectotype; } \\ \text { NT } & \text { néotype. }\end{array}$

FORMAT DES INFORMATIONS PRÉSENTÉES DANS LE CATALOGUE Seule une partie des informations présentes sur les étiquettes des spécimens est présentée dans ce catalogue afin de gagner en homogénéité et en lisibilité. Les informations complètes sont cependant disponibles sur le site science.mnhn.fr et dans le matériel supplémentaire en ligne. Le catalogue est organisé selon l'ordre alphaétique des épithèthes spécifiques des espèces représentées dans la collection par du matériel type. Les termes présentés entre crochets (hormis dans la ligne de titre) correspondent à des informations non présentes sur les étiquettes mais déduites de celles-ci (mots abrégés, pays manquant, toponyme moderne, etc.).
Informations sur les types en cinq points

[1] épithète spécifique. Auteur, année [nom générique original]. La ligne de titre, centrée, présente en gras l'épithète spécifique, suivie du nom de genre sous lequel l'espèce a été initialement décrite, en gras et entre crochets.

[2] Nom générique de description. Suivi de l'épithète spécifique Auteur, année de description: page. Cette ligne présente le binom original, suivi du nom de l'auteur, d'une virgule, puis de l'année et la page de description de l'espèce.

[3] Statut actuel. Nom générique actuel, épithète spécifique Auteur, année - référence de changement de nom générique. La troisième ligne de chaque entrée, sous le titre "Statut actuel», correspond au statut taxonomique actuel de l'espèce. Lorsque celle-ci a fait l'objet d'une recombinaison, d'une homonymie ou d'une synonymie, le binom accompagné du nom de l'auteur, d'une virgule, de l'année de description (éventuellement entre parenthèses (auteur, année) lorsqu'il s'agit d'une recombinaison) est suivi du tiret haut "-_" et de la référence correspondant à ce changement sous la forme " auteur année».

[4] Matériel type au MNHN. Nombre total, statut typique et sexe des spécimens, puis liste des spécimens types de la collection, en allant à la ligne après chaque spécimen ou lot de spécimens dont les informations sont similaires.

Chaque ligne présente d'abord le nombre, le sexe et le statut de chaque type, puis est indiqué entre parenthèses le numéro d'inventaire correspondant à la base de données des insectes orthoptéroïdes du MNHN, "MNHN-EO-PHAS », avec " EO » pour « Entomologie Orthoptéroïdes» et l'abréviation « PHAS» pour "collection de Phasmatodea", suivi d'un numéro unique de spécimen. À la suite de «: " sont présentées les informations mentionnées sur les étiquettes, en commençant par le pays, les autres informations concernant la collecte (localité, coordonnées GPS, altitude, habitat), le nom du récolteur, la date de récolte, les notes supplémentaires (chiffres, nycthémère, numéros de collecte) et enfin la collection d'origine des spécimens (indiquée entre crochets lorsque cette information ne figure pas sur une étiquette). Les étiquettes mentionnant les déterminations successives et statuts taxonomiques (types, n. sp., etc.) ne sont pas rapportées ici, mais le détail de ces informations est disponible sur les photographies de l'ensemble des étiquettes de chaque spécimen présenté dans le matériel supplémentaire, et également sur le site internet du MNHN avec les photos de chacun des spécimens inventoriés.

[5] Remarque(s). Ce dernier champ, facultatif, présente des informations supplémentaires éventuelles, notamment les types connus d'autres collections et les références s'y rapportant. Cette rubrique sert également à présenter les raisons qui nous ont conduits à considérer un spécimen comme un type ou non. Les désignations de lectotypes effectuées dans ce catalogue sont également expliquées dans ce champ. Un lectotype est désigné lorsque nous supposons avoir observé l'ensemble des syntypes étudiés par l'auteur, sur la base des informations présentes dans la description (nombre de syntypes, sexe, mesures, variation 

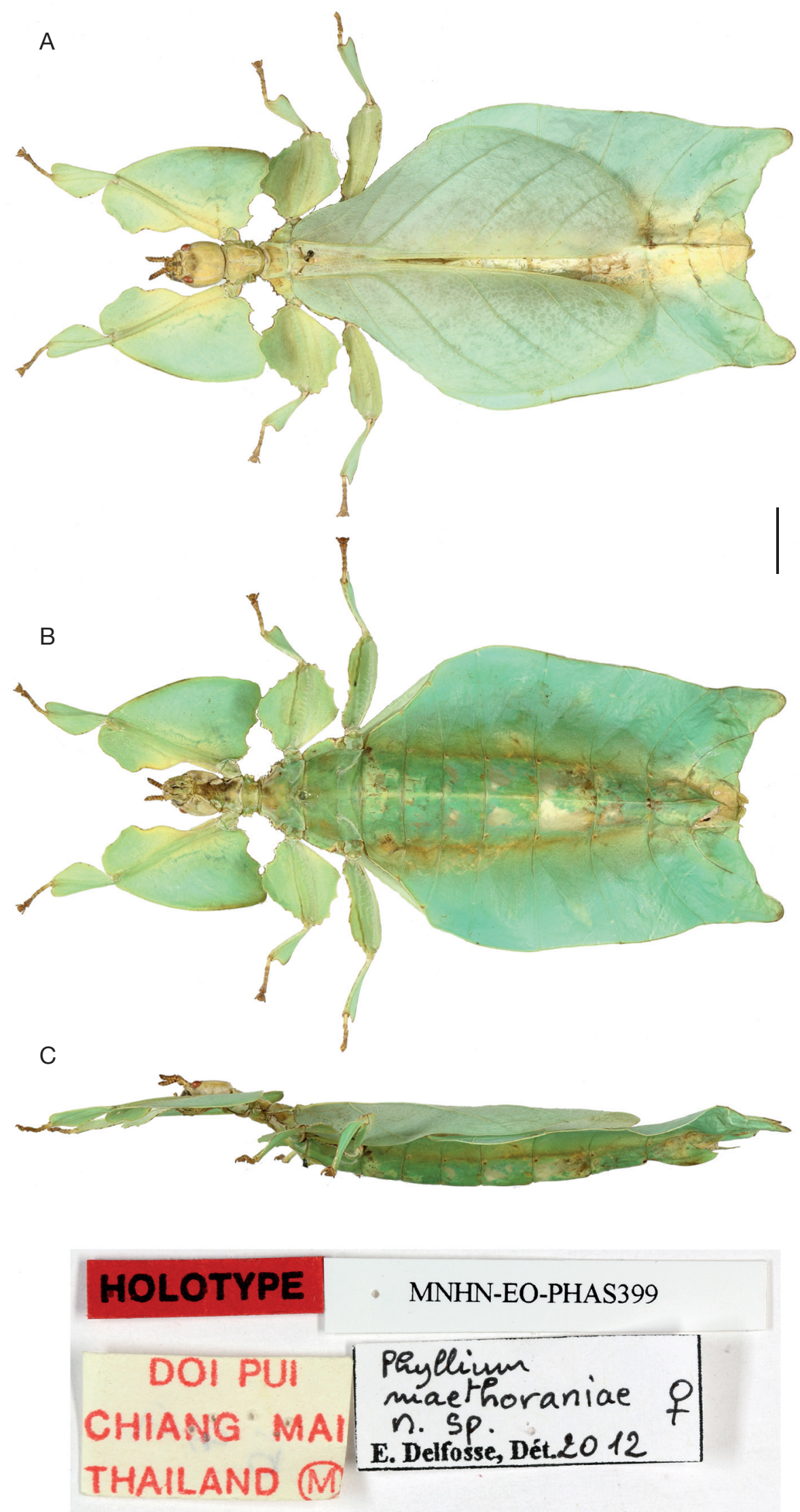

FIG. 1. - Exemple de prises de vues illustrant le catalogue disponibles sur le site science.mnhn.fr. Holotype de Phyllium maethoraniae Delfosse, 2015 (MNHNEO-PHAS399) en vue dorsale (A), ventrale (B), latérale $(\mathbf{C})$ et photo des étiquettes (D). Échelle: $1 \mathrm{~cm}$. 
A piperinus Redtenbacher, 1906 [Prisopus]

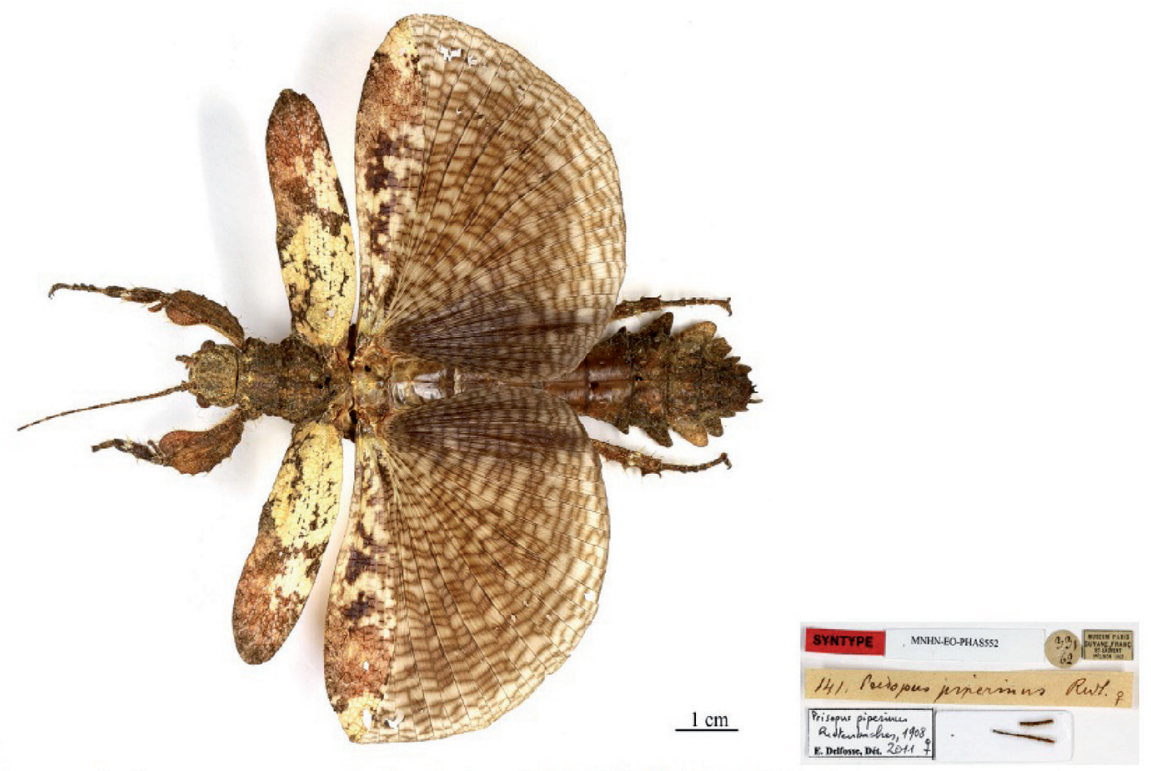

Syntype de Prisopus piperinus Redtenbacher, 1906 (MNHN-EO-PHAS552)

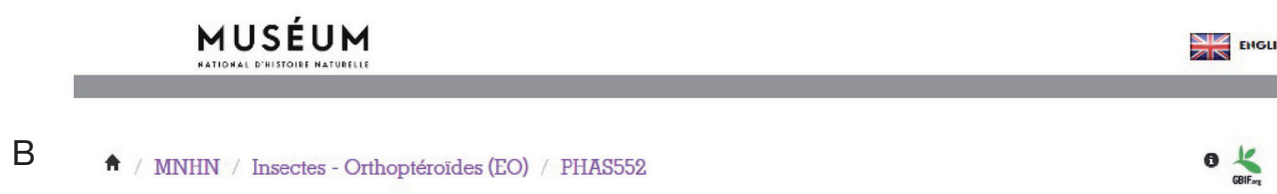

Prisopus piperinus Redtenbacher, 1906 SYNTYPE(S)
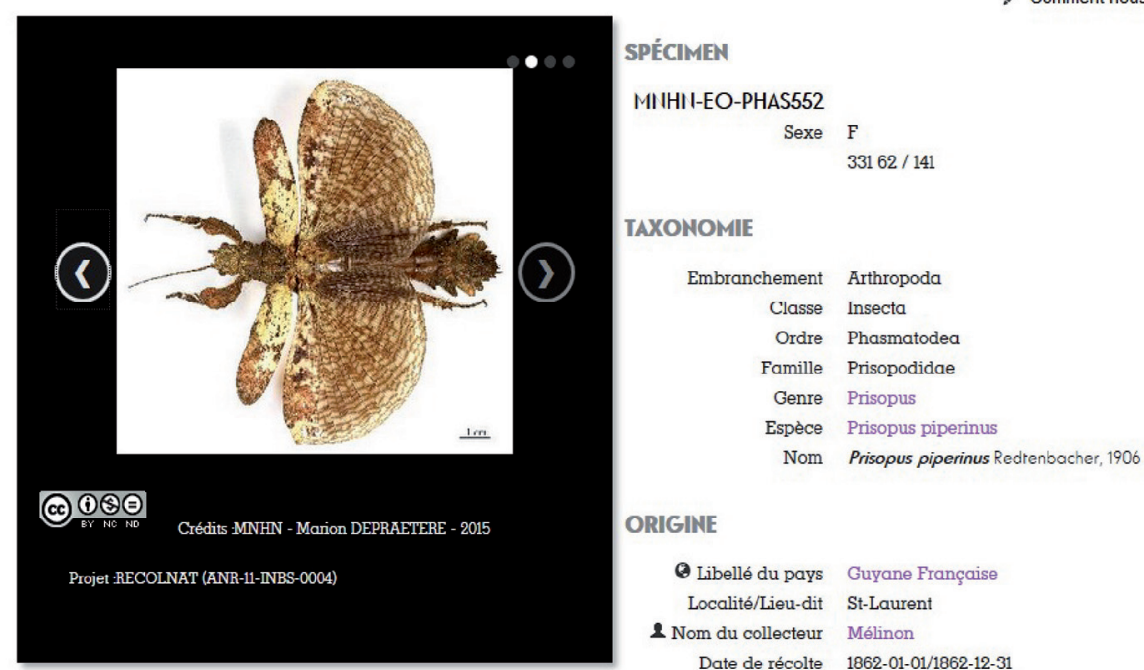

DÉTERMINATION

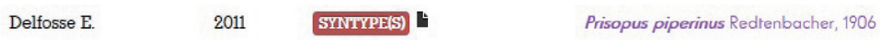


mentionnée). Le lectotype choisi correspond au spécimen de la série le plus conforme à la description. Les statuts actuels des espèces types du MNHN sont donnés selon Brock et al. (2018), avec parfois des corrections quant à la terminaison de l'épithète spécifique.

\section{LISTE DES TYPES DE PHASMES AU MNHN}

abdominalis Brunner von Wattenwyl, 1907 [Dixippus]

Dixippus abdominalis Brunner von Wattenwyl, 1907: 282.

STATUT ACTUEL. - Carausius abdominalis (Brunner von Wattenwyl, 1907) - Brock \& Delfosse 2005: 50.

MATÉRIEL TYPE AU MNHN. - HT 9.

HT $q$ (MNHN-EO-PHAS763) : [Inde], Mission Maduré, Shembaganur, [collection Pantel].

aculeata Redtenbacher, 1906 [Antongilia]

Antongilia aculeata Redtenbacher, 1906: 25.

StatUT ACtUel. - Psendonogastris aculeata (Redtenbacher, 1906) - Cliquennois 2006: 166.

MATÉRIEL TYPE AU MNHN. - $50^{7}$ ST, 5 \& ST.

5 ơ ST (MNHN-EO-PHAS937, MNHN-EO-PHAS938, MNHNEO-PHAS939, MNHN-EO-PHAS940, MNHN-EO-PHAS941), 5 O ST (MNHN-EO-PHAS942, MNHN-EO-PHAS943, MNHNEO-PHAS944, MNHN-EO-PHAS945, MNHN-EO-PHAS946) : Madagascar, Maroancetra [Maroantsetra], 12.1897, collection Finot.

\section{REMARQUES}

Les ST restants se trouvent dans trois autres musées: 3 \& , $30^{7}$ (NHMW); $10^{\prime \prime}, 2$ dont 1 juvénile probablement de l'espèce Paronogastris squamigera (Redtenbacher, 1906), une troisième o sans étiquette ayant un statut incertain $(\mathrm{ZMH}) ; 2$ ㅇ (ZIN). Redtenbacher (1906) ne mentionne que le MNHN et le NHMW comme lieu de dépôt des types, mais les spécimens du ZMH et du ZIN présentent une étiquette de détermination de la main de Brunner von Wattenwyl (Cliquennois 2003a; Zompro 2002b; Brock et al. 2018).

acuminatocercata Brunner von Wattenwyl, 1907 [Dyme]

Dyme acuminatocercata Brunner von Wattenwyl, 1907: 327.

STATUT ACTUEL. - Bacteria acuminatocercata (Brunner von Wattenwyl, 1907) - Brock et al. 2018.

MatéRIEL TYPe Au MNHN. - HT ơ.

HT ơ (MNHN-EO-PHAS985) : [Vénézuela], Llanos du Venezuela, F. Geay, 33-[18]96.

\section{adaequatum Redtenbacher, 1908 [Centema]}

Centema adaequatum Redtenbacher, 1908: 490.
STATUT ACTUEl. - Centrophasma adaequatum (Redtenbacher, 1908) - Otte \& Brock 2005: 89.

MATÉRIEL TYPE AU MNHN. - 1 ST 9 .

1 ST ๆ (MNHN-EO-PHAS986) : [Indonésie], Java, [J. D.] Pasteur, 85-[18]96.

REMARQUES

Les ST du HNHM ont été détruits dans un incendie (Otte \& Brock 2005).

\section{adefa Cliquennois, 2005 [Spathomorpha]}

Spathomorpha adefa Cliquennois, 2005: 114, figs 1-7, 10-12. STATUT ACTUEL. - Spathomorpha adefa Cliquennois, 2005.

Matériel tYPe AU MNHN. - HT o , $60^{7}$ PT, 19 ㅇ PT. HT $~(M N H N-E O-P H A S 181), 20^{7}$ PT (MNHN-EO-PHAS185, MNHN-EO-PHAS186), 3 PT (MNHN-EO-PHAS182, MNHNEO-PHAS183, MNHN-EO-PHAS184): Madagascar, province de Tamatave, Manompana, forêt d'Ambodiriana, N. Cliquennois, 100-200 m, 11-19.III.2003.

$10^{7} \mathrm{PT}$ subadulte (MNHN-EO-PHAS187), 4 ㅇ PT (MNHNEO-PHAS188, MNHN-EO-PHAS189, MNHN-EO-PHAS190, MNHN-EO-PHAS191) : Madagascar, Périnet, forêt côte Est.

1 o PT (MNHN-EO-PHAS192): [Madagascar], A. Seyrig, 1936. 1 o' PT subadulte (MNHN-EO-PHAS193), 1 ㅇ PT juvénile (MNHN-EO-PHAS194) : [Madagascar], Nanisana, A. Seyrig, XII.1932.

1 ơ PT (MNHN-EO-PHAS195) : [Madagascar], Ambodirafia, 9.II.1934.

1 ơ PT (MNHN-EO-PHAS196) : [sans étiquette].

5 ㅇ PT dont 1 juvénile (MNHN-EO-PHAS197, MNHN-EOPHAS198, MNHN-EO-PHAS199, MNHN-EO-PHAS200, MNHN-EO-PHAS201, MNHN-EO-PHAS202): Madagascar, Rogez [Andekaleka], forêt côte Est.

1 o PT juvénile (MNHN-EO-PHAS203) : Madagascar, Rogez [Andekaleka], forêt côte Est, A. Seyrig, VII.[19]32.

1 o PT (MNHN-EO-PHAS204): Madagascar, Rogez [Andekaleka], forêt côte Est, A. Seyrig, XII.1933.

1 ○ PT (MNHN-EO-PHAS205): Madagascar, Rogez [Andekaleka], forêt côte Est, Coll. A. Seyrig, XI.1932.

1 P PT (MNHN-EO-PHAS206): Madagascar, Rogez [Andekaleka], forêt côte Est, Coll. A. Seyrig, 1932.

\section{REMARQUE}

2 아 PT (PBZT) ; 1 o PT (NHMUK); 1 o PT, 2 아 PT (MHNG); $10^{7} \mathrm{PT}$ et 1 ㅇ PT (MHNL); $10^{7} \mathrm{PT}$ et 1 ㅇ PT (MHNR); 1 ơ PT et 1 ㅇ PT (Coll. PB); 1 ơ PT et 1 우 PT (Coll. FH) ; 3 ơ PT et 2 ㅇ PT (Coll. NC) (Cliquennois 2005).

adiposa Hennemann, Conle, Bellanger, Lelong \& Jourdan, 2018 [Phantasca]

Phantasca adiposa Hennemann, Conle, Bellanger, Lelong \& Jourdan, 2018: 9, figs 6-13.

Statut actuel. - Phantasca adiposa Hennemann, Conle, Bellanger, Lelong \& Jourdan, 2018.

Matériel TYPe AU MNHN. - HT 9 .

HT \& (MNHN-EO-PHAS1215): Guyane, Petit Saut, Roubaud, Auvray, Rarchaert rec., 20-31.VII.[19]92, [coll. GEP]. 
REMARQUE

1 ơ PT dans la collection OC (Hennemann et al. 2018).

\section{adiposus Brunner von Wattenwyl, 1907 [Gongylopus]}

Gongylopus adiposus Brunner von Wattenwyl, 1907: 233, pl. 9, fig. 7.

StATUT ACTUEL. — Gongylopus adiposus Brunner von Wattenwyl, 1907.

MATÉRIEL TYPe AU MNHN. - LT +1 , 9 PLT.

LT ㅇ (présente désignation) (MNHN-EO-PHAS391): [Chine],

Yunnan, Tsé-Kou, R. Oberthür, 1898, 72.

1 \& PLT (MNHN-EO-PHAS390): [Chine], Yunnan, Tsé-Kou, R. Oberthür, 1898, Nº72.

\section{REMARQUE}

Le LT a été désigné en tant que spécimen le plus proche de la description originale: «Fusco-olivaceus, nitidus. Corpus obesum. Long. Corp. ㅇ $102 \mathrm{~mm}$ (...)».

\section{adusta Redtenbacher, 1906 [Orobia]}

Orobia adusta Redtenbacher, 1906: 72.

STATUT ACTUEL. — Leiophasma adustum (Redtenbacher, 1906) Brock 1998a: 12.

MATÉRIEL TYPE AU MNHN. - 1 \% PLT.

1 o PLT (MNHN-EO-PHAS261): Madagascar, 23.

\section{REMARQUE}

LT $\sigma^{x}, 1 \sigma^{7}$ PLT et 2 ㅇ PLT au NHMW. L'appartenance des $\sigma^{7}$ et des ㅇ à la même espèce a été remise en question à plusieurs reprises (Brock 1998a; Brock et al. 2018; Cliquennois 2003a). Le LT a été désigné par Cliquennois (2003a: 11).

\section{affinis Chopard, 1911 [Isagoras]}

Isagoras affinis Chopard, 1911: 341.

STATUT ACTUEL. — Isagoras affinis Chopard, 1911.

MatérIEL TYPE AU MNHN. - $30^{7}$ ST, 5 \% ST

$20^{7}$ ST (MNHN-EO-PHAS507, MNHN-EO-PHAS508), 4 우 ST (MNHN-EO-PHAS509, MNHN-EO-PHAS510, MNHNEO-PHAS512, MNHN-EO-PHAS513): Guyane franç[aise], S[ain]t-Jean du Maroni, collection Le Moult, collection Lucien Chopard, 191.

1 ㅇ ST (MNHN-EO-PHAS511): Guyane franç[aise], Nouveau Chantier, collection Le Moult, collection Lucien Chopard, 191.

1 or ST $^{\prime M N H N-E O-P H A S 514): ~ G u y a n e ~ f r a n c ̧[a i s e], ~ S[a i n] t-~}$ Laurent du Maroni, collection Le Moult.

\section{REMARQUE}

Chopard (1911) mentionne $40^{7}$ ST et 6 ㅇ ST au total; $10^{\prime \prime}$ ST et 1 \& ST apparaissent donc manquants.

\section{albida Sjöstedt, 1909 [Gratidia]}

Gratifia albida Sjöstedt, 1909: 82, fig. 2.

Statut aCtuel. - Clonaria albida (Sjöstedt, 1909) - Otte \& Brock 2005: 98.

MATÉRIEL TYPE AU MNHN. $-10^{7}$ ST.

$10^{7}$ ST (MNHN-EO-PHAS41) : [Tanzanie], Kilimandjaro et Mérou, Meru, Nieder, Ngare na nyuki, Expédit[ion] suédoise, Y. Sjöstedt, Jan[vier], 1912.

\section{REMARQUE}

Deux autres ST ơ au NHRS (K. A. Johanson comm. pers.).

\section{albogeniculata Redtenbacher, 1906 [Anisacantha]}

Anisacantha albogeniculata Redtenbacher, 1906: 163.

STATUT ACTUEL. - Xerantherix albogeniculatus (Redtenbacher, 1906) - Zompro 2004: 245.

Matériel type au MNHN. — LT ơ 2 o $0^{7}$ PLT.

LT $\sigma^{7}$ (MNHN-EO-PHAS879) (présente désignation) : Madagascar, Sainte-Marie de Madagascar, collection Finot.

$20^{\prime \prime}$ PLT (MNHN-EO-PHAS881, MNHN-EO-PHAS880) :

Madagascar, Maroancetra [Maroantsetra], 12.1897, collection Finot.

\section{REMARQUES}

Zompro (2004) a transféré Anisacantha albogeniculata dans le genre Xerantherix Brancsik, 1893 en se fondant sur la description de Redtenbacher (1906) et sur l'examen d'un spécimen ơ de l'île de Nosy Be (ZMH), appartenant plutôt probablement à l'espèce $X$. nossibianus Brancsik, 1893, sans avoir examiné les types. L'examen des syntypes, tous $\sigma^{7}$, permet de confirmer ce transfert, mais fait apparaître que cette série typique est composée de spécimens appartenant à deux espèces. C'est pourquoi nous désignons comme LT le ơ de l'île Sainte-Marie afin d'éviter une probable synonymie, les spécimens $\sigma^{7}$ de Maroantsetra correspondant probablement à Xerantherix parvipennis Redtenbacher, 1906, espèce uniquement connue par des spécimens + et décrite à partir de spécimens également récoltés à Maroantsetra par Mocquerys en 1897. 1 ơ' PLT au NHMW (Brock 1998a; Cliquennois 2003a; Zompro 2004), non signalé par Redtenbacher (1906).

\section{albomarginatus Redtenbacher, 1906 [Damasippoides]}

Damasippoides albomarginatus Redtenbacher, 1906: 158, pl. 6, figs 1-2. STATUT ACTUEL. - Damasippoides albomarginatus Redtenbacher, 1906.

MATÉRIEL TYPE AU MNHN. - 1 \% ST.

1 ㅇ ST (MNHN-EO-PHAS909): Madagascar, collection Finot.

\section{REMARQUE}

$30^{7}$ et 5 O ST au NHMW; 1 ơ $^{7}$ et 4 o ST au ZMH; les ST du HNHM ont été détruits dans l'incendie de ce musée (Brock 1998a; Zompro 2002b; Cliquennois 2003a). 


\section{algericus Pantel, 1890 [Bacillus]}

Bacillus algericus Pantel, 1890: 394.

STATUT ACTUEL. - Clonopsis algerica (Pantel, 1890) - Pantel 1915: 96.

Matériel TYPe au MNHN. - 9 o ST, 6 9 ST, 4 juvéniles ST. $10^{7}$ ST (MNHN-EO-PHAS807), 1 ㅇ ST (MNHN-EO-PHAS808): [Algérie], Oran, 23.7.[18]80, collection Finot, [collection Pantel]. 1 o' ST (MNHN-EO-PHAS806) : [Algérie], Oran, 25.6.[18]79, collection Finot, [collection Pantel].

1 juvénile ST (MNHN-EO-PHAS1172): [Algérie], Oran, 22.3.[18]79, collection Finot.

1 juvénile ST (MNHN-EO-PHAS1173): [Algérie], Oran, 28.4. [18]79, collection Finot.

1 ơ ST (MNHN-EO-PHAS1168) : [Algérie], Oran, 16.7.[18]84, collection Finot.

2 ơ ST $^{7}$ MNHN-EO-PHAS1169, MNHN-EO-PHAS1170) : [Algérie], Oran, 4.8.[18]79, collection Finot.

1 \& ST (MNHN-EO-PHAS1174) : [Algérie], Oran, 26.8.[18]82, collection Finot.

1 \% ST (MNHN-EO-PHAS1175): [Algérie], Oran, 5.7.[18]79, collection Finot.

2 o ST (MNHN-EO-PHAS1177, MNHN-EO-PHAS1178) : [Algérie], Oran, 25.6.[18]79, collection Finot.

$40^{7}$ ST (MNHN-EO-PHAS1208, MNHN-EO-PHAS 1209 MNHN-EO-PHAS1210, MNHN-EO-PHAS1211): [Algérie], Oran, 25.6.[18]79, collection Finot.

1 ㅇ ST (MNHN-EO-PHAS1212): [Algérie], Oran, 20.6.[18]90, envoi Lemoine, collection Finot.

1 juvénile ST (MNHN-EO-PHAS1213): [Algérie], Oran, 10.6.[18]85, larva, collection Finot.

1 juvénile ST (MNHN-EO-PHAS1214): [Algérie], Oran, 28.4.[18]79, larva, collection Finot.

\section{REMARQUE}

Pantel (1890: 396) ne note pas le nombre de spécimens types, mais cite trois collections: la sienne, celle de Finot et celle de Brunner. Les spécimens de la collection Finot sont considérés ici comme des types, ainsi que d'autres dans la collection de Brunner, sans doute au NHMW aujourd'hui (Brock \& Delfosse 2005; Brock et al. 2018).

alienigenus Brunner von Wattenwyl, 1907 [Eupromachus]

Eupromachus alienigenus Brunner von Wattenwyl, 1907: 300.

STATUT ACTUEL. - Eupromachus alienigenus Brunner von Wattenwyl, 1907.

MATÉRIEL TYPE AU MNHN. - HT $~ \%$

HT \& (MNHN-EO-PHAS764) : [Inde], Mission Maduré, n³8, [collection Pantel].

\section{alligator Redtenbacher, 1908 [Canachus]}

Canachus alligator Redtenbacher, 1908: 347.

StATUT ACtUel. - Canachus alligator Redtenbacher, 1908.

MatéRIEL TYPE AU MNHN. - 5 \% ST.

1 \& ST (MNHN-EO-PHAS651): [Nouvelle-Calédonie] Léveillé, 1889, 300, [collection Pantel].

1 o ST (MNHN-EO-PHAS652): N[ouv]elle-C[alédon]ie, A. Gambey, 1891 [collection Pantel].
2 ㅇ ST (MNHN-EO-PHAS653, MNHN-EO-PHAS654) : N[ouvelle]-Calédonie, A. Gambey, 1892, [collection Pantel].

1 ST (MNHN-EO-PHAS1078): Nouv[elle]-Calédonie, "Aire du sud Bois mort», [R.] Germain, 1881, 2032 [18]81, n45.

REMARQUE

La 9 juvénile MNHN-EO-PHAS651 est notée comme un ST de Canachus alligator (Brock \& Delfosse 2005). Il s'agit cependant d'un $\sigma^{7}$ et non d'une $\%$ et très probablement d'un Canachus harpyia Redtenbacher, 1908, si nous nous appuyons notamment sur l'examen de la spinulation des pattes et du corps. Un ơ juvénile est également présent dans la collection (MNHN-EO-PHAS1073) mais n'est pas un type car seule la ○ a été décrite par Redtenbacher.

alluaudi Redtenbacher, 1906 [Pseudodatames]

Pseudodatames alluaudi Redtenbacher, 1906: 21.

Statut actuel. - Pseudodatames alluaudi Redtenbacher, 1906.

MatéRIEL TYPE AU MNHN. - HT o7.

HT Ơ (MNHN-EO-PHAS987) : Madagascar, Col de Sakavalana, Ch. Alluaud, 1901.

amabile Redtenbacher, 1906 [Paraphasma]

Paraphasma amabile Redtenbacher, 1906: 115.

StatuT ACtuel. - Paraphasma amabile Redtenbacher, 1906.

MATÉRIEL TYPE AU MNHN. $-10^{7}$ ST.

1 ơ ST (MNHN-EO-PHAS1167): Bolivie, Yungas de la Paz, 1000 m, H. Rolle, collection Finot.

\section{REMARQUE}

La collection de Paris et celle de Finot sont omises par Redtenbacher (1906). Il s'agit pourtant clairement d'un spécimen de la collection Rolle, une collection non retrouvée au NHMW (Brock 1998a). En outre la détermination est de la main même de Redtenbacher. $10^{7}$ et 1 \% ST au NHMW, 3 ơ ST et 2 우 ST au ZMUH (Brock et al. 2018).

\section{annulicornis Redtenbacher, 1906 [Antongilia]}

Antongilia annulicornis Redtenbacher, 1906: 27.

StatUT actuel. - Antongilia annulicornis Redtenbacher, 1906.

Matériel tYPe au MNHN. - HT q.

HT o juvénile (MNHN-EO-PHAS988) : [Madagascar], Nossi-Bé [Nosy Be], C. Alluaud, 1900.

REMARQUE

Le spécimen pourrait correspondre à l'espèce Canuleius delicatulus Brancsik, 1897, auquel cas l'espèce $A$. annulicornis serait un synonyme de $C$. delicatulus (Cliquennois 2006). 


\section{annulipes Redtenbacher, 1906 [Phasma]}

Phasma annulipes Redtenbacher, 1906: 119, pl. 4, fig. 16.

StATUT ACtUEl. - Pseudophasma annulipes (Redtenbacher, 1906) - Brock 1998a: 14.

MATÉRIEL TYPE AU MNHN. - 3 ơ ST, 1 \% ST.

10 ST (MNHN-EO-PHAS486) : Bolivie, Chiquitos, A. d'Orbigny, $1834,8200[18] 34,122=84$.

$10^{7}$ ST (MNHN-EO-PHAS488) : Bolivie, Chiquitos, [A. d'Orbigny, $1834,8200[18] 34,177$.

10 Ơ ST (MNHN-EO-PHAS489) : [Bolivie], Santa Cruz de la Sierra, A. d'Orbigny, 1834, 8156 [18]34.

1 ㅇ ST (MNHN-EO-PHAS487) : [Bolivie], Santa Cruz de la Sierra, A. d'Orbigny, 1834, 8156 [18]34, 84.

\section{REMARQUE}

3 ㅇ ST et 6 ơ ST au NHMW (Brock 1998a, 2007; Brock et al. 2018).

\section{arrogans Brunner von Wattenwyl, 1907 [Cuniculina]}

Cuniculina arrogans Brunner von Wattenwyl, 1907: 204.

STATUT ACTUEL. - Ramulus arrogans (Brunner von Wattenwyl, 1907) - Otte \& Brock 2005: 300.

MatéRIEL TYPE AU MNHN. - 2 \% ST.

1 ㅇ ST (MNHN-EO-PHAS707): [Inde], Kurseong, P. Décoly, [18]98, [collection Pantel].

1 o ST (MNHN-EO-PHAS708) : [Inde], Kurseong, Bengale, Kurseong, P. Décoly, [collection Pantel].

\section{arrogans Brunner von Wattenwyl, 1907 [Prosentoria]}

Prosentoria arrogans Brunner von Wattenwyl, 1907: 207, pl. 11, fig. 1a-c. STATUT ACTUEL. — Prosentoria arrogans Brunner von Wattenwyl, 1907. Matériel TYPE AU MNHN. - 1 \% ST.

1 ST (MNHN-EO-PHAS644): [Malaisie], Nord Bornéo, O. Staudinger, 1898, [collection Pantel].

\section{REMARQUE}

ST non mentionné dans la publication originale. 4 ơ ST et 3 ㅇ ST au NHMW; 1 ơ ST et 1 \% ST au MTD; 2 \% ST au ZMH; 2 ㅇ ST au ZIN (Brock 1998a, 2007; Zompro 2002b, 2003).

\section{asperrimus Redtenbacher, 1906 [Obrimus]}

Obrimus asperrimus Redtenbacher, 1906: 41, pl. 1, figs 4, 5.

STATUT ACTUEL. - Aretaon asperrimus (Redtenbacher, 1906) Bragg 1991: 76.

MATÉRIEL TYPE AU MNHN. — 4 O' ST, 3 9 ST.

$10^{7}$ ST (MNHN-EO-PHAS1197) et 1 오 ST (MNHN-EOPHAS1203): [Malaisie], Nord-Bornéo, Kina-Balu, collection Finot. $10^{7}$ ST (MNHN-EO-PHAS1200) et 1 ㅇ ST (MNHN-EOPHAS1202): Nord-Bornéo, Kina-Balu, Kinabalu, Borneo, 1500 m, H. Rolle, Berlin SW1L., collection Finot.
2 ơ ST (MNHN-EO-PHAS1198, MNHN-EO-PHAS1199) et 1 \& ST (MNHN-EO-PHAS1201) : [Bornéo], Kina-Balu, D. Vitrac dedit, collection Finot.

\section{REMARQUE}

La collection du Muséum de Paris et celle de Finot ne sont pas citées par Redtenbacher (1906). 3 o $^{7}$ ST et 3 ㅇ ST au NHMW, $40^{7}$ ST et 2 ㅇ ST au ZIN, $10^{7}$ ST et 1 ㅇ ST au MNMS (Brock 1998a, 2007; Brock et al. 2018).

\section{attenuata Pantel, 1890 [Leptynia]}

Leptynia attenuata Pantel, 1890: 402, pl. 4.

StATUT ACTUEL. - Leptynia attenuata attenuata Pantel, 1890 Scali et al. 2012: 27.

Matériel tYPe au MNHN. - LT o $0^{7}, 60^{7}$ PLT, 6 9 PLT.

LT ơ (MNHN-EO-PHAS823) : Portugal, S[an] Fiel, R. P. Baret, [18]90, [collection Pantel].

1 o PLT (MNHN-EO-PHAS825): Portugal, S[an] Fiel, P. Baret, [18]90, [collection Pantel].

1 ơ PLT (MNHN-EO-PHAS828) : [Portugal], S[an] Fiel, [18]90, [collection Pantel].

1 ơ PLT (MNHN-EO-PHAS824): Portugal, 15 articles, ongl[e] enflé « rotadet» 4, [collection Pantel].

1 ᄋ PLT (MNHN-EO-PHAS826) : [Espagne], Talavera, P. Capelle, 1889, [collection Pantel].

1 ㅇ PLT (MNHN-EO-PHAS827) et un œuf associé (MNHN-EOPHAS829): Portugal, [collection Pantel].

3 ơ PLT (MNHN-EO-PHAS1157, MNHN-EO-PHAS1158, MNHN-EO-PHAS1162): Portugal, Saint Fiel, collection Finot. $10^{7}$ PLT (MNHN-EO-PHAS1159), 2 \% PLT (MNHN-EO-PHAS1160, MNHN-EO-PHAS1161): [Espagne], Cepeda, collection Finot.

1 우 PLT (MNHN-EO-PHAS1163) et un œuf associé (MNHNEO-PHAS1164): [sans étiquette] [collection Finot].

\section{REMARQUES}

Pantel (1890) cite au moins $10^{7}$ et 1 \% de Cepeda collectés par Bolívar et $130^{7}$ du Portugal, ainsi qu'au moins un spécimen d'Urda. La seule espèce qu'Urda abrite est désormais Leptynia caprai Scali, 1996 (Scali et al. 2012). Le nombre total de types reste cependant inconnu. Certains sont de simples spécimens d'élevage comme le démontre un spécimen noté "d'élevage» et d'autres au statut incertain sont sans étiquette de provenance: $10^{7}$ (MNHN-EO-PHAS830): d'élevage, 20.VIII.98; 1 우 (MNHN-EO-PHAS835) : type ; 3 ơ (MNHN-EOPHAS831, MNHN-EO-PHAS832, MNHN-EO-PHAS833) et 3 ㅇ (MNHN-EO-PHAS834, MNHN-EO-PHAS837, MNHN-EO-PHAS838) : sans donnée. Dans la collection générale, d'autres spécimens sont trop récents ( [18]97») pour pouvoir appartenir à la série typique (MNHN-EO-PHAS1, MNHN-EO-PHAS2). Il existe trois autres o" (MNHN-EOPHAS982, MNHN-EO-PHAS983, MNHN-EO-PHAS984) du Portugal dans la boîte " mimétisme» de la collection Pantel. La o PLT de Talavera et le spécimen non retrouvé d'Urda sont très probablement des Leptynia caprai d'après leur répartition (Scali et al. 2012). Des PLT ơ et $ᄋ$ possibles au NHMW; 7 PLT $0^{7}$ et 9 au MNMS (Brock 1998a; Brock \& Delfosse 2005). Le LT a été désigné par Scali (1996: 132). 
auriculatus Redtenbacher, 1906 [Bathycharax]

Bathycharax auriculatus Redtenbacher, 1906: 31.

STATUT ACTUEL. - Bathycharax auriculatus Redtenbacher, 1908.

MatéRIEL TYPE AU MNHN. - 1 \% ST.

1 ㅇ ST (MNHN-EO-PHAS3): [Gabon] Congo, Mayumba, A. Vergnes, 1899, 136.

\section{REMARQUE}

3 ơ $^{7}$ et 1 \% ST au MHMW ; les ST du HNHM ont été détruits dans un incendie (Brock et al. 2018). La localité est actuellement située sur le territoire du Gabon.

\section{bellangeri Redtenbacher, 1908 [Bacteria]}

Bacteria bellangeri Redtenbacher, 1908: 420.

Statut aCtUel. - Paraphanocles maxwelli (Redtenbacher, 1908): 420 - Büscher \& Baker 2018: 67 (synonymie).

MatéRIEL TYPe AU MNHN. - 10 o ST.

1 ơ ST (MNHN-EO-PHAS989): Martinique, C. Bellanger, 64[18]60.

\section{REMARQUE}

1 ơ ST au NHMW (Brock 1998a).

\section{bengalensis Brunner von Wattenwyl, 1907 [Menexenus]}

Menexenus bengalensis Brunner von Wattenwyl, 1907: 246.

STATUT ACTUEL. - Spinohirasea bengalensis (Brunner von Wattenwyl, 1907) - Hennemann 2007: 20.

MATÉRIEL TYPE AU MNHN. - 1 ơ PLT, 1 \% PLT.

107 PLT (MNHN-EO-PHAS332) : Bengale, Diard \& Duvaucel, $1815,154$.

1 ㅇ PLT (MNHN-EO-PHAS331): Bengale, Cochinchine, 1815. Diard \& Duvaucel.

\section{REMARQUE}

Le LT o" est au NHMW (Brock 1998a). La mention "Bengale» apparaît douteuse. La localité "Cochinchine», soit le sud du Vietnam actuel, apparaît plus conforme à ce que l'on sait de cette espèce (Hennemann 2007). Le LT a été désigné par Brock (1998a: 17).

\section{bengalensis Redtenbacher, 1906 [Platymorpha]}

Platymorpha bengalensis Redtenbacher, 1906: 47.

STATUT ACTUEL. - Planispectrum bengalense (Redtenbacher, 1906) Bragg 1993: 44 [Planispectrum bengalensis (Redtenbacher, 1906) (sic)].

MATÉRIEL TYPE AU MNHN. - 3 9 PLT.

1 \& PLT (MNHN-EO-PHAS343) : [Indonésie], Bengkalis, M. Maindron, $1885,6983[18] 85,43$.

1 9 PLT (MNHN-EO-PHAS344): [Indonésie], Bengkalis, M. Maindron, $1885,6983[18] 85$.
1 ㅇ PLT (MNHN-EO-PHAS345) : [Indonésie], Java, J. B. Ledru, R. Oberthür, 1898.

\section{REMARQUES}

La 9 PLT (MNHN-EO-PHAS345) est l'HT de Planispectrum javanense Zompro, 2004. LT au MHMW (Brock 1998a). Le nom de genre est neutre, l'épithète spécifique doit donc être accordé en conséquence (accord rectifié). Le LT a été désigné par Brock (1998a: 17).

\section{biceps Redtenbacher, 1908 [Pharnacia]}

Pharnacia biceps Redtenbacher, 1908: 451.

StatuT ACtUel. - Tirachoidea biceps (Redtenbacher, 1908) Hennemann \& Conle 2008: 220

MATÉRIEL TYPE AU MNHN. - 10 $0^{7}$ PLT, 3 9 PLT.

10 o' PLT (MNHN-EO-PHAS401) : [Vietnam], Cho-Moï, Tonkin, M. Roget, 111- [18]96, 105.

1 PLT (MNHN-EO-PHAS990) : [Vietnam], Tonkin, Maynaud, 1768-[18]78.

1 ㅇ PLT (MNHN-EO-PHAS402) : [Indonésie], Java, Toussaint, 21-[18]62, 56.

1 ㅇ PLT (MNHN-EO-PHAS403): [Indonésie], Java, Plaboan Ratoé [Pelabuhan Ratu], R. Oberthür, 1898, 125.

REMARQUES

LT $q$ au NHMW; $40^{7}$ PLT, 4 ㅇ PLT, 4 ㅇ PLT juvéniles au NHMW ; 2 ơ PLT, 2 ㅇ PLT au MHNG ; 1 \& PLT au SMNS; 3 ㅇ PLT (dont 1 juvénile) au ZMB; 1 \& PLT au ZMH; 1 \%" PLT au ZIN; $20^{7}$ PLT et 1 \% PLT à l'ISNB (Hennemann et al. 1995; Brock 1998a, 2007; Zompro 2002b, 2006; Hennemann \& Conle 2003; Zompro \& Brock 2003). Les PLT du HNHM (nombre inconnu) ont été détruits lors d'un incendie (Hennemann et al. 1995). Hennemann \& Conle (2008) mentionnent également 3 ơ PLT, 3 o PLT et 2 juvéniles PLT au NHMW, et 2 \% PLT au ZMH. Le LT a été désigné par Hennemann \& Conle (2008: 220).

\section{bilobatus Redtenbacher, 1908 [Rhaphiderus]}

Rhaphiderus bilobatus Redtenbacher, 1908: 388.

STATUT ACTUEL. - Rhaphiderus bilobatus Redtenbacher, 1908 Cliquennois \& Brock 2004a: 56 (incertae sedis).

Matériel TYPe au MNHN. - HT o*.

HT ơ (MNHN-EO-PHAS421) : [Philippines], Manille, 246$[18] 61,111$

\section{REMARQUE}

Nec Rhaphiderus Audinet-Serville, 1838: 245 (Cliquennois \& Brock 2004a).

\section{bipunctata Cliquennois \& Brock, 2004 [Monoiognosis]}

Monoiognosis bipunctata Cliquennois \& Brock, 2004b: 6, figs 9-16, 34. 
STATUT ACtUEL. - Monoiognosis bipunctata Cliquennois \& Brock, 2004.

Matériel tyPe Au MNHN. - HT ơ, 1 ơ PT, 5 \% PT.

HT ơ (MNHN-EO-PHAS152) : Maurice, Brise Fer, Mare Longue, N. Cliquennois, 10-15.III.2001, NCMR36.

1 o PT (MNHN-EO-PHAS154): Maurice, Brise Fer, Mare Longue, 10-15.III.2001, NCMR47.

1 o PT (MNHN-EO-PHAS155): Maurice, Brise Fer, Mare Longue, 10-15.III.2001, NCMR26.

1 o PT (MNHN-EO-PHAS156): Maurice, Brise Fer, Mare Longue, 10-15.III.2001, NCMR48.

10 PT (MNHN-EO-PHAS153) : Maurice, Montagne Bambous, J. Ah-King, 15.III.2001, NCMR4.

1 ㅇ PT (MNHN-EO-PHAS157): Maurice, Les Mares, Pigeon Wood, N. Cliquennois 11.V.2002, NCMR64.

1 PT (MNHN-EO-PHAS158): [Maurice], Mauritius, Ray[mond] Mamet.

\section{REMARQUE}

2 ㅇ PT et $10^{x} \mathrm{PT}$ au NHMUK; 2 ㅇ PT et $10^{x} \mathrm{PT}$ au MHNG; $10^{7} \mathrm{PT}$ et 3 ㅇ PT (dont 1 juvénile) au MSIRI; 2 ㅇ PT et $10^{\prime \prime}$ PT au MHNR; 3 ㅇ PT et $30^{7}$ PT dans la collectionPB; 1 우 PT Coll. SH (Cliquennois \& Brock 2004b).

\section{bispinosa Chopard, 1938 [Gratidia]}

Gratidia bispinosa Chopard, 1938: 117.

STATUT ACTUEL. - Clonaria bispinosa (Chopard, 1938) - Otte \& Brock 2005: 99.

Matériel TYPe Au MNHN. - HT ơ.

HT ơ (MNHN-EO-PHAS20) : Kenya, Chip Cherangani, Marakwet, $3500 \mathrm{~m}$, Mission de l'Omo, C. Arambourg, P.-A. Chappuis \& R. Jeannel, 1932-[19]33.

\section{REMARQUE}

Un ơ subadulte (MNHN-EO-PHAS21) de la même série est mentionné par Chopard (1938) mais non cité en tant que type: Kenya, Uasin Gishu, Kitale, st. 29, 2.III.1933, Mission de l'Omo, Chip Cherangani, Marakwet, 2100 m, 1932-[19]33, C. Arambourg, P. A. Chappuis \& R. Jeannel.

\section{bispinosus Redtenbacher, 1906 [Pygirhynchus]}

Pygirhynchus bispinosus Redtenbacher, 1906: 59, pl. 1, figs 19, 20. StatUT ACtUel. - Pygirhynchus bispinosus Redtenbacher, 1906.

Matériel tYPE AU MNHN. - 1 \% ST.

1 o ST (MNHN-EO-PHAS1193): Brésil, Esperito-Santo, collection Finot.

\section{REMARQUES}

La collection de Paris et celle de Finot ne sont pas citées par Redtenbacher (1906). Il s'agit pourtant clairement d'un spécimen de la même série qu'au NHMW (Brock 1998a; Brock et al. 2018), de la collection Frühstorfer comme l'indique une de ses étiquettes, une autre présentant en outre une détermination de la main même de Redtenbacher. $10^{7}$ ST et 1 ㅇ ST au NHMW, 2 o' ST au ZMUH (Brock 1998a; Brock et al. 2018).

\section{bolivari Brunner von Wattenwyl, 1907 [Greenia]}

Greenia bolivari Brunner von Wattenwyl, 1907: 263.

StatuT aCtUel. - Carausius bolivari (Brunner von Wattenwyl, 1907) - Pantel 1917: 282.

MATÉRIEL TYPE AU MNHN. - 2 \% ST.

1 ㅇ ST (MNHN-EO-PHAS746) : [Inde], Mission Maduré, [collection Pantel].

1 \& ST en deux morceaux et ses œufs (MNHN-EO-PHAS748, MNHN-EO-PHAS750) : [Inde], Ind. Or., P. Castets, [collection Pantel].

REMARQUE

1 ㅇ (MNHN-EO-PHAS747) de la collection Pantel, sans étiquette, est de statut incertain. Plusieurs o ST au MNMS (Brock \& Delfosse 2005; Brock et al. 2018).

\section{bouvieri Redtenbacher, 1908 [Ocellata]}

Ocellata bouvieri Redtenbacher, 1908: 553.

Statut actuel. - Parasinophasma bouvieri (Redtenbacher, 1908) - Ho 2017: 182.

MATÉRIEL TYPE AU MNHN. - HT o".

HT ơ (MNHN-EO-PHAS360) : [Vietnam], Cho-Moï, Tonkin, M. Roget, 111-[18]96, 28.

\section{bouvieri Brunner von Wattenwyl, 1907 [Pachymorpha]}

Pachymorpha bouvieri Brunner von Wattenwyl, 1907: 214.

STATUT ACTUEL. - Niveaphasma annulatum (Hutton, 1898) : 162 - Jewell \& Brock 2003 [2002] : 191 (synonymie).

Matériel type AU MNHN. - 9 9 PLT. 7 \%, 2 ơ PLT.

$20^{7}$ PLT (dont 1 juvénile) (MNHN-EO-PHAS435, MNHN-EO-

PHAS436), 4 9 PLT dont 2 juvéniles (MNHN-EO-PHAS429,

MNHN-EO-PHAS430, MNHN-EO-PHAS433, MNHN-EO-

PHAS434): No[u]v[elle]-Zélande, Nelson, H. Filhol, 1876, 797 [18]76.

1 o PLT (MNHN-EO-PHAS428): No[u]v[elle]-Zélande, Nelson, H. Filhol, 1876, 797 [18]76, 185.

1 o PLT (MNHN-EO-PHAS431): No[u]v[elle]-Zélande, Nelson, H. Filhol, 1876, 797 [18]76, 48.

1 o PLT (MNHN-EO-PHAS432): No[u]v[elle]-Zélande, Nelson, H. Filhol, 1876, 797 [18]76, 51, 57=48, X gardé 1.

REMARQUE

LT $+50^{7}$ PLT et 5 \% PLT au NHMW (Brock 1998a; Brock et al. 2018). Le LT a été désigné par Brock (1998a: 19).

brevicollis Redtenbacher, 1908 [Asceles]

Asceles brevicollis Redtenbacher, 1908: 498. 
Statut actuel. - Asceles brevicollis Redtenbacher, 1908.

MATÉRIEL TYPE AU MNHN. - 1 ㅇ PLT.

1 ค PLT (MNHN-EO-PHAS377) : [Indonésie], Java Ouest, Tougou, J. D. Pasteur, 3-[18]96, 71.

\section{REMARQUE}

LT 9 au NHMW (Brock 1998a). Le LT a été désigné par Brock (1995: 87).

\section{brevipes Brunner von Wattenwyl, 1907 [Libethra]}

Libethra brevipes Brunner von Wattenwyl, 1907: 309.

StATUT ACTUEL. — Libethra brevipes Brunner von Wattenwyl, 1907.

MATÉRIEL TYPE AU MNHN. - HT 9.

HT क (MNHN-EO-PHAS600) : Mexique, S[ain]t Thomas, Antilles, A. Sallé, 60-[18]56, 60 [18]56, 3.

\section{breviuscula Bolívar, 1922 [Gratidia]}

Gratidia breviuscula Bolívar, 1922: 205.

StATUT ACTUEL. - Clonaria breviuscula (Bolívar, 1922) - Otte \& Brock 2005: 99.

MaTÉRIEL TYPE AU MNHN. - 10 ST.

1 O ST $^{7}$ STHN-EO-PHAS71): [Kenya], Afrique Orient[ale] Angl[aise], Sud du Lac Rodolphe, entre le chemin de fer et le lac, Maurice de Rothschild, 1905.

\section{brevivalvis Redtenbacher, 1906 [Orobia]}

Orobia brevivalvis Redtenbacher, 1906: 70.

STATUT ACTUEL. - Leiophasma brevivalvis (Redtenbacher, 1906) - Brock 1998a: 20.

MATÉRIEL TYPE AU MNHN. - $60^{7}$ PLT, 1 9 PLT.

$10^{7}$ PLT (MNHN-EO-PHAS259): Madagascar, 1869, 196=190.

$10^{7}$ PLT (MNHN-EO-PHAS260: Madagascar, 190.

$30^{7}$ PLT (MNHN-EO-PHAS263, MNHN-EO-PHAS264, MNHNEO-PHAS265) et 1 ㅇ PLT (MNHN-EO-PHAS266) : Madagascar. $10^{7}$ PLT (MNHN-EO-PHAS262) : Madagascar, Grandidier.

\section{REMARQUE}

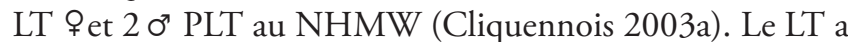
été désigné par Cliquennois (2003a: 11).

\section{brongniarti Brunner von Wattenwyl, 1906 [Clitumnus]}

Clitumnus brongniarti Brunner von Wattenwyl, 1906: 193.

STATUT ACTUEL. - Ramulus brongniarti (Brunner von Wattenwyl, 1907) - Otte \& Brock 2005: 300 (accord erroné « Ramulus brogniarti»).

MatéRIEL TYPE AU MNHN. -2 o ST.

1 ơ ST (MNHN-EO-PHAS407) : [Laos], [montagnes de] Lakhon,

J. Harmand, 1878, 990 [78], 53, 53.

$10^{7}$ ST (MNHN-EO-PHAS991): Cambodge, Harmand, 2626[18]75.
REMARQUE

Jules Harmand (1879) rapporte avoir prospecté les montagnes calcaires proches de Lakhon (actuellement Nakhon Phanom, en Thaillande), sur la rive gauche du Mékong, soit actuellement au Laos, ce qui pourrait probablement correspondre à la réserve Phou Hi Poun.

\section{brongniarti Redtenbacher, 1908 [Palophus]}

Palophus brongniarti Redtenbacher, 1908: 395.

STATUT ACTUEL. - Bactrododema episcopale (Kirby, 1896) : 466 - Brock 2004: 65 (synonymie) [Bactrododema episcopalis (Kirby, 1896) (sic)].

MatéRIel TYPE AU MNHN. - HT ơ.

HT ơ (MNHN-EO-PHAS81) : [Tanzanie], Tanganyika, Mpala, R. Oberthür, 99-[18]96.

REMARQUE

Le nom de genre est neutre, l'épithète spécifique doit donc être accordé en conséquence.

\section{calcarata Lucas, 1869 [Eurycantha]}

Eurycantha calcarata Lucas, 1869: XXV.

StATUT ACTUEL. - Eurycantha calcarata Lucas, 1869.

MATÉRIEL TYPE AU MNHN. $-20^{7}$ ST.

$20^{7}$ ST (MNHN-EO-PHAS465, MNHN-EO-PHAS466) : [Îles Salomon], Arch[ipel] Salomon, San Georges [île San Jorge], H. Lucas, 3-[18]79, 4 [18]79.

\section{REMARQUE}

Sont présentes dans les collections, des pièces génitales (MNHNEO-PHAS467) et des pièces bucales (MNHN-EO-PHAS468, MNHN-EO-PHAS469) sans attribution claire aux spécimens.

canaliculatum Audinet-Serville, 1831 [Xerosoma]

Xerosoma canaliculatum Audinet-Serville, 1831: 61.

STATUT ACTUEL. - Xerosoma canaliculatum Audinet-Serville, 1831.

MATÉRIEL TYPE AU MNHN. - HT $\%$.

HT ๆ (MNHN-EO-PHAS497) : Brésil, 135.

capitatus Brunner von Wattenwyl, 1907 [Clitumnus]

Clitumnus capitatus Brunner von Wattenwyl, 1907: 194.

STATUT ACTUEL. - Ramulus capitatus (Brunner von Wattenwyl, 1907) - Otte \& Brock 2005: 301.

Matériel TYPE AU MNHN. - HT 9.

HT \& (MNHN-EO-PHAS408) : [Vietnam], Cho-Moï, Tonkin, M. Roget, 111-[18]96, 104, 104. 
caputaurata Bellanger, Jourdan \& Lelong, 2012 [Clonistria]

Clonistria caputaurata Bellanger, Jourdan \& Lelong, 2012: 491, figs 30-37, 63-65.

STATUT ACTUEL. - Clonistria caputaurata Bellanger, Jourdan \& Lelong, 2012.

Matériel type aU MNHN. - HT $q$, AT ơ $20^{*}$ et 2 PT. HT \& (MNHN-EO-PHAS590) : [Trinité et Tobago], Mont Chaguaramal, Nord-Ouest d'Aripo, Trinidad, Yannick Bellanger, Toni Jourdan, Philippe Lelong, 07 XI 2010, TRI10-119, [Coll.] ASPER.

$10^{7}$ AT (MNHN-EO-PHAS591): [Trinité et Tobago], Station de transmission, nord d'Arima, près de Morne Bleu, Trinidad, Yannick Bellanger, Toni Jourdan, Philippe Lelong, 02.XI.2010, TRI10-024, [Coll.] ASPER.

1 \& PT (MNHN-EO-PHAS592): [Trinité et Tobago], Mont Chaguaramal, Nord-Ouest d'Aripo, Trinidad, Yannick Bellanger, Toni Jourdan, Philippe Lelong, 07 XI 2010, TRI10-122, [Coll.] ASPER. 1 ơ PT (MNHN-EO-PHAS593): [Trinité et Tobago], Trinidad, élevage ex-ovo issu de l'HT et du PT o, TRI10-138.

$10^{7}$ PT (MNHN-EO-PHAS594): [Trinité et Tobago], Trinidad, élevage ex-ovo issu de l'HT et du premier PT \& (MNHN-EOPHAS592), TRI10-137.

1 PT (MNHN-EO-PHAS595): [Trinité et Tobago], Trinidad, élevage ex-ovo issu de l'holotype et premier PT o (MNHN-EOPHAS592), TRI10-139.

carinulatum Redtenbacher, 1906 [Abrosoma]

Abrosoma carinulatum Redtenbacher, 1906: 84.

STATUT ACTUEL. - Abrosoma carinulatum Redtenbacher, 1906.

MatéRIEL TYPE AU MNHN. - 4 ơ ST.

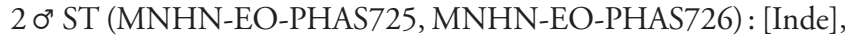
Ind[es] Or[ientales], P. Castets [collection Pantel].

1 ơ ST (MNHN-EO-PHAS722): [Inde], Mission Maduré, [collection Pantel].

1 ơ ST (MNHN-EO-PHAS723) : [Inde], Mission Maduré, 18 bis, [collection Pantel].

\section{REMARQUES}

$10^{7}$ (MNHN-EO-PHAS727) sans étiquette de la collection Pantel est un syntype potentiel. 1 \% est présente dans la collection Pantel (MNHN-EO-PHAS724) avec les informations "Mission Maduré, 18 bis» mais n'est pas un type car seul le ơ a été décrit par Redtenbacher. $10^{7}$ ST au MNMS; $10^{7}$ ST au MSNG (Brock \& Delfosse 2005; Brock et al. 2018).

\section{caryophyllea Redtenbacher, 1908 [Marmessoidea]}

Marmessoidea caryophyllea Redtenbacher, 1908: 510.

STATUT ACTUEL. - Marmessoidea vinosa (Audinet-Serville, 1838) Audinet-Serville 1838: 253 - Günther 1943: 163 (synonymie).

MATÉRIEL TYPE AU MNHN. - 3 O $^{\top}$ ST, 1 \% ST.

1 ơ ST (MNHN-EO-PHAS363) : [Indonésie], Bornéo, M. Chaper, 1891,1284 [18]91, $91=2$.

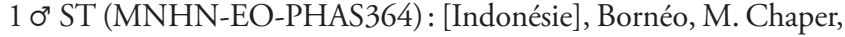
$1891,1284[18] 91,97=91$.

1 \& ST (MNHN-EO-PHAS365) : [Indonésie], Bornéo, M. Chaper,
1891,1298 [18]91, 1079.

$10^{7}$ ST (MNHN-EO-PHAS366) : [Indonésie], Bornéo, R. Oberthür, $1898,2=91$.

\section{REMARQUES}

Chaper a exploré la partie occidentale de Bornéo, autour du fleuve Kapocas (Kapuas), donc actuellement dans la partie indonésienne de l'île (Kalimatan). 7 ơ ST et 2 \% ST, 1 ST non sexé et 2 juvéniles ST (NHMW); 2 o" ST (ZMB); 2 ㅇ ST (ZIN); 1 ơ ST et 1 ㅇ ST (ZMH) (Brock 1998a; Zompro 2002b; Brock et al. 2018). Brock (2007) note 8 o $^{7}$ ST (dont 1 juvénile) et 2 9 ST au NHMW, et $10^{7}$ ST au ZMB. Vanschuytbroeck \& Cools (1981) citent 4 spécimens pour l'ISNB qui pourraient être des ST.

\section{catenulatus Redtenbacher, 1908 [Dimorphodes]}

Dimorphodes catenulatus Redtenbacher, 1908: 365.

STATUT ACTUEL. - Dimorphodes mancus catenulatus Redtenbacher, 1908. - Günther 1935a: 88.

MATÉRIEL TYPE AU MNHN. - 1 \% ST.

1 ㅇ ST (MNHN-EO-PHAS992): [Papouasie-Nouvelle-Guinée], N[ouv] elle-Guinée, Dorey [Manokwari], Raffray et Maindron, 1898.

REMARQUE

1 o' ST et 3 ㅇ ST au NHMW. Les 4 o' ST et 3 ㅇ ST au ZMB et ceux du ZMAN n'ont pas été retrouvés (Brock 1998a, b).

\section{caudatus Chopard, 1911 [Hirtaleius]}

Hirtaleius caudatus Chopard, 1911: 346, fig. [sans numéro].

STATUT aCtUel. - Hirtaleius caudatus Chopard, 1911.

MATÉRIEL TYPE AU MNHN. - HT 9 .

HT \& (MNHN-EO-PHAS543) : Guyane franç[ai]se, S[ain]t-Jean du Maroni, Août [1908], collection Le Moult, collection Lucien Chopard 1914.

\section{REMARQUE}

Une autre o (MNHN-EO-PHAS544) n'est pas répertoriée par Chopard (1911): Guyane franç[aise], S[ain]t-Laurent du Maroni, novembre, collection Le Moult.

\section{cervinum Redtenbacher, 1906 [Parectatosoma]}

Parectatosoma cervinum Redtenbacher, 1906: 166.

STATUT ACtUEl. - Parectatosoma cervinum Redtenbacher, 1906.

MATÉRIEL TYPE AU MNHN. - 1 ㅇ ST.

1 \& ST (MNHN-EO-PHAS993) : Madagascar, Col de Sakavalana, C. Alluaud, 1901.

REMARQUE

1 ST o (juvénile) au NHMW (Brock 1998a). 
chaguaramalensis Bellanger, Jourdan \& Lelong, 2012 [Apteroxylus]

Apteroxylus chaguaramalensis Bellanger, Jourdan \& Lelong, 2012: 485 , figs $1-5$.

STATUT ACTUEL. - Apteroxylus chaguaramalensis Bellanger, Jourdan \& Lelong, 2012.

Matériel tyPe aU MNHN. - HT o".

HT ơ (MNHN-EO-PHAS632) : [Trinité et Tobago], Mont Chaguaramal, nord-ouest d'Aripo, Trinidad, Bellanger, Toni Jourdan, Philippe Lelong, 07 XI 2010, TRI10-034, [Coll.] ASPER.

\section{chaperi Redtenbacher, 1908 [Bacteria $]$}

Bacteria chaperi Redtenbacher, 1908: 419.

STATUT ACTUEL. - Bacteria chaperi Redtenbacher, 1908.

MATÉRIEL TYPE AU MNHN. - HT 9 .

HT \& (MNHN-EO-PHAS574): Venezuela, M. Chaper, 1885, $1290[18] 85,46$.

\section{chaperi Redtenbacher, 1908 [Ocellata]}

Ocellata chaperi Redtenbacher, 1908: 553.

STATUT ACTUEL. - Orthonecroscia chaperi (Redtenbacher, 1908) Otte \& Brock 2005: 238.

MatéRIEL TYPe AU MNHN. - HT ơ.

HT ơ (MNHN-EO-PHAS359) : [Indonésie], Bornéo, M. Chaper, 1891.

\section{REMARQUES}

Chaper a exploré la partie occidentale de Bornéo, autour du fleuve Kapocas (Kapuas), donc actuellement dans la partie indonésienne de l'île (Kalimatan). Une ancienne étiquette placée sous ce spécimen indique "Ocellata bouvieri Br. type» mais un autre spécimen ơ (MNHN-EO-PHAS360), bien distinct, est déjà noté ainsi et provient du Vietnam. Il s'agit clairement ici d'un problème d'étiquetage. Seow-Choen (2016), après l'avoir étudié, a de fait attribué ce spécimen à $O$. chaperi.

\section{chinensis Brunner von Wattenwyl, 1907 [Cuniculina]}

Cuniculina chinensis Brunner von Wattenwyl, 1907: 202.

STATUT ACTUEL. - Baculonistria chinensis (Brunner von Wattenwyl, 1907) - Bresseel \& Constant 2014: 4.

MATÉRIEL TYPe AU MNHN. - 1 \% PLT.

1 ㅇ PLT (MNHN-EO-PHAS409) : [Chine], Kiang-si [Jiangxi], A. David, 1875, 580 [18]74, 204, 48, 48.

\section{REMARQUES}

Un spécimen ơ est présent dans la collection Pantel (MNHNEO-PHAS646) avec une étiquette marquée type, mais ni ce spécimen, ni la localité ne sont mentionnés par Brunner von Wattenwyl: [Chine], Chang Hai [Shanghai], Joanns, [18]96]. 2 ㅇ PLT au ZIN; 1 \% PLT au ZIN; LT $0^{*}, 10^{*}$ et 2 P PLT au NHMW (Brock 1998a, 2007; Brock \& Delfosse 2005; Bresseel \& Constant 2014). Le LT a été désigné par Bresseel \& Constant (2014: 4).

chinensis Brunner von Wattenwyl, 1907 [Lonchodes]

Lonchodes chinensis Brunner von Wattenwyl, 1907: 259.

Statut actuel. - Phraortes chinensis (Brunner von Wattenwyl, 1907) - Hennemann et al. 2008: 22.

MATÉRIEL TYPE AU MNHN. - $30^{7}$ ST.

10 O ST (MNHN-EO-PHAS394) : [Chine], Kiang-si [Jiangxi],

A. David, 1875, 582 [18]75, 54, 62.

1 ơ ST (MNHN-EO-PHAS395): [Chine], Kiang-si [Jiangxi],

A. David, 1869, 713 [18]69, 175, 433.

1 ơ ST (MNHN-EO-PHAS396) : [Chine], Kiang-si [Jiangxi],

A. David, 1869, 713 [18]69.

REMARQUE

2 ㅇ ST et 1 ơ ST (NHMW) (Brock 1998a).

\section{chopardi Cliquennois, 2003 [Antongilia]}

Antongilia chopardi Cliquennois, 2003a: 7.

Statut aCtUel. - Antongilia chopardi Cliquennois, 2003.

Matériel type au MNHN. -1 HT $0^{*}$.

HT ơ (MNHN-EO-PHAS310): Madagascar, M[on]t Tsaratanana, forêt de mousses, 1500-1800 m, R. P[aulian], X.[19]49, Inst. Scient. Madagascar.

REMARQUE

Antongilia quadrituberculata Chopard, 1952 a été renommé A. chopardi pour raison d'homonymie (Cliquennois 2003a).

\section{cochinchinensis Redtenbacher, 1908 [Dimorphodes]}

Dimorphodes cochinchinensis Redtenbacher, 1908: 368.

STATUT ACtUEl. - Neohirasea cochinchinensis (Redtenbacher, 1908) - Otte \& Brock 2005: 217.

Matériel TYPE AU MNHN. - HT 9 .

HT (MNHN-EO-PHAS333) : [Vietnam], Cochinchine, J. F. T. Eydoux, 119-[18]38, 119 [18]38, 150.

\section{cochinchinensis Redtenbacher, 1906 [Platymorpha]}

Platymorpha cochinchinensis Redtenbacher, 1906: 46, pl. 1, fig. 11-11a.

STATUT ACTUEL. - Planispectrum cochinchinense (Redtenbacher, 1906)- Rehn \& Rehn 1939: 484 [Planispectrum cochinchinensis (Redtenbacher, 1906) (sic)].

MATÉRIEL TYPE AU MNHN. - $10^{7}$ ST.

1 ơ ST (MNHN-EO-PHAS994) : [Vietnam], Cochinchine, $817-$ 1886. 


\section{REMARQUE}

La + ST mentionnée au MNHN par Redtenbacher (1906) n’a pas été retrouvée. Il s'agit probablement du spécimen 9 du NHMW répertorié par Brock (1998a), étiqueté "Mus. Paris ». Le nom de genre est neutre, l'épithète spécifique doit donc être accordé en conséquence (accord rectifié).

\section{concolor Redtenbacher, 1906 [Orobia]}

Orobia concolor Redtenbacher, 1906: 71

STATUT ACTUEL. - Leiophasma concolor (Redtenbacher, 1906) Brock 1998a: 22.

MATÉRIEL TYPe AU MNHN. - 10 PlT.

10 PLT (MNHN-EO-PHAS239) : [la Réunion], Bourbon, Fairmaire, 42-[18]96, 25, 25.

\section{REMARQUE}

LT ơ au MHMW (Cliquennois 2003a). La localité indiquée sur l'étiquette du PLT ("Bourbon» = La Réunion) est probablement erronée (Cliquennois 2007). Le LT a été désigné par Cliquennois (2003a: 12).

\section{contractus Brunner von Wattenwyl, 1907 [Bacunculus]}

Bacunculus contractus Brunner von Wattenwyl, 1907: 335.

STATUT ACTUEL. - Heteronemia contracta (Brunner von Wattenwyl, 1907) - Otte \& Brock 2005: 158.

Matériel TYPe AU MNHN. - 1 O ST, 1 \% ST.

$10^{7}$ et 1 (juvénile) ST (MNHN-EO-PHAS995, MNHN-EOPHAS996): Mexique, État Jalisco, L. Diguet, 1900.

\section{contractus Brunner von Wattenwyl, 1907 [Lonchodes]}

Lonchodes contractus Brunner von Wattenwyl, 1907: 260.

STATUT ACTUEL. - Menexenus semiarmatus (Westwood, 1848) : 15, pl. 7, fig. 2 - Günther 1938: 127 (synonymie).

Matériel TYPE AU MNHN. - HT ơ.

HT ơ (MNHN-EO-PHAS656) : [Inde], Kurseong, P. Décoly, [18]98, [collection Pantel].

\section{coriacea Redtenbacher, 1906 [Xylica $]$}

Xylica coriacea Redtenbacher, 1906: 29.

StatuT ACtUel. - Xylica coriacea Redtenbacher, 1906.

Matériel TyPe au MNHN. - HT ơ.

HT ơ (MNHN-EO-PHAS4) : [Gabon], Bas-Ogooué, entre Lambaréné et la mer, E. Haug, 1901.

corticina Redtenbacher, 1906 [Peripbloea]

Periphloea corticina Redtenbacher, 1906: 152.
STATUT ACtUel. - Periphloea corticina Redtenbacher, 1906.

MATÉRIEL TYPE AU MNHN. - 1 \% ST.

1 S ST (MNHN-EO-PHAS519): Guyane franç[aise], Maroni, $20 \mathrm{~km}$ aval du saut Hermina, H. Lourtau, 1901.

REMARQUE

Zompro (2004a) mentionne 2 \& ST, mais un seul spécimen type a pu être retrouvé.

corticinus Redtenbacher, 1906 [Dares]

Dares corticinus Redtenbacher, 1906: 56.

STATUT ACTUEL. - Dares ulula (Westwood, 1859)- Westwood 1859: 53, pl. 26, fig. 1. - Günther 1935b: 3 (synonymie).

MATÉRIEL TYPE AU MNHN. - HT o".

HT ơ (MNHN-EO-PHAS339) (juvénile) : [Indonésie], Bornéo, M. Chaper, 1891, 1281 [18]91, 33.

REMARQUE

Chaper a exploré la partie occidentale de Bornéo, autour du fleuve Kapocas (Kapuas), donc actuellement dans la partie indonésienne de l'île (Kalimatan).

crassa Blanchard, 1851 [Anisomorpha]

Anisomorpha crassa Blanchard, 1851a, b: 28, pl. 1, fig. 7.

StatUT aCtUel. - Agathemera crassa (Blanchard, 1851) - Berg 1899: 183.

Matériel tYPe AU MNHN. - LT 9 , 1 ㅇ PLT.

LT \& (MNHN-EO-PHAS560) : Chili, C. Gay, 15-[18]43, 15 [18]43, 163, 163, 2 ex.

1 ㅇ PLT (MNHN-EO-PHAS561) : Chili, Coquimbo, C. Gay, 15[18]43, 15 [18]43, 92.

REMARQUE

Le ơ étudié par Blanchard (1851a, b) n’a pas été retrouvé. La + PLT correspond à l'espèce Agathemera maculafulgens Camousseight, 1995 (Camousseight 1995). Le LT a été désigné par Camousseight (1995: 41).

crassipes Redtenbacher, 1908 [Asprenas]

Asprenas crassipes Redtenbacher, 1908: 349.

Statut aCtuel. - Asprenas crassipes Redtenbacher, 1908.

MATÉRIEL TYPE AU MNHN. - HT 9.

HT 9 (MNHN-EO-PHAS997) : N[ouv]elles-Hébrides [Vanuatu], Canal de Péjard, Dr François, 786-[18]92.

crassipes Chopard, 1911 [Bacteria]

Bacteria crassipes Chopard, 1911: 344, fig. [sans numéro]. STATUT ACtUel. - Bacteria crassipes Chopard, 1911. 
MATÉRIEL TYPE AU MNHN. - HT 9

HT \& juvénile (MNHN-EO-PHAS564): Guyane franç[ai]se, S[ain]t-Laurent du Maroni, collection Le Moult, collection Lucien Chopard, 191.

crudis Brunner von Wattenwyl, 1907 [Ocnophila]

Ocnophila crudis Brunner von Wattenwyl, 1907: 312.

STATUT ACTUEL. - Pseudosermyle phalangiphora (Rehn, 1907) Rehn 1907: 229 - Conle et al. 2007: 38 (synonymie).

MATÉRIEL TYPE AU MNHN. - HT 9.

HT (MNHN-EO-PHAS631) : Mexique, A. Sallé, 97-[18]56, $97[18] 56,24$.

cuspidata Redtenbacher, 1906 [Hoploclonia]

Hoploclonia cuspidata Redtenbacher, 1906: 46.

STATUT ACTUEL. - Hoploclonia cuspidata cuspidata Redtenbacher, 1906 - Seow-Choen 2016: 416.

MATÉRIEL TYPE AU MNHN. - HT 9 .

HT 9 (MNHN-EO-PHAS998): [Malaisie], Nord Bornéo, J. Whitehead 918-[18]83.

\section{cylindriceps Brunner von Wattenwyl, 1907 [Clitumnus]}

Clitumnus cylindriceps Brunner von Wattenwyl, 1907: 190.

STATUT ACTUEL. - Ramulus cylindriceps (Brunner von Wattenwyl, 1907) - Otte \& Brock 2005: 301.

MatéRIEL TYPE AU MNHN. - $30^{\circ}$ ST.

1 ơ ST (MNHN-EO-PHAS728): [Inde], Kurseong, Décoly, [18]98, 76, [collection Pantel].

1 ơ ST (MNHN-EO-PHAS729) : [Inde], Kurseong, Bengale, [collection Pantel].

$10^{7}$ ST (MNHN-EO-PHAS730): [Inde], Mission Maduré, 76, [collection Pantel].

\section{cylindripes Redtenbacher, 1906 [Datames]}

Datames cylindripes Redtenbacher, 1906: 51.

StatuT ACtUEL. - Pylaemenes oileus (Westwood, 1859) - Westwood 1859: 53, pl. 26, fig. 4 - Günther 1935a [1934]: 76 (synonymie); Brock 1996: 15, fig. (nouvelle combinaison).

Matériel tYPe aU MNHN. — LT 9 , 19 PLT.

LT \& (MNHN-EO-PHAS341) : [Indonésie], Java, Tjibogo-Preanger [Parahyangan], J. B. Ledru, R. Oberthür, 1898, 75, 75.

1 P PLT (MNHN-EO-PHAS1196) : [Indonésie], Java, collection Finot.

\section{damicornis Bolívar, 1922 [Gratidia]}

Gratidia damicornis Bolívar, 1922: 207.

STATUT ACTUEL. - Clonaria damicornis (Bolívar, 1922) - Otte \& Brock 2005: 100
Matériel TYPE AU MNHN. - HT ơ.

HT ơ (MNHN-EO-PHAS78): [Kenya], Afrique Orient[ale] Angl[aise], Sud du Lac Rodolphe, entre le chemin de fer et le lac, Maurice de Rothchild, 1905.

debilis Brunner von Wattenwyl, 1907 [Carausius]

Carausius debilis Brunner von Wattenwyl, 1907: 270.

STATUT ACtUEL. — Carausius debilis Brunner von Wattenwyl, 1907.

MATÉRIEL TYPE AU MNHN. - 3 9 ST.

1 ㅇ ST (MNHN-EO-PHAS740): [Inde], Mission Maduré, [collection Pantel].

1 o ST (MNHN-EO-PHAS741) : [Inde], Kodikanel [Kodaikanal], Décoly, [collection Pantel].

1 ST (MNHN-EO-PHAS742): $\mathrm{n}^{\circ} 25$, [collection Pantel].

REMARQUE

D'autres o ST possibles au MNMS (Brock \& Delfosse 2005). Les spécimens du MNHN constituent probablement un don de Bolívar (dont la collection est la seule à être mentionnée comme lieu de dépôt par Brunner von Wattenwyl) à Pantel.

\section{decolyanus Brunner von Wattenwyl, 1907 [Lonchodes]}

Lonchodes decolyanus Brunner von Wattenwyl, 1907: 260.

STATUT ACtUel. - Lonchodes decolyanus Brunner von Wattenwyl, 1907.

MATÉRIEL TYPE AU MNHN. - $30^{7}$ ST, 2 9 ST.

2 o7 ST (MNHN-EO-PHAS657, MNHN-EO-PHAS659), 1 \% ST (MNHN-EO-PHAS658) : [Inde], Kurseong, Décoly, X.[18]99, [collection Pantel].

1 ơ ST (MNHN-EO-PHAS660): [Inde], Mission Maduré, Kurseong, Décoly, X.[18]99, [collection Pantel].

1 o ST (MNHN-EO-PHAS661) : [Inde], Kurseong, Bengale, [collection Pantel].

REMARQUE

2 ơ ST au NHMW : Kurseong, ex coll. Pantel, 496 (Brock 1998a; Brock \& Delfosse 2005).

\section{decolyi Brunner von Wattenwyl, 1907 [Cuniculina]}

Cuniculina decolyi Brunner von Wattenwyl, 1907: 202.

STATUT ACTUEL. - Ramulus decolyi (Brunner von Wattenwyl, 1907) - Otte \& Brock 2005: 301

MatérIel TYPE AU MNHN. - 1 \% ST.

1 ㅇ ST (MNHN-EO-PHAS709) : [Inde], Kurseong, Décoly, 1899, «les. œuf» 77, [collection Pantel].

REMARQUE

1 ST au NHMW (Brock \& Delfosse 2005). 
denticulata Redtenbacher, 1908 [Graeffea]

Graeffea denticulata Redtenbacher, 1908: 371.

STATUT ACTUEL. - Acanthograeffea denticulata (Redtenbacher, 1908) - Günther 1932: 776.

MATÉRIEL TYPE AU MNHN. -2 o ST, 11 \% ST.

1 ơ ST (MNHN-EO-PHAS999), 6 \% ST dont 2 juvéniles (MNHNEO-PHAS1001, MNHN-EO-PHAS1002, MNHN-EO-PHAS1006, MNHN-EO-PHAS1007, MNHN-EO-PHAS1008, MNHN-EOPHAS1009) : [États-Unis], Mariannes, A. Marche, 169-[18]89.

$10^{7}$ ST (MNHN-EO-PHAS1000), 5 o dont 2 subadultes ST (MNHN-EO-PHAS1003, MNHN-EO-PHAS1004, MNHN-EOPHAS1005, MNHN-EO-PHAS1136, MNHN-EO-PHAS1137).

\section{REMARQUE}

4 ㅇ ST au NHMW (Brock 1998a). 6 œufs issus du matériel type (MNHN-EO-PHAS1070).

\section{descampsi Conle, Hennemann \& Guttiérez, 2011 [Atratomorpha]}

Atratomorpha descampsi Conle, Hennemann \& Guttiérez, 2011: 101.

STATUT ACTUEL. - Atratomorpha descampsi Conle, Hennemann \& Guttiérez, 2011.

Matériel tYPe Au MNHN. - HT ơ, 1 9 PT.

HT o" (MNHN-EO-PHAS494) : Colombie, Dept. Putumayo, entre El Mirador et Pepino, 1500 m, M. Descamps, 8-XI-1968.

1 ㅇ PT (MNHN-EO-PHAS495): Colombie, Dept. Putumayo, San Francisco, 2500 m, M. Descamps, 7-XI-1968.

\section{descendens Redtenbacher, 1908 [Parasosibia]}

Parasosibia descendens Redtenbacher, 1908: 482.

StATUt ACtUel. - Parasosibia descendens Redtenbacher, 1908.

MATÉRIEL TYPE AU MNHN. - 1 \% ST.

1 o ST (MNHN-EO-PHAS796): [Inde], Mission Maduré, [collection Pantel].

\section{REMARQUE}

1 (MNHN-EO-PHAS797) de la collection Pantel, sans étiquette, est de statut incertain. D'autres ST présents au MNMS (Brock \& Delfosse 2005).

\section{diabolus Redtenbacher, 1908 [Eurycantha]}

Eurycantha diabolus Redtenbacher, 1908: 343.

STATUT ACTUEL. - Eurycantha calcarata Lucas, 1869: 25 - Günther 1929: 621 (synonymie).

MatéRIel TYPe AU MNHN. - 1 \% ST.

1 o ST (MNHN-EO-PHAS471) : [Australie], N[ouvelle]-Hollande,

D. d'Urville, 153.
REMARQUE

D’après Zompro (2005), l'Australie serait répertoriée par erreur car tous les autres spécimens proviennent de diverses localités situées en Nouvelle-Guinée. $10^{7}$ ST au NHMW; 1 o' ST au ZMH; 2 o ST au ZMB (Brock, 1998a). Zompro (2002) signale $10^{\text {x ST }}$ et 2 ㅇ ST au ZMH.

\section{dicranocollis Redtenbacher, 1906 [Leprodes]}

Leprodes dicranocollis Redtenbacher, 1906: 23, pl. 2, fig. 9.

STATUT ACTUEL. - Leprodes dicranocollis Redtenbacher, 1906.

MatéRIEL TYPE AU MNHN. - 1 \% PLT.

1 ㅇ PLT (MNHN-EO-PHAS1010): Madagascar, Isaka, Ch. Alluaud, 1901.

REMARQUE

LT + au NHMW (Brock 1998a; Zompro 2002a). Le LT a été désigné par Zompro (2002a: 6).

\section{differens Redtenbacher, 1908 [Hermarchus]}

Hermarchus differens Redtenbacher, 1908: 445.

STATUT ACTUEL. - Hermarchus differens Redtenbacher, 1908.

MATÉRIEL TYPE AU MNHN. - HT 9.

HT (MNHN-EO-PHAS453) : [Fidji], I[le] Viti, Lebouka [Levuka], H. Jacquinot, 1841, 65.

\section{difformis Redtenbacher, 1906 [Anisacantha]}

Anisacantha difformis Redtenbacher, 1906: 163, pl. 6, figs 6, 7. STATUT ACTUEL. - Anisacantha difformis Redtenbacher, 1906.

MATÉRIEL TYPE AU MNHN. - $40^{7}$ et 10 오 ST.

$40^{7}$ ST (MNHN-EO-PHAS470, MNHN-EO-PHAS579, MNHN-EO-PHAS867, MNHN-EO-PHAS868) et 10 \% ST dont 5 juvéniles (MNHN-EO-PHAS866, MNHN-EO-PHAS869, MNHN-EO-PHAS871, MNHN-EO-PHAS872, MNHN-EOPHAS873, MNHN-EO-PHAS874, MNHN-EO-PHAS875, MNHN-EO-PHAS876, MNHN-EO-PHAS877, MNHN-EOPHAS878) : Madagascar, Maroancetra [Maroantsetra], 12.1897, collection Finot.

\section{REMARQUES}

2 o' ST et 2 ㅇ ST au ZMH; 2 o' ST (dont 1 ơ Xerantherix sp.) et 3 ㅇ ST au ZIN (Brock 1998a, 2007; Brock et al. 2018; Zompro 2002b). Cliquennois (2003a) note aussi 11 ㅇ ST et 15 o' ST au NHMW (des adultes et des juvéniles des deux sexes), tandis que Brock (1998a) mentionne $140^{\top}$ et 12 \% . Le MHNG possède $2 \sigma^{\top}$ et $2 \%$ avec une étiquette de détermination de Brunner von Wattenwyl et qui constituent potentiellement des ST. Les $\subsetneq$ sont également des PLT de Parectatosoma mocquerysi Finot, 1898 (voir ci-après). 


\section{digueti Chopard, 1919 [Bacunculus]}

Bacunculus digueti Chopard, 1919: 153.

STATUT ACTUEL. - Pseudosermyle tridens (Burmeister, 1838) : 567

- Hebard 1932: 216 (synonymie).

MatéRIEL TYPE AU MNHN. - HT ơ.

HT ơ (MNHN-EO-PHAS598): Mexique, État de Puebla, Env[irons] de Tehuacan, L. Diguet, 1903.

\section{discolor Redtenbacher, 1908 [Diura]}

Diura discolor Redtenbacher, 1908: 382.

StatUT ACTUEL. - Didymuria violescens (Leach, 1814) - Leach 1814: 26, pl. 9 - Vickery 1983: 6 (synonymie).

Matériel TYPE AU MNHN. - $60^{7}$ ST, 5 \% ST.

$10^{7}$ ST (MNHN-EO-PHAS441) : [Australie], Tasmanie, Verreaux, 4-[18]46, 4 [18]46, 338, 109.

1 ơ ST (MNHN-EO-PHAS442), 1 ᄋ ST (MNHN-EO-PHAS443): [Australie], Tasmanie, J. Verreaux, 4-[18]46, 3 [18]47.

1 ơ ST (MNHN-EO-PHAS444), 2 \& ST (MNHN-EO-PHAS445, MNHN-EO-PHAS446): [Australie], Tasmanie, J. Verreaux, 4-[18]46, 4 [18]46.

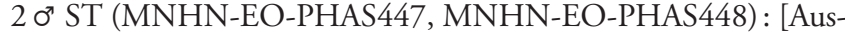
tralie], Tasmanie, J. Verreaux, 4-[18]46.

$10^{7}$ ST (MNHN-EO-PHAS1011) et 2 o ST (MNHN-EOPHAS1012, MNHN-EO-PHAS1013): Australie, Rio Munaige, J. Verreaux.

\section{REMARQUE}

2 ST et 2 ơ ST au NHMW; 2 ㅇ ST au SMNS; d'autres ST à l'UMB (Hennemann et al. 1995; Hennemann \& Conle 2003; Zompro 2006; Brock 1998a; Brock et al. 2018).

\section{distincta Brunner von Wattenwyl, 1907 [Pachymorpha]}

Pachymorpha distincta Brunner von Wattenwyl, 1907: 216.

Statut actuel. - Paracirsia distincta (Brunner von Wattenwyl, 1907) - Cliquennois 2006: 167.

Matériel TYPe AU MNHN. — LT 9 , $10^{7}$ PLT.

LT क (MNHN-EO-PHAS105), 1 o PLT (MNHN-EO-PHAS104): Madagascar, Région de l'Androy, Ambovombe, Dr J. Decorse, mars 01,1901 .

\section{REMARQUE}

Le LT a été désigné par Cliquennois (2003a: 7).

\section{distinctissima Redtenbacher, 1908 [Sipyloidea]}

Sipyloidea distinctissima Redtenbacher, 1908: 546.

STATUT ACtUEL. — Sipyloidea distinctissima Redtenbacher, 1908.

Matériel TYPe AU MNHN. - HT ơ.

HT ơ (MNHN-EO-PHAS355): [Vietnam], Cochinchine, Deyrolle, 1861, 226 [18]61, 103.

\section{divergens Redtenbacher, 1908 [Bacteria]}

Bacteria divergens Redtenbacher, 1908: 418.

Statut actuel. - Phanocloidea muricata (Burmeister, 1838): Burmeister 1838: 564. - Zompro \& Brock 2003: 11 (synonymie).

MATÉRIEL TYPE AU MNHN. -1 \& ST.

1 \& ST (MNHN-EO-PHAS1014): Guyane, Ouanary, F. Geay, 1900.

\section{REMARQUE}

Seul Cayenne est cité par Redtenbacher (1908). 1 ST au MHNG (Zompro \& Brock 2003).

\section{diversus Brunner von Wattenwyl, 1907 [Clitumnus]}

Clitumnus diversus Brunner von Wattenwyl, 1907: 194.

STATUT ACTUEL. - Ramulus diversus (Brunner von Wattenwyl, 1907) - Otte \& Brock 2005: 301.

Matériel TYPe AU MNHN. - HT $q$.

HT \& (MNHN-EO-PHAS1015): [Vietnam], Tonkin, Hon-Gay [Ha Long], 1901.

\section{donskoffi Langlois \& Lelong, 1998 [Pseudobacteria]}

Pseudobacteria donskoff Langlois \& Lelong, 1998: 248, figs 10-21, 28-30.

StatUT ACTUEL. - Bacteria donskoff (Langlois \& Lelong, 1998) - Zompro 2001: 204.

MATÉRIEL TYPE AU MNHN. - HT + , AT ơ, 1 \& PT, $10^{7}$ PT.

HT \& (MNHN-EO-PHAS584): Guadeloupe, Pigeon, Chemin Poirier, Alt. 30 m, 21 XI 1996, GUA96026, [Coll. ASPER].

AT o" (MNHN-EO-PHAS585): [Guadeloupe], Pigeon, Chemin

Poirier, Alt. 20-40 m, 26 X [19]95, Frédéric Langlois, Philippe Lelong, Esp. N³, GUA95076, [Coll. ASPER].

1 ㅇ PT (MNHN-EO-PHAS586) : [Guadeloupe], Pigeon, Chemin Poirier, Alt. 20-40 m, 26 X [19]95, Frédéric Langlois, Philippe Lelong, Esp. N³, GUA95063, [Coll. ASPER].

$10^{7}$ PT (MNHN-EO-PHAS587) : [Guadeloupe], Pigeon, Chemin Poirier, Alt. 20-40 m, 26 X [19]95, Frédéric Langlois, Philippe Lelong, Esp. N³, GUA95064, [Coll. ASPER].

REMARQUE

$10^{\prime \prime}$ et 1 PT dans la Coll. PL (Lelong \& Langlois 1998; Zompro 2001).

\section{dorbignyana Brunner von Wattenwyl, 1907 [Calynda]}

Calynda dorbignyana Brunner von Wattenwyl, 1907: 329, pl. 14, fig. 4. STATUT ACTUEL. - Calynda dorbignyana Brunner von Wattenwyl, 1907.

Matériel type au MNHN. - HT ㅇ.

HT (MNHN-EO-PHAS570) : [Cuba], Santa Cruz, Valle Grande, A. d'Orbigny, 1834, 9160 [18]34, 137, 187. 


\section{dreyfusi Brunner von Wattenwyl, 1907 [Dyme]}

Dyme dreyfusi Brunner von Wattenwyl, 1907: 324.

STATUT ACTUEL. - Bacteria dreyfusi (Brunner von Wattenwyl, 1907) - Otte \& Brock 2005: 63.

Matériel type au MNHN. - HT o".

HT ơ (MNHN-EO-PHAS568): Guyane franç[aise], Mana, De La Mana, Leschen[ault], Leschenault, 195.

\section{REMARQUE}

Brunner von Wattenwyl (1907) indique «Cayenne», pourtant la description, les caractéristiques et les mesures du spécimen observé semblent bien correspondre, comme par exemple l'absence des pattes antérieures et postérieures.

\section{dubiosus Redtenbacher, 1906 [Pseudoleosthenes]}

Pseudoleosthenes dubiosus Redtenbacher, 1906: 159.

StatUT ACtUel. - Pseudoleosthenes dubiosus Redtenbacher, 1906.

MatéRIEL TYPE AU MNHN. - 1 \% ST.

1 ㅇ ST (MNHN-EO-PHAS865): Madagascar, Maroancetra

[Maroantsetra], 12.1897, collection Finot.

REMARQUE

1 ㅇ ST au NHMW (Brock 1998a).

\section{elaboratus Brunner von Wattenwyl, 1907 [Clitumnus]}

Clitumnus elaboratus Brunner von Wattenwyl, 1907: 195.

STATUT ACTUEL. - Ramulus elaboratus (Brunner von Wattenwyl, 1907) - Otte \& Brock 2005: 301.

MATÉRIEL TYPE AU MNHN. - HT $q$.

HT ㅇ (MNHN-EO-PHAS410): [Vietnam], Tonkin centr[e], Env[irons] de Tuyen-Quan, A. Weiss, 1901.

\section{ensis Bolívar, 1922 [Gratidia]}

Gratidia ensis Bolívar, 1922: 209.

Statut actuel. - Clonaria ensis (Bolívar, 1922). - Otte \& Brock 2005: 100

MatéRIEL TYPE AU MNHN. -2 o ST.

1 ơ ST (MNHN-EO-PHAS74)] : Ethiopie Mérid[ionale], Filoa-

Tchoba, Maurice de Rothchild, avril, 1905.

1 ơ ST (MNHN-EO-PHAS75) : Ethiopie Mérid[ionale], Bourka, Maurice de Rothchild, avril, 1905.

\section{erosus Redtenbacher, 1906 [Chitoniscus]}

Chitoniscus erosus Redtenbacher, 1906: 179.

STATUT ACTUEL. - Chitoniscus erosus Redtenbacher, 1906.

MatéRIEL TYPE AU MNHN. - 1 \% ST.

1 ㅇ ST (MNHN-EO-PHAS1018): N[ouv]elle-Calédonie, Canal Woodia, Dr François, 783-[18]92.
REMARQUE

1 \& ST au NHMW; la 9 ST du HNHM a été détruite dans un incendie (Brock 1998a, 2007).

\section{erringtoniae Redtenbacher, 1906 [Heteropteryx]}

Heteropteryx erringtoniae Redtenbacher, 1906: 169.

STATUT ACTUEL. - Haaniella erringtoniae (Redtenbacher, 1906) Hennemann et al. 2016a: 35.

Matériel TYPE AU MNHN. - HT 9.

HT (MNHN-EO-PHAS1019): [Malaisie], Pr[ovince] Malacca, Selangor, Errington et Chapé, 1900.

\section{erythropterus Redtenbacher, 1906 [Damasippoides]}

Damasippoides erythropterus Redtenbacher, 1906: 158.

STATUT ACTUEL. - Damasippoides erythropterus Redtenbacher, 1906.

MATÉRIEL TYPE AU MNHN. $-20^{7}$ ST.

20 OT (MNHN-EO-PHAS910, MNHN-EO-PHAS911) : Madagascar, Maroancetra [Maroantsetra], 12.1897, collection Finot.

\section{excelsa Redtenbacher, 1908 [Necroscia]}

Necroscia excelsa Redtenbacher, 1908: 559.

StatuT aCtUel. - Neonescicroa excelsa (Redtenbacher, 1908) Seow-Choen 2016: 177.

MATÉRIEL TYPE AU MNHN. -2 9 ST.

1 \& ST (MNHN-EO-PHAS372) : [Indonésie], Bornéo, M. Chaper, $1891,1280[18] 91,34$.

1 \& ST (MNHN-EO-PHAS373) : [Indonésie], Bornéo, M. Chaper, 1891,1280 [18]91, 117.

\section{REMARQUE}

Chaper a exploré la partie occidentale de Bornéo, autour du fleuve Kapocas (Kapuas), donc actuellement dans la partie indonésienne de l'île (Kalimatan). 1 \& ST au NHMW (Brock, 1998a).

\section{exiguus Redtenbacher, 1908 [Tropidoderus]}

Tropidoderus exiguus Redtenbacher, 1908: 384.

STATUT ACTUEL. - Tropidoderus exiguus Redtenbacher, 1908.

MatéRIEL TYPE AU MNHN. — LT ơ, $10^{7}$ PLT.

LT ơ (MNHN-EO-PHAS451) (présente désignation): Patria ignota, $570[18] 73,34$.

$10^{7}$ PLT (MNHN-EO-PHAS452): Patria ignota, 570 [18]73.

REMARQUE

Nous avons désigné un LT parmi les deux ST existants en choisissant le spécimen correspondant le plus à la description par sa morphologie et sa taille. 
exsiccata Redtenbacher, 1906 [Onogastris]

Onogastris exsiccata Redtenbacher, 1906: 24.

STATUT ACTUEL. - Onogastris pardalina Redtenbacher, 1906: 24, pl. 2, figs 10, 11 - Cliquennois 2003a (synonymie).

MATÉRIEL TYPE AU MNHN. - HT 9.

HT $९$ (juvénile) (MNHN-EO-PHAS111): Madagascar, région du sud-est, Vallée d'Ambolo, Col de Sakavalana, Ch. Alluaud, Janv[ier] 01, [19]01.

femorata Hennemann, Conle, Bellanger, Lelong \& Jourdan, 2018 [Phantasca]

Phantasca femorata Hennemann, Conle, Bellanger, Lelong \& Jourdan, 2018: 14, figs 16-24.

Statut actuel. - Phantasca femorata Hennemann, Conle, Bellanger, Lelong \& Jourdan, 2018.

Matériel TYPe AU MNHN. - HT $\$, 1$ PT.

HT $\%$ (MNHN-EO-PHAS1222): [Guyane], P. E. Roubaud det., 25.VII, A7 N12, [coll. GEP].

1 ㅇ PT (MNHN-EO-PHAS1223) : [Guyane], P. E. Roubaud det. 25.VII, A6 S1 N7, 1 [coll. GEP].

\section{REMARQUE}

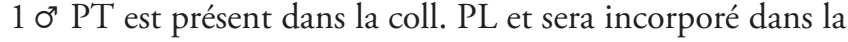
collection du MNHN; 1 ơ PT dans la collection OC (Hennemann et al. 2018).

\section{festinabunda Redtenbacher, 1908 [Ocellata]}

Ocellata festinabunda Redtenbacher, 1908: 555.

STATUT ACTUEL. - Orthonecroscia festinabunda (Redtenbacher, 1908) - Bragg 2001: 585.

Matériel TYPe aU MNHN. - HT ơ.

HT ơ (MNHN-EO-PHAS1020) : [Malaisie], Nord Bornéo, J. Whitehead, 917-[18]83.

\section{fictus Redtenbacher, 1908 [Paramyronides]}

Paramyronidesfictus Redtenbacher, 1908: 478.

StATUT ACTUEL. - Parasipyloidea ficta (Redtenbacher, 1908). Giglio-Tos 1910: 46.

MATÉRIEL TYPE AU MNHN. - HT o".

HT ơ (MNHN-EO-PHAS1021): [Inde], Sikkim, J. Harmand, 3041-[18]86

\section{REMARQUE}

Le nom générique étant féminin, la terminaison -a s'impose pour l'épithète spécifique (accord rectifié).

\section{finitima Brunner von Wattenwyl, 1907 [Pachymorpha]}

Pachymorpha finitima Brunner von Wattenwyl, 1907: 215.
STATUT ACTUEL. - Tectarchus huttoni (Brunner von Wattenwyl, 1907): 213 - Brock 1997: 21 (synonymie).

MATÉRIEL TYPE AU MNHN. - 1 ST $q$.

1 ST (MNHN-EO-PHAS439): Nouv[elle]-Zélande, Petit, 48[18]52, 48 [18]52, 1.

REMARQUE

1 오 ST au NHMW (Brock 1998a; Brock et al. 2018).

\section{finoti Redtenbacher, 1906 [Cirsia]}

Cirsia finoti Redtenbacher, 1906: 22, pl. 2, fig. 7.

STATUT ACTUEl. - Cirsia finoti Redtenbacher, 1906.

Matériel type AU MNHN. — LT ơ, 1 9 PLT.

LT ơ (MNHN-EO-PHAS947), 1 ○ PLT (MNHN-EO-PHAS948): Madagascar, collection Finot.

REMARQUE

1 PLT au NHMW (Cliquennois 2003a). Le LT a été désigné par Cliquennois (2003a: 8).

\section{flavescens Sjöstedt, 1909 [Gratidia]}

Gratidia flavescens Sjöstedt, 1909: 87.

Statut aCtUel. - Clonaria flavescens (Sjöstedt, 1909) - Otte \& Brock 2005: 100

MATÉRIEL TYPE AU MNHN. - 1 ㅇ ST.

1 o ST (MNHN-EO-PHAS40): [Tanzanie], Kilimandjaro, Kilimandjaro et Mérou, Expédit[tion] Suédoise, Sjöstedt, Y. Sjöstedt, sept[embre], 1905-[190]6, 1912.

REMARQUE

3 ㅇ ST au NHRS, 1 ㅇ ST au ZMB (Brock et al. 2018).

flaviceps Redtenbacher, 1906 [Orobia]

Orobia flaviceps Redtenbacher, 1906: 73.

STATUT ACTUEL. — Leiophasma flaviceps (Redtenbacher, 1906) Brock 1998a: 29.

MATÉRIEL TYPE AU MNHN. - $50^{7}$ ST, 2 9 ST.

$10^{7}$ ST (MNHN-EO-PHAS113) : Madagascar, 1869, 198 [18]69, 199.

1 O’ ST (MNHN-EO-PHAS253) : Madagascar, Maroancetra [Maroantsetra], XII.1897, collection Finot.

3 o' ST (MNHN-EO-PHAS1022, MNHN-EO-PHAS1023, MNHN-EO-PHAS1024) et 2 \& ST (MNHN-EO-PHAS1025, MNHN-EO-PHAS1026) : Madagascar, 1262-[18]78.

REMARQUE

Le ST MNHN-EO-PHAS253 appartient en fait à Leiophasma vaginatum (Redtenbacher, 1906). 2 o' ST et 1 \% ST au NHMW, 1 ơ ST à l'ISNB (Brock 1998a). 


\section{flavipes Chopard, 1911 [Phasma]}

Phasma flavipes Chopard, 1911: 339, fig. B.

STATUT ACTUEL. - Pseudophasma flavipes (Chopard, 1911) - Otte \& Brock 2005: 289.

MATÉRIEL TYPe AU MNHN. - 3 O' ST, 1 \% ST.

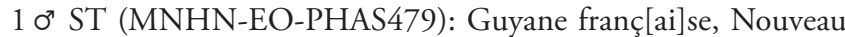
chantier, collection Le Moult, collection Lucien Chopard, 191.

1 o ST (MNHN-EO-PHAS482): Guyane franç[ai]se, S[ain] t-Laurent du Maroni, novembre, collection Le Moult, collection Lucien Chopard, 191.

207 ST (MNHN-EO-PHAS480, MNHN-EO-PHAS481) : [sans localité], collection Lucien Chopard, 191.

\section{REMARQUE}

Chopard (1911) mentionne $30^{7}$ et 1 \% syntypes, ce qui correspond au total des spécimens présentement répertoriés. Le ơ ST mentionné par Brock et al. (2018) à l'ANSP ne peut donc pas être un type.

\section{flavomarginata Redtenbacher, 1908 [Marmessoidea]}

Marmessoidea flavomarginata Redtenbacher, 1908: 512.

STATUT ACTUEL. - Marmessoidea flavomarginata Redtenbacher, 1908.

MatéRIEL TYPe AU MNHN. -20 ST.

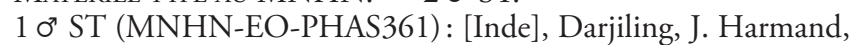
2854.[18]90, 59.

1 o' ST (MNHN-EO-PHAS362) : [Inde], Darjiling, J. Harmand, 2854.[18]90.

\section{foliacea Blanchard, 1851 [Bacteria]}

Bacteria foliacea Blanchard, 1851a: 26.

STATUT ACTUEL. - Heteronemia mexicana Gray, 1835: 19 - Zompro 2001: 223 (synonymie).

MATÉRIEL TYPE AU MNHN. - HT ơ.

HT ơ (MNHN-EO-PHAS1071): Chili, C. Gay, 15-[18]43, 15 [18]42, 21.

\section{REMARQUES}

Une étiquette de détermination (que nous supposons la plus ancienne) indique: "Bacteria foliacea Blanch.". Une autre étiquette est de Brunner von Wattenwyl: «21. Donusa prolixa Stål». Le spécimen est collé sur une plaque cartonnée avec quelques morceaux de pattes. Camousseight a noté sur le côté droit de ce morceau de carton: "Bacunculus granulicollis (Bl.), dét. Camousseight, 1986» et estimait donc qu'il s'agissait d'un Heteronemia granulicollis (Blanchard, 1851) (Heteronemia Gray, 1835 = Bacunculus Burmeister, 1838). Le spécimen dont le corps est entier, n'a pratiquement plus d'appendices. Ainsi, il ne lui reste guère qu'un petit morceau de l'antenne droite, une patte médiane droite mais sans les tarses et un autre petit morceau de patte sur la feuille cartonnée sur laquelle est épinglé le spécimen. En outre, il s'agit d'un ơ juvénile. Pourtant, les caractères observables permettent de confirmer qu'il s'agit bien d'un Heteronemia. Les anciennes étiquettes attestent qu'il s'agit du type de Blanchard, de même que ses mensurations qui correspondent parfaitement à celles énoncées par Blanchard (1851a).

\section{fortior Brunner von Wattenwyl, 1907 [Ocnophila]}

Ocnophila fortior Brunner von Wattenwyl, 1907: 315.

STATUT ACTUEL. — Ocnophila fortior Brunner von Wattenwyl, 1907.

MATÉRIEL TYPE AU MNHN. - 1 ST ơ.

1 ST ơ (MNHN-EO-PHAS627) : [Brésil], Montagne des Orgues, Prov[ince] de Rio [de] Janeiro, Massif de Tijuca, 500 à 900 m d'alt., E. R. Wagner, d'Octobre 1901 à Mars 1902.

\section{REMARQUE}

3 juvéniles avec les mêmes étiquettes que le ST sont également présents dans la collection (MNHN-EO-PHAS628, MNHN-EO-PHAS629, MNHN-EO-PHAS630). 1 ST o" au NHMW (Brock 1998a; Brock et al. 2018).

\section{fritzschei Zompro, 2000 [Gratidia]}

Gratidia fritzschei Zompro, 2000a: 53, figs 9-21.

STATUT ACTUEl. - Clonaria fritzschei (Zompro, 2000) - Otte \& Brock 2005: 100.

Matériel type AU MNHN. - HT ơ, 1 \% PT.

HT ơ (MNHN-EO-PHAS392) : Thaïlande, Nakhon Ratchasima, Amphoe Pak Chong, Thanon Thannarat, km 6, 101 $21^{\circ} \mathrm{E} 14^{\circ} 32^{\prime} \mathrm{N}$, 325 m, 21.XI.1997, 358-54, Coll. OZ.

1 ㅇ PT (MNHN-EO-PHAS393): Thaillande, Nakhon Ratchasima, Amphoe Pak Chong, Thanon Thannarat, km 7, 101 $24^{\prime} 50 \mathrm{E}$ $14^{\circ} 36^{\prime} 50 \mathrm{~N}, 300$ m, 17.XII.1997, 358-54, Coll. OZ.

\section{REMARQUE}

$10^{7}$ et 1 ? PT au NHMUK; CUMZ; DEIC; MSNG; $10^{7}$ et 1 P PT au NHMW; $10^{7}$ et 1 \& PT à l'OXUM; SMFD; MTD; UMB; $10^{*}$ et 1 P PT au ZMB; $10^{\prime \prime}$ et 1 ㅇ PT au ZMH; $10^{7}$ et 1 ㅇ PT au MHNG; $10^{7}$ PT dans la collection OZ (Zompro 2000a, 2002b \& 2003; Zompro \& Brock 2003; Brock et al. 2018).

\section{frubstorferi Brunner von Wattenwyl, 1907 [Clitumnus]}

Clitumnus fruhstorferi Brunner von Wattenwyl, 1907: 193.

STATUT ACTUEL. - Ramulus fruhstorferi (Brunner, 1907) - Otte \& Brock 2005: 302.

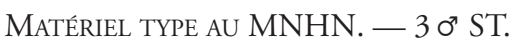

2 ơ ST (MNHN-EO-PHAS411, MNHN-EO-PHAS412) : [Vietnam], Tonkin Centr., Env[irons] de Tuyen-Quan, Printemps, A. Weiss, 1901.

1 ơ ST (MNHN-EO-PHAS1027): [Vietnam], Tonkin, région haute rivière claire, $\mathrm{A}$. Weiss. 
REMARQUE

$70^{7}$ ST au NHMW (Brock et al. 2018) ainsi que 4 o" sans étiquettes comme étant autant de types potentiels (Brock 1998a).

\section{furcatus Brunner von Wattenwyl, 1907 [Dixippus]}

Dixippus furcatus Brunner von Wattenwyl, 1907: 279.

STATUT ACTUEL. - Periphetes forcipatus (Bates, 1865) : 338 - Hennemann \& Conle 2007: 53 (synonymie).

Matériel TYPe AU MNHN. - HT ơ.

HT ơ (MNHN-EO-PHAS330): [Indonésie], Célèbes, de la Savinière, 2085-[18]77, 70.

\section{furcifer Sjöstedt, 1909 [Gratidia]}

Gratidia furcifer Sjöstedt, 1909: 87, fig. 7.

STATUT ACTUEl. - Clonaria furcifer (Sjöstedt, 1909) - Otte \& Brock 2005: 101

MatéRIEL TYPE AU MNHN. - 10 O ST.

1 ơ ST (MNHN-EO-PHAS49) : [Tanzanie], Meru, Nieder, Ngare na nyuki, Kilimandajaro et Mérou, Expédit[ion] Suédoise, Sjöstedt, Y. Sjöstedt, jan[vier], 1912.

\section{REMARQUE}

4 ơ ST au NHRS (K. A. Johanson comm. pers.).

\section{furcillatus Pantel, 1917 [Carausius]}

Carausius furcillatus Pantel, 1917: 267, pl. 2, figs 1, 2.

Statut aCtUel. - Carausius furcillatus Pantel, 1917.

MATÉRIEL TYPe AU MNHN. - $10^{7}$ ST, 4 9 ST.

1 ơ ST (MNHN-EO-PHAS680) : possible ponte parthén[o] g[énétique], 1908, [collection Pantel].

1 \& ST (MNHN-EO-PHAS681) : [Inde], Shembaganur, J. Dubreuil, Shembaganur, 1, [collection Pantel].

1 ㅇ ST (MNHN-EO-PHAS682) : 2, [collection Pantel].

1 \& ST (MNHN-EO-PHAS683) : [Inde], Mission Maduré, struma nulla, 3, [collection Pantel].

1 o ST (MNHN-EO-PHAS684): [Inde], Shembaganur, J. Dubreuil, struma nulla, vertes appendice, 4, 33, [collection Pantel].

\section{REMARQUE}

Pantel (1917) citait peu de ơ (sans précision du nombre), mais mentionnait les 4 ㅇ répertoriées ici. Deux autres 9 (MNHNEO-PHAS685, MNHN-EO-PHAS686), sont visiblement issues d'élevage (étiquette "nigrine expérimental») et ne sont pas à prendre en compte.

\section{fuscipennis Redtenbacher, 1908 [Bactrododema]}

Bactrododema fuscipennis Redtenbacher, 1908: 392, pl. 18, fig. 4.

STATUT ACTUEL. - Dematobactron fuscipennis (Redtenbacher, 1908) - Karny 1923: 239
Matériel TYPe AU MNHN. -2 \% ST.

2 ㅇ ST (MNHN-EO-PHAS1094, MNHN-EO-PHAS1095) : Gabon, P. A. Le Roy, 1893.

REMARQUE

2 ㅇ ST au NHMW ; 2 ㅇ ST au SMNS (Brock 1998a; Hennemann \& Conle 2003; Zompro 2006).

\section{gaudichaudi Redtenbacher, 1908 [Heterophasma]}

Heterophasma gaudichaudi Redtenbacher, 1908: 386, pl. 19, fig. 2. STATUT ACtUel. - Heterophasma gaudichaudi Redtenbacher, 1908. Matériel TYPE AU MNHN. - HT 9.

HT ㅇ (MNHN-EO-PHAS1092) : Madagascar, Expédition de la Bonite, C. Gaudichaud, 951-[18]37.

\section{gibbosa Chopard, 1911 [Phibalosoma]}

Phibalosoma gibbosa Chopard, 1911: 347, fig. [sans numéro].

STATUT ACTUEL. - Cladomorphus gibbosus (Chopard, 1911) Otte \& Brock 2005: 94.

MATÉRIEL TYPE AU MNHN. - HT $\%$

HT 9 juvénile (MNHN-EO-PHAS548): Guyane franç[aise], S[ain] t-Laurent du Maroni, novembre, collection Le Moult.

gigantea saintluciae Hennemann, Conle, \& PerezGelabert, 2016 [Diapherodes]

Diapherodes gigantea saintluciae Hennemann, Conle \& Perez-Gelabert, 2016b: 80, figs 136-143, 367, 381.

STATUT ACTUEL. - Diapherodes gigantea saintluciae Hennemann, Conle \& Perez-Gelabert, 2016.

Matériel TyPe au MNHN. - 1 o PT, 1 \% PT.

$10^{7}$ PT (MNHN-EO-PHAS1115): Sainte-Lucie, Barre de l'Isle Trail, Alt. 275-305 m, E. Dorel, F. Langlois \& P. Lelong, 2.XI.2003, SLU03-090, collection ASPER.

1 ㅇ PT (MNHN-EO-PHAS1116): Sainte-Lucie, Barre de l'Isle Trail, Alt. 275-305 m, E. Dorel, F. Langlois \& P. Lelong, 2.XI.2003, SLU03-094, collection ASPER.

\section{REMARQUE}

HT $9,30^{7}$ PT et 2 PT au NHMUK; $10^{7}$ PT au FSCA (Hennemann et al. 2016b). Seul un couple est actuellement au MNHN, les 3 ơ PT et 4 o PT sont actuellement dans la coll. ASPER et viendront rejoindre la collection nationale du MNHN dans l'avenir.

\section{gracilipes Redtenbacher, 1908 [Asprenas]}

Asprenas gracilipes Redtenbacher, 1908: 349.

StatuT aCtUel. - Asprenas gracilipes Redtenbacher, 1908.

MATÉRIEL TYPE AU MNHN. - HT o

HT ơ (MNHN-EO-PHAS459) : N[ouv]elle-Calédonie, Bourail, H. Méray, 1902. 
gracilis Hennemann \& Conle, 2004 [Achrioptera]

Achrioptera gracilis Hennemann \& Conle, 2004: 16, figs 15, 2628, 64-65, 87.

STATUT ACTUEL. - Achrioptera gracilis Hennemann \& Conle, 2004.

MATÉRIEL TYPE AU MNHN. - HT $q$.

HT (MNHN-EO-PHAS86): Madagascar, Ivohibe, $1500 \mathrm{~m}, \mathrm{R}$. Decary, 1926.

\section{gracilis Chopard, 1938 [Gratidia]}

Gratidia gracilis Chopard, 1938: 116.

STATUT ACtuel. - Clonaria gracilis (Chopard, 1938) - Otte \& Brock 2005: 101.

MATÉRIEL TYPE AU MNHN. — HT ơ, AT $\%$.

HT ơ (MNHN-EO-PHAS22): Kenya, Mission de l'Omo, Camp Cherangani, Marakwet, 3000 m, C. Arambourg, P. A. Chappuis \& R. Jeannel, 1932-[19]33.

AT ? (MNHN-EO-PHAS23): Kenya, Mission de l'Omo, Camp Cherangani, Marakwet, 3500 m, C. Arambourg, P. A. Chappuis \& R. Jeannel, 1932-[19]33.

\section{REMARQUE}

6 autres $0^{\prime \prime}$ (MNHN-EO-PHAS24, MNHN-EO-PHAS25, MNHN-EO-PHAS246, MNHN-EO-PHAS27, MNHNEO-PHAS28, MNHN-EO-PHAS29) sont de la même série mais ne sont pas des types car Chopard (1938) n'en fait pas mention: Kenya, Mission de l'Omo, Kitale, Uasin Gishu, 1932-33, 2100 m, C. Arambourg, P. A. Chappuis \& R. Jeannel.

\section{graminis Sjöstedt, 1909 [Gratidia]}

Gratidia graminis Sjöstedt, 1909: 81.

StatuT ACtUel. - Clonaria graminis (Sjöstedt, 1909) - Otte \& Brock 2005: 101.

MATÉRIEL TYPE AU MNHN. - 1 \% ST.

1 으 ST (MNHN-EO-PHAS39): [Tanzanie], Kilimandjaro, Kilimandjaro et Mérou, Expédit[ion] Suédoise, Sjöstedt, Y. Sjöstedt, sept[embre], 1905-[190]6, 1912.

\section{REMARQUE}

Sjöstedt (1909) évoque de nombreux syntypes sans préciser leur nombre. Sjöstedt n'a décrit que les $\$$ de cette espèce et de ce fait le spécimen ơ (MNHN-EO-PHAS38) présent dans la collection ne peut être un type. 10 spécimens sont répertoriés du NHRS (K. A. Johanson comm. pers.) dont au moins 3 ST ㅇ. 1 ST ㅇ au ZMHB (Brock et al. 2018).

\section{granulatus Brullé, 1832 [Bacillus]}

Bacillus granulatus Brullé, 1832: 84, pl. 29, fig. 6.

STATUT ACTUEL. - Clonopsis gallica (Charpentier, 1845) - Burmeister 1840: 40 (synonymie); Otte \& Brock, 2005: 108 (nouvelle combinaison).
Matériel TYPe au MNHN. - HT 9 .

HT 9 (MNHN-EO-PHAS1135) : [Grèce], Morée, A. Brullé, 4187-

[18]33, 48, 47, [ex-collection A. Lefevre].

\section{griveaudi Paulian, 1960 [Achrioptera]}

Achrioptera griveaudi Paulian, 1960: 272, fig. 1.

STATUT ACTUEL. - Achrioptera griveaudi Paulian, 1960.

Matériel tYPE AU MNHN. - HT 9,1 PT.

HT $ᄋ$ (MNHN-EO-PHAS129), 1 ㅇ PT (MNHN-EO-PHAS130):

[Comores], Mohéli, Bandamalé, 420 m, E. Raharizonina, XI-

[19]58, dans l'estomac de Leptosomus discolor, Institut scientifique [de]Madagascar.

\section{guianensis Hennemann \& Conle, 2007 [Jeremiodes]}

Jeremiodes guianensis Hennemann \& Conle, 2007: 6, figs 4-10, 12, 13.

STATUT ACTUEL. - Jeremiodes guianensis Hennemann \& Conle, 2007.

MatéRIEL TYPE AU MNHN. - HT ơ, 2 \% PT.

HT ơ (MNHN-EO-PHAS545), 1 \& PT (MNHN-EO-PHAS546): Guyane française, Montagnes de Kaw, Piste de Kaw, P. E. Roubaud, [Coll. GEP].

1 P PT juvénile (MNHN-EO-PHAS547): Guyane française, Montagnes de Kaw, Piste de Kaw, J. Solard, P. E. Roubaud, [Coll. GEP].

guianensis Hennemann, Conle, Bellanger, Lelong \& Jourdan, 2018 [Phantasca]

Phantasca guianensis Hennemann, Conle, Bellanger, Lelong \& Jourdan, 2018: 18, figs 25-41.

Statut actuel. - Phantasca guianensis Hennemann, Conle, Bellanger, Lelong \& Jourdan, 2018.

Matériel tYPe AU MNHN. - HT ơ, 1 PT.

HT ơ (MNHN-EO-PHAS1220: Guyane, Montagne de Kaw, Roubeaud, Auvray, Rarchaert rec., 5-12.VIII.[19]92, 8K 37 A4, [coll. GEP].

1 \& PT (MNHN-EO-PHAS1221): Guyane, Piste de Kaw, Roubaud, Rarchaert, Morin, Auvray rec., 5.VII.[19]92, 5 [coll. GEP].

\section{REMARQUE}

Deux autres PT + sont présents dans la coll. PL et les œufs associés dans la collection coll. YB et incorporeront les collections du MNHN dans l'avenir.

\section{harpyia Redtenbacher, 1908 [Canachus]}

Canachus harpyia Redtenbacher, 1908: 346.

Statut aCtUel. - Canachus harpyia Redtenbacher, 1908.

Matériel TYPE AU MNHN. - HT 9 .

HT \& (MNHN-EO-PHAS1072): Nouv[elle]-Calédonie, 1875,

R. Germain, "Aire du sud, Bois mort», n45, 133 [18]75, 175. 
hilaris Brunner von Wattenwyl, 1907

[Carausius]

Carausius hilaris Brunner von Wattenwyl, 1907: 268.

STATUT ACTUEL. - Carausius hilaris Brunner von Wattenwyl, 1907.

MATÉRIEL TYPE AU MNHN. - 3 \% ST.

1 9 ST (MNHN-EO-PHAS687) : [Inde], Mission Maduré, P. Décoly, 1897, n 196, [collection Pantel].

1 ㅇ ST (MNHN-EO-PHAS688): [Inde], Shembaganor, Mission Maduré, J. Dubreuil, 1901, S. J. Mallat, [collection Pantel].

1 ㅇ ST (MNHN-EO-PHAS689) : [Inde], Shembaganor, J. Dubreuil, S. J. Mallat, 1901, 33, [collection Pantel].

\section{horrida Boisduval, 1835 [Eurycantha]}

Eurycantha horrida Boisduval, 1835: 647, pl. 10, fig. 2.

StatuT ACtUel. - Eurycantha horrida Boisduval, 1835.

Matériel TYPe AU MNHN. -207 ST.

$20^{7}$ ST (MNHN-EO-PHAS472, MNHN-EO-PHAS473) : [Nouvelle-Guinée] N[ouve]lle Hollande, Dumont d'Urville.

\section{REMARQUE}

Boisduval (1835) mentionne un ou plusieurs mâles de «l'océan Pacifique» rapportés par Labillardière et un ou plusieurs autres spécimens mâles de Doreï (Manokwari) en Nouvelle-Guinée rapportés par Dumont d'Urville. Nous n'avons pas retrouvé le moindre spécimen de Labillardière. En revanche, deux spécimens de Dumont d'Urville correspondant à la description sont bien présents au MNHN. Leurs étiquettes mentionnent les données suivantes : "Nlle Hollande, Dumont d'Urville». Dans la boîte, étalée sous les deux spécimens se trouve une étiquette typique de Redtenbacher, "Eurycantha horrida Boisd", lequel a donc vu les spécimens (cf. Redtenbacher 1908: 342), étiquette malencontreusement ôtée de l'épingle d'un des deux spécimens; et une étiquettre rose très ancienne: «E. horrida Boisd, Nlle Hollande, M. d'Urville». La mention de la Nouvelle-Hollande (l'Australie, où E. horrida n'est pas connue - Brock \& Hasenpusch 2009) apparaît erronée et est probablement le résultat d'une confusion lors de l'étiquetage avec l'île de Nouvelle-Irlande, où se situe justement Doreï, ce qui nous conduit à considérer ces deux spécimens comme des syntypes.

\section{huttoni Brunner von Wattenwyl, 1907 [Pachymorpha]}

Pachymorpha huttoni Brunner von Wattenwyl, 1907: 213.

STATUT ACTUEL. - Tectarchus huttoni (Brunner von Wattenwyl, 1907) - Brunner von Wattenwyl 1907: 213 - Brock 1997: 21.

MATÉRIEL TYPE AU MNHN. - 10 o ST, 1 \% ST.

1 ơ ST (MNHN-EO-PHAS437): Nouv[elle]-Zélande, Nelson, H. Filhol, 1876, 797 [18]76, 49.

1 ㅇ ST (MNHN-EO-PHAS438): Nouv[elle]-Zélande, Nelson, H. Filhol, 1876, 717 [18]76, 29, 29.
REMARQUE

2 ot $10^{7}$ ST au NHMW (Brock 1998a).

illaesa Redtenbacher, 1908 [Necroscia]

Necroscia illaesa Redtenbacher, 1908: 564.

STATUT ACTUEL. - Trachythorax maculicollis (Westwood, 1848) Westwood 1848: 77, pl. 38, fig. 2 - Brock \& Delfosse 2005: 52 (synonymie).

Matériel type au MNHN. - HT o7.

HT ơ (MNHN-EO-PHAS800) : [Inde], Shembagan[ur], P. Décoly, VIII.[19]00, No 3 [collection Pantel].

imbellis Brunner von Wattenwyl, 1907 [Carausius]

Carausius imbellis Brunner von Wattenwyl, 1907: 269.

Statut aCtUel. — Carausius imbellis Brunner von Wattenwyl, 1907.

Matériel TYPe AU MNHN. - 3 9 ST, 1 o ST.

1 ㅇ ST (MNHN-EO-PHAS734): [Inde], Mission Maduré, [collection Pantel].

1 ㅇ ST (MNHN-EO-PHAS735): [Inde], Kod[aikanal], Décoly, [collection Pantel].

1 ㅇ ST (MNHN-EO-PHAS737) (juvénile): [Inde], Kodikanel [Kodaikanal], P. Décoly, [collection Pantel].

1 ơ ST (MNHN-EO-PHAS738) : [Inde], Ind[es] Or[ientales], P.

Castets, [collection Pantel].

\section{REMARQUE}

D'autres ST au MNMS (Brock \& Delfosse 2005).

impennis Redtenbacher, 1908 [Achrioptera]

Achrioptera impennis Redtenbacher, 1908: 441.

STATUT ACTUEL. - Achrioptera impennis Redtenbacher, 1908.

MATÉRIEL TYPE AU MNHN. - HT ơ.

HT ơ (subadulte) (MNHN-EO-PHAS87) : Madagascar, région de l'Androy, Ambovombe, Dr J. Decorse, mars [19]01, 1901.

imperialis Brunner von Wattenwyl, 1907

[Cuniculina]

Cuniculina imperialis Brunner von Wattenwyl, 1907: 204.

STATUT ACTUEL. - Ramulus imperialis (Brunner von Wattenwyl, 1907) - Otte \& Brock 2005: 303.

MATÉRIEL TYPE AU MNHN. - 1 9 ST.

1 ㅇ ST (MNHN-EO-PHAS413): [Laos], [montagnes de] Lakhon, J. Harmand, 1878, 991 [18]78, 77.

REMARQUE

1 O ST au NHMW (Brock 1998a). Cette espèce serait à placer parmi les Medauroidea (J. Bresseels comm. pers.). 
impigra Brunner von Wattenwyl, 1907 [Cuniculina]

Cuniculina impigra Brunner von Wattenwyl, 1907: 204.

STATUT ACTUEL. - Ramulus impiger (Brunner von Wattenwyl, 1907) - Otte \& Brock 2005: 303 [Ramulus impigrus (Brunner von Wattenwyl, 1907) (sic)].

Matériel TYPe AU MNHN. - 1 9 ST.

1 ㅇ ST (MNHN-EO-PHAS414): [Vietnam], Tonkin Centr[e], Env[irons] de Tuyen-Quan, A. Weiss, Printemps, 1901.

REMARQUE

3 \& ST au NHMW; 8 autres ST possibles à l'ISNB, sans précision (Brock 1998a). La forme masculine «impigrus» est incorrecte (accord rectifié).

\section{incisa Chopard, 1938 [Gratidia]}

Gratidia incisa Chopard, 1938: 116.

StATUT ACTUEL. - Clonaria incisa (Chopard, 1938) - Otte \& Brock 2005: 101.

MATÉRIEL TYPE AU MNHN. - HT ơ.

HT ơ" (MNHN-EO-PHAS16) : Kenya, Mission de l'Omo, Camp III de l'Elgon, Zone des bruyères, Est, 3500 m, C. Arambourg, P.-A. Chappuis \& R. Jeannel, 1932-[19]33.

\section{REMARQUE}

Un autre spécimen ơ (MNHN-EO-PHAS17) de la même série n'est pas cité par Chopard (1938) et n'est donc pas un type.

inconspicuus Brunner von Wattenwyl, 1907 [Bacunculus]

Bacunculus inconspicuus Brunner von Wattenwyl, 1907: 334.

STATUT ACtUel. - Pseudosermyle inconspicua (Brunner von Wattenwyl, 1907) - Zompro 2001: 216.

MATÉRIEL TYPE AU MNHN. - 1 ST o".

1 ST ơ (MNHN-EO-PHAS597): Mexique, Mozard.

\section{REMARQUE}

1 ST $0^{7}$ au NHMW; 2 ST ơ au MHNG (Brock 1998a; Zompro \& Brock 2003).

\section{infans Redtenbacher, 1906 [Pylaemenes]}

Pylaemenes infans Redtenbacher, 1906: 49.

STATUT ACTUEL. - Pylaemenes otys (Westwood, 1859) - Westwood 1859: 54, pl. 26, fig. 2 - Bragg 1998: 75 (synonymie); Zompro 2004: 226 (statut actuel).

Matériel TYPe Au MNHN. - HT ơ.

HT ơ (MNHN-EO-PHAS342) : Bornéo, R. Oberthür, 1898, 10,10 . inquinatus Redtenbacher, 1908 [Asceles]

Asceles inquinatus Redtenbacher, 1908: 498.

Statut actuel. - Asceles inquinatus Redtenbacher, 1908.

Matériel tyPe au MNHN. - 10 $0^{\text {T }}$ PLT.

$10^{7}$ PLT (MNHN-EO-PHAS378): [Indonésie], Bornéo, M. Chaper, 1891,1286 [18]91, 89.

\section{REMARQUE}

Chaper a exploré la partie occidentale de Bornéo, autour du fleuve Kapocas (Kapuas), donc actuellement dans la partie indonésienne de l'île (Kalimatan). LT,+ 2 o et $40^{7}$ PLT au NHMW ; 8 \% PLT au ZIN (Brock 1998a; Brock et al. 2018). Le LT a été désigné par Brock (1995: 87).

insolens Brunner von Wattenwyl, 1907 [Carausius]

Carausius insolens Brunner von Wattenwyl, 1907: 268.

STATUT ACTUEL. - Carausius insolens Brunner von Wattenwyl, 1907.

MATÉRIEL TYPE AU MNHN. - $20^{\pi}$ ST, 2 ㅇ ST.

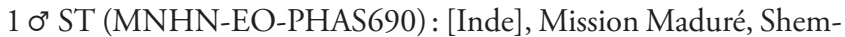
baganor [Shembaganur], $0^{7}$ anth., [collection Pantel].

1 ơ ST (MNHN-EO-PHAS691), 1 O ST (MNHN-EO-PHAS693):

[Inde], Shembaganur, J. Dubreuil, 30, bande blanche, [collection Pantel].

1 ㅇ ST (MNHN-EO-PHAS692): [Inde], Mission Maduré, 30, $0^{7} O$, anth., [collection Pantel]

insolens Brunner von Wattenwyl, 1907 [Cuniculina]

Cuniculina insolens Brunner von Wattenwyl, 1907: 201.

STATUT ACTUEl. - Cuniculina cuniculus (Westwood, 1859) Hennemann 2002: 65 (synonymie) [Cuniculina cunicula (Westwood, 1859) sic].

MATÉRIEL TYPE AU MNHN. - 1 ơ ST, 1 \& ST.

$10^{7}$ ST (MNHN-EO-PHAS405): [Inde], Indes anglaises, Balasore,

R. Oberthür, 1898, 16, $16=1$.

REMARQUE

Dans la description originale, Brunner von Wattenwyl (1907) décrit la ㅇ de Colombo (Sri Lanka), puis «ajoute» le $\sigma^{7}$ de "l'Inde anglaise", tout en donnant les mensurations d'une ․ 1 ST au NHMW (Brock 1998a). L'épithète spécifique "cuniculus» est un substantif et à ce titre invariable (accord rectifié).

insolita Brunner von Wattenwyl, 1907 [Gratidia]

Gratidia insolita Brunner von Wattenwyl, 1907: 227.

STATUT ACTUEL. - Clonaria insolita (Brunner von Wattenwyl, 1907) - Otte \& Brock 2005: 101.

Matériel tYPE au MNHN. - HT 9 .

HT ? (MNHN-EO-PHAS1028) : Congo, M. Tholon, 1001-1892. 


\section{REMARQUES}

Il existe deux autres spécimens + : le premier (MNHN-EOPHAS1206) avec une étiquette mentionnant " Gratidia insolita», noté « Redt.» par erreur. Ce type d'étiquette ancienne ne correspond pas à celles de Brunner et de Redtenbacher, ni par la forme ni par l'écriture habituelle de ces deux auteurs. Par ailleurs, la date de récolte " 1905 », nous paraît trop récente par rapport à la description. En effet, il semble que Brunner et Redtenbacher aient emprunté les spécimens bien avant 1900. Ce spécimen n'est pas non plus noté du Congo. Enfin, la description ne correspond ni par la taille, ni par le fait que le spécimen de Brunner avait les membres postérieurs manquants, ce qui n'est pas le cas de ce spécimen. L'autre spécimen O (MNHN-EO-PHAS1207) placé à ses côtés possède le même genre d'étiquettes, à l'exception de celle marquée du nom d'espèce, et ne correspond pas non plus à la description de Brunner von Wattenwyl (1907).

\section{insularis Lucas, 1869 [Eurycantha]}

Eurycantha insularis Lucas, 1869: 25.

STATUT ACTUEL. - Eurycantha insularis Lucas, 1869.

MatéRIEL TYPE AU MNHN. - 1 ơ ST, 1 \& ST.

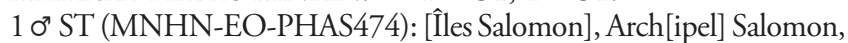
San Georges [San Jorge], H. Lucas, 3-[18]79, 4 [18]79, 165, 165. 1 ㅇ ST (MNHN-EO-PHAS475) : [Îles Salomon], Arch[ipe]l Salomon, San Georges [San Jorge], H. Lucas, 3-[18]79.

\section{REMARQUES}

En 1869, Lucas a décrit le ơ d'Eurycantha calcarata, ainsi que le $0^{\prime \prime}$ et la 9 d'Eurycantha insularis. Mais peu après (Lucas 1872), le même auteur a estimé que ces deux espèces n'en formaient qu'une et a placé $E$. insularis en synonymie avec $E$. calcarata, considérant que le type $\sigma^{7}$ d'E. insularis n'était qu'une "larve» d'E. calcarata. Or ce dernier spécimen présente des terminalia entièrement développés et se trouve donc au stade adulte. La synonymie de Lucas en 1872 est donc injustifiée, ainsi que le déclare Redtenbacher (1908: 345). Sur l'étiquette des spécimens, «1879» représente la date d'entrée au MNHN.

\section{integer Redtenbacher, 1908 [Perisceles]}

Perisceles integer Redtenbacher, 1908: 481.

StATUT ACTUEL. - Abrosoma integrum (Redtenbacher, 1908) Brock \& Seow-Choen 1995: 27 [Abrosoma integer (sic)].

Matériel TYPe AU MNHN. - 4 9 ST.

1 \& ST (MNHN-EO-PHAS765) : [Inde], Mission Maduré, Shembaganor [Shembaganur], $\mathrm{N}^{\circ}$ 31, [collection Pantel].

2 O ST (MNHN-EO-PHAS766, MNHN-EO-PHAS767 associé à un œuf MNHN-EO-PHAS768) : [Inde], Mission Maduré, [collection Pantel].

1 \& ST (MNHN-EO-PHAS769) : N.28 [collection Pantel].
REMARQUE

Le ơ (MNHN-EO-PHAS770) restant dans la collection Pantel ne peut être un ST car Redtenbacher (1908) n'a décrit que la \% de cette espèce. Le nom de genre étant neutre, "integrum» est la forme correcte de l'épithète spécifique.

intermedia Redtenbacher, 1908 [Achrioptera]

Achrioptera intermedia Redtenbacher, 1908: 440.

STATUT ACTUEL. - Achrioptera punctipes punctipes (Audinet-Serville, 1838) - Hennemann \& Conle 2004: 30 (synonymie).

Matériel TYPe au MNHN. - HT o7.

HT ơ (MNHN-EO-PHAS88): Madagascar, Route Laborde, entre Andrangavolo et Ambahanahira, forêt, G. Grandidier, 1899, 26 Janv[ier] [18]99.

\section{intermedia Shelford, 1913 [Libethra]}

Libethra intermedia Shelford, 1913: 61.

STATUT ACTUEL. - Nanolibethra intermedia (Shelford, 1913) Conle et al. 2011: 263.

MatéRIEL TYPe AU MNHN. $-80^{7}$ ST, 12 \% ST. $60^{7}$ ST (MNHN-EO-PHAS602, MNHN-EO-PHAS603, MNHNEO-PHAS604, MNHN-EO-PHAS605, MNHN-EO-PHAS606, MNHN-EO-PHAS607), 11 \% ST (MNHN-EO-PHAS611, MNHNEO-PHAS612, MNHN-EO-PHAS613, MNHN-EO-PHAS614, MNHN-EO-PHAS615, MNHN-EO-PHAS616, MNHN-EOPHAS617, MNHN-EO-PHAS618, MNHN-EO-PHAS619, MNHN-EO-PHAS620, MNHN-EO-PHAS621) (dont 8 juvéniles): Équateur, Casitagua, 3500 m. d'alt., P. Rivet, 1903.

1 ơ ST (MNHN-EO-PHAS608) : Équateur, Quinoaloma, 4000 m. d'alt., P. Rivet, avril, 1904.

1 ơ ST (MNHN-EO-PHAS609), 1 ㅇ ST (MNHN-EO-PHAS622): Équateur, Riobamba, Dr G. Rivet, 1901.

\section{REMARQUE}

Shelford (1913) cite une grande série, mais sans préciser le nombre de spécimens. Il existe 2 autres $\sigma^{7}$ dont le lieu de capture n'est pas cité par Shelford (1913): $10^{7}$ (MNHNEO-PHAS610) : Équateur, Tioloma, 4300 m. d'alt., P. Rivet, mars, 1904; $10^{\circ}$ (MNHN-EO-PHAS623) : Équateur, Pinillar (2900 m), P. Rivet, 1903. 1 ơ ST à l'ANSP (Brock et al. 2018).

\section{intermedia Redtenbacher, 1906 [Orobia]}

Orobia intermedia Redtenbacher, 1906: 71.

STATUT ACTUEL. - Leiophasma intermedium (Redtenbacher, 1906) -Zompro 2004: 247 [Leiophasma intermedia (Redtenbacher, 1906) (sic)]

MATÉRIEL TYPE AU MNHN. -2 9 ST.

1 \& ST (MNHN-EO-PHAS1030), 1 \& ST juvénile (MNHN-EOPHAS1029): Madagascar, M[onta]gne d'Ambre, Ch. Alluaud, 4-[18]96. 


\section{REMARQUE}

Le nom de genre est neutre, l'épithète spécifique doit donc être accordé en conséquence (accord rectifié).

interruptelineatus Brunner von Wattenwyl, 1907

\section{[Clitarchus]}

Clitarchus interruptelineatus Brunner von Wattenwyl, 1907: 236, pl. 10, fig. 4a-d.

STATUT ACTUEL. - Clitarchus hookeri (White, 1846) - Brock 1997: 22 (synonymie).

MatéRIel TYPe au MNHN. - 1 PLT ơ.

1 PLT ơ (MNHN-EO-PHAS454): Nouv[elle]-Zélande, Otago, H. Jacquinot, 1841, 770 [18], 48.

\section{REMARQUE}

LT $0^{x}$ et 2 ㅇ PLT au NHMW; $10^{7}$ PLT au ZMH; 2 ㅇ PLT au ZMB (non répertoriées par Brunner von Wattenwyl (1907)) (Brock 1998a ; Zompro 2002b). Le LT a été désigné par Jewell \& Brock (2003: 195).

\section{inversus Brunner von Wattenwyl, 1907 [Eucarcharus]}

Eucarcharus inversus Brunner von Wattenwyl, 1907: 186, pl. 8, fig. 1.

STATUT ACtUEl. - Tirachoidea inversa (Brunner von Wattenwyl, 1907) - Hennemann \& Conle 2008: 236.

MATÉRIEL TYPE AU MNHN. - HT 9.

HT 9 (MNHN-EO-PHAS325) : [Indonésie], Java Ouest, Tougou, J. D. Pasteur, 3-[18]96, $\mathrm{N}^{\circ}$ 6, 6, 6.

irregularis Redtenbacher, 1906 [Pseudoleosthenes]

Pseudoleosthenes irregularis Redtenbacher, 1906: 160.

STATUT ACTUEL. - Pseudoleosthenes irregularis Redtenbacher, 1906.

MATÉRIEL TYPE AU MNHN. - 1 \% ST.

1 ST (MNHN-EO-PHAS1031) : Madagascar, Isaka, Ch. Alluaud, 1901.

\section{REMARQUE}

La flûte qui contient le spécimen MNHN-EO-PHAS1031 renferme également une + subadulte Parectatosoma sp. 1 \& ST au NHMW (Brock 1998a).

irregulariterdentata Brunner von Wattenwyl, 1907 [Cuniculina]

Cuniculina irregulariterdentata Brunner von Wattenwyl, 1907: 202.

STATUT ACTUEL. - Ramulus mikado (Rehn, 1904) - Ichikawa 2016: 189 (synonymie).

MATÉRIEL TYPE AU MNHN. - HT 9.

HT ㅇ (MNHN-EO-PHAS415): Japon, 1869, 1272 [18]69, 46. javanense Zompro, 2004 [Planispectrum]

Planispectrum javanense Zompro, 2004: 237.

StatuT ACtUel. - Planispectrum javanense Zompro, 2004.

Matériel TYPE AU MNHN. - HT 9 .

HT \& (MNHN-EO-PHAS345) : [Indonésie], Java, J. B. Ledru, R. Oberthür, 1898.

\section{REMARQUE}

Ce spécimen est également un PLT de Spinohirasea bengalensis (Brunner von Wattenwyl, 1907).

\section{jeanneli Chopard, 1938 [Gratidia]}

Gratidia jeanneli Chopard, 1938: 117.

STATUT ACtUel. - Clonaria jeanneli (Chopard, 1938) - Otte \& Brock 2005: 102.

Matériel type au MNHN. - HT ơ.

HT ơ (MNHN-EO-PHAS19) : Kenya, Mission de l'Omo, Camp I de l'Elgon, Mont Elgon, Vers[an]t Est, 2100 m. [2210 m suivant Chopard (1938)], C. Arambourg, P.-A. Chappuis \& R. Jeannel, 1932-[19]33.

jesuitae Brunner von Wattenwyl, 1907 [Carausius]

Carausius jesuitae Brunner von Wattenwyl, 1907: 274.

STATUT ACTUEL. - Carausius sechellensis (Bolívar, 1895) - Brock \& Delfosse 2005: 52 (synonymie).

MATÉRIEL TYPE AU MNHN. - HT 9.

HT 9 (MNHN-EO-PHAS655): Chine, Canton, [collection Pantel].

\section{REMARQUE}

Cette espèce est endémique des Seychelles et sa présence en Chine apparaît douteuse. Cela semble être une erreur d'étiquetage (Brock \& Delfosse 2005).

\section{juvenilis Brunner von Wattenwyl, 1907 [Carausius]}

Carausius juvenilis Brunner von Wattenwyl, 1907: 268.

Statut ACtUel. - Carausius juvenilis Brunner von Wattenwyl, 1907.

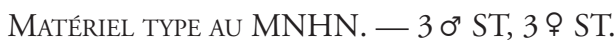

1 ơ ST (MNHN-EO-PHAS694) : [Inde], Shembaganor [Shembaganur], J. Dubreuil, S. J. Mallat, 1901, 32 bis, [collection Pantel]. 1 o ST (MNHN-EO-PHAS699): [Inde], Shembaganor [Shembaganur], J. Dubreuil, 1901, 22 bis, [collection Pantel].

10 ST (MNHN-EO-PHAS695): [Inde], 1, [collection Pantel]. $10^{7}$ ST (MNHN-EO-PHAS696) : [Inde], 32 ter, ơ 9 anth., [collection Pantel].

1 ㅇ ST (MNHN-EO-PHAS697) : [Inde], J. Dubreuil, ㅇ anth., 32, [collection Pantel].

1 ST (MNHN-EO-PHAS698): [Inde], Mission Maduré, [collection Pantel]. 
REMARQUE

1 I ST au NHMW (Brock 1998a; Brock \& Delfosse 2005).
Somacantha kalolohai Cliquennois, 2008: 68, figs 15-22, 53, 56, 57. STATUT ACTUEL. - Somacantha kalolohai Cliquennois, 2008.

Matériel type au MNHN. - HT +2 , $0^{7}$ PT.

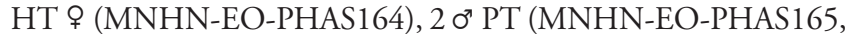
MNHN-EO-PHAS166) : Madagascar, province de Tamatave, Manompana, forêt d'Ambodiriana, 100-200 m, N. Cliquennois 11-19.III.2003.

REMARQUE

2 ơ PT (Coll. NC).

\section{kibonotensis Sjöstedt, 1909 [Gratidia]}

Gratidia kibonotensis Sjöstedt, 1909: 83, fig. 3.

STATUT ACTUEL. - Clonaria kibonotensis (Sjöstedt, 1909) - Otte \& Brock 2005: 102

Matériel TYPe AU MNHN. - 1 ST 9 .

1 ST \& (MNHN-EO-PHAS42): [Tanzanie], Kilimandjaro, Kilimandjaro et Mérou, Expédit[tion] Suédoise, Sjöstedt, Y. Sjösted 12 sept[embre], 1905-[190]6, 1912.

\section{REMARQUE}

Sjöstedt (1909) note 9 ơ $^{\top}$ et 16 ㅇ ST au NHRS mais actuellement seuls 15 spécimens ST sont présents (K. A. Johanson, comm. pers.).

\section{laciniata Redtenbacher, 1906 [Antongilia]}

Antongilia laciniata Redtenbacher, 1906: 25.

Statut aCtUel. - Antongilia laciniata Redtenbacher, 1906

MATÉRIEL TYPE AU MNHN. - $30^{7}$ ST et 4 9 ST.

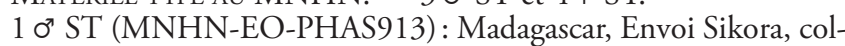
lection Finot.

1 ơ ST (MNHN-EO-PHAS915), 3 9 ST (MNHN-EO-PHAS914, MNHN-EO-PHAS916, MNHN-EO-PHAS1139): Madagascar, collection Finot.

1 ơ ST (MNHN-EO-PHAS103) : Madagascar, G. Grandidier, 1899. 1 ㅇ ST (MNHN-EO-PHAS1138): [collection Finot].

\section{REMARQUE}

Autres ST avérés: 49 et $30^{\prime \prime}$ (NHMW); 1 9 (ZMH) (Cliquennois 2003a; Zompro 2004). Les données «Antongil, Mocqueris leg. » que présente une des $\$$ au NHMWsont erronées, l'espèce vivant en altitude.

\section{laesa Redtenbacher, 1908 [Bacteria]}

Bacteria laesa Redtenbacher, 1908: 419.

STATUT ACtUEl. - Bacteria laesa Redtenbacher, 1908.
Matériel TYPE AU MNHN. - HT \%.

HT \& (MNHN-EO-PHAS575) : Chili, C. Gay, 15-[18]43, 15 [18]43, 188.

\section{laetitiae Bellanger, 2016 [Oncotophasma]}

Oncotophasma laetitiae Bellanger, 2016: 143, figs 1-18.

Statut actuel. — Oncotophasma laetitiae Bellanger, 2016.

Matériel tyPe AU MNHN. - HT ơ, 1 \% PT.

HT ơ (MNHN-EO-PHAS1141), 1 9 PT (MNHN-EO-PHAS1140):

Costa-Rica, Heredia Province, Refugio de Vida Silvestre, Cerro

Dantas, alt. 2000 m, Y. Bellanger, 30.VIII.2011.

\section{laevigatus Brunner von Wattenwyl, 1907 [Clitumnus]}

Clitumnus laevigatus Brunner von Wattenwyl, 1907: 191.

STATUT ACTUEL. - Ramulus ceylonensis Otte \& Brock, 2005: 301 [nom de remplacement pour cause d'homonymie secondaire; $R a$ mulus ceylonense Otte \& Brock, 2005 (sic)].

MATÉRIEL TYPE AU MNHN. - $10^{7}$ ST.

10 ST (MNHN-EO-PHAS1032) : [Inde], Madras, J. Janssen, $87-[18] 72$.

REMARQUE

$40^{7}$ au NHMW ; $10^{7}$ au ZMH ; $10^{7}$ au ZMB ; 1 ou plusieurs ơ au MNMS (Brock 1998a; Zompro 2002b). Le genre Ramulus étant masculin, l'épithète spécifique doit être accordée en conséquence (accord rectifié).

lakini Conle \& Hennemann, 2012 [Pseudophasma]

Pseudophasma lakini Conle \& Hennemann, 2012: 4, figs 1-10.

Statut ACtuel. - Pseudophasma lakini Conle \& Hennemann, 2012.

MATÉRIEL TYPE AU MNHN. $-10^{7}$ PT.

1 Ơ PT (MNHN-EO-PHAS485) : Équateur, Pr. Napo, Baeza / Lago Agrio, San Rafael, C. Amédégnato \& S. Poulain, 02-07.XI.1997, $1300 \mathrm{~m}, 77^{\circ} 35^{\prime} \mathrm{W}, 0^{\circ} 05^{\prime} \mathrm{S}$.

REMARQUE

HT ơ au ZSMC, $20^{7}$ PT et 19 PT au NHMUK, $40^{7}$ PT

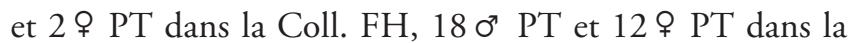
Coll. OC (Conle \& Hennemann 2012).

\section{laminifera Chopard, 1938 [Gratidia]}

Gratidia laminifera Chopard, 1938: 117.

STATUT ACTUEl. - Clonaria laminifera (Chopard, 1938) - Otte \& Brock 2005: 102.

MatéRIEL TYPE AU MNHN. - HT ơ.

HT ơ (MNHN-EO-PHAS30): Kenya, Mission de l'Omo, Mais[on] Forest[ière] Kinangop, M[ont]t Aberdare, Vers[an]t Ouest, 2600 m, C. Arambourg, P.-A. Chappuis \& R. Jeannel, 1932-[19]33. 


\section{REMARQUE}

2 autres $0^{7}$ (MNHN-EO-PHAS31, MNHN-EO-PHAS 32) ne sont pas cités par Chopard (1938), mais font partie de la même série que l'HT.

\section{lelongi Scali, Milani \& Passamonti, 2013 [Pijnackeria]}

Pijnackeria lelongi Scali, Milani \& Passamonti, 2013: 220, fig. 31. StatUT ACTUEL. - Pijnackeria lelongi Scali, Milani \& Passamonti, 2013.

MATÉRIEL TYPE AU MNHN. - HT ơ, 1 \% PT.

HT ơ (MNHN-EO-PHAS1143), 1 ㅇ PT (MNHN-EO-PHAS1142): Esp[agne], Alcocéber, 18.VI.1992.

\section{REMARQUE}

Il s'agit d'une espèce éteinte d'après Scali et al. (2013). Ces deux spécimens sont les seuls connus.

\section{lethargicus Lelong \& Langlois, 1998 [Lamponius]}

Lamponius lethargicus Lelong \& Langlois, 1998: 245, figs 1-9, 22-24. STATUT ACTUEL. — Lamponius lethargicus Lelong \& Langlois, 1998.

MATÉRIEL TYPE AU MNHN. - HT $\$$, AT ơ, 2 ㅇ PT.

HT $९$ (MNHN-EO-PHAS525): Guadeloupe, D11 entre Bains Jaunes et Parking Soufrière, Alt. 950-1100 m, Frédéric Langlois, Philippe Lelong, 01.XI.1995, Esp. nº 10, GUA95006.

AT ơ (MNHN-EO-PHAS526) : Guadeloupe, Trace de la Mamelle du Pigeon, Alt. 600-700 m, Frédéric Langlois, Philippe Lelong, 23 X 95, Esp. n 10, GUA95025.

1 ㅇ PT (MNHN-EO-PHAS528): Guadeloupe, D11 entre Bains Jaunes et Parking Soufrière, Alt. 950-1100 m, Frédéric Langlois, Philippe Lelong, 01.XI.1995, Esp. n 10, GUA95007.

1 \& PT (MNHN-EO-PHAS527) : Guadeloupe, Route de la Soufrière, Alt. 1060 m, Frédéric Langlois, Philippe Lelong, 27 XI 1996, GUA96014.

\section{REMARQUE}

1 ㅇ PT et $10^{7} \mathrm{PT}$ dans la collection PL, qui rejoindront la collection nationale (Lelong \& Langlois 1998).

\section{libanicus Uvarov, 1924 [Bacillus]}

Bacillus libanicus Uvarov, 1924: 8.

Statut aCtuel. - Clonaria libanica (Uvarov, 1924) - Otte \& Brock 2005: 102 .

MATÉRIEL TYPE AU MNHN. - $20^{\circ}$ ST, 2 \% ST.

$10^{7}$ ST (MNHN-EO-PHAS803) : Liban, Bicfaia, P. Torrend, [collection Pantel].

$10^{7}$ ST (MNHN-EO-PHAS805): Liban, n², [collection Pantel]. 1 ơ ST (MNHN-EO-PHAS1179): Syrie, [Mont] Liban, 1887, Collection A. Finot.

1 ㅇ ST (MNHN-EO-PHAS801) et deux de ses œufs (MNHNEO-PHAS802) : [Liban], Torrend, [collection Pantel].

1 o ST (MNHN-EO-PHAS804): Liban, P. Torrend, [collection Pantel]. lineatus Redtenbacher, 1908 [Asceles]

Asceles lineatus Redtenbacher, 1908: 500.

Statut actuel. - Asceles lineatus Redtenbacher, 1908.

MATÉRIEL TYPE AU MNHN. - 1 ST o".

1 ơ ST (MNHN-EO-PHAS380): [Indonésie], Java, R. Oberthür, 97-[18]97, 98.

REMARQUE

1 ㅇ ST au NHMW (Brock 1998a).

\section{lobiventre Blanchard, 1853 [Phyllium]}

Phyllium lobiventre Blanchard, 1853: 359, pl. 1, fig. 9.

STATUT ACTUEL. - Chitoniscus lobiventris (Blanchard, 1853) - Stål 1875: 105.

MATÉRIEL TYPE AU MNHN. - HT o".

HT ơ (MNHN-EO-PHAS398): [Fidji], Iles Viti, Lebouka [Levuka], H. Jacquinot, 1841, 816 [18]41.

\section{lobiventris Chopard, 1952 [Parorobia]}

Parorobia lobiventris Chopard, 1952: 482.

Statut actuel. - Parorobia lobiventris Chopard, 1952.

MATÉRIEL TYPE AU MNHN. - HT ơ

HT ơ (MNHN-EO-PHAS114) : Madagascar, M[on]t Tsaratanana, forêt de bambous à la limite de la forêt de mousses, $2000 \mathrm{~m}$,

R. P[aulian], X.[19]49, Institut scientifique [de] Madagascar.

\section{lobulatipes Pantel, 1917 [Carausius]}

Carausius lobulatipes Pantel, 1917: 274, figs 2, 3.

Statut actuel. - Carausius lobulatipes Pantel, 1917.

MATÉRIEL TYPE AU MNHN. -2 9 ST.

1 ST (MNHN-EO-PHAS676) : [Inde], Shembaganor [Shembaganur], J. Dubreuil, 1901, 26, [collection Pantel].

1 \& ST juvénile (MNHN-EO-PHAS677): [sans étiquette] [collection Pantel].

\section{longeoperculata Redtenbacher, 1908 [Alienobostra]}

Alienobostra longeoperculata Redtenbacher, 1908: 411

STATUT ACTUEL. - Alienobostra jugalis (Rehn, 1905) - Rehn 1905: 72 (synonymie); Otte \& Brock 2005: 43 (nouvelle combinaison).

MATÉRIEL TYPE AU MNHN. - HT 9.

HT + (MNHN-EO-PHAS1033): Guatemala, F. Bocourt, 1512-1882.

\section{longipennis Redtenbacher, 1906 [Anisomorpha]}

Anisomorpha longipennis Redtenbacher, 1906: 92. 
STATUT ACTUEL. - Malacomorpha longipennis (Redtenbacher, 1906) - Zompro 2004: 147.

MATÉRIEL TYPE AU MNHN. - HT 9.

HT \& juvénile (MNHN-EO-PHAS496): Cuba, Mayari, Baie de Nipe, M. Chaper, 1883, 1685 [18]83, 41.

\section{longithorax Redtenbacher, 1908 [Sipyloidea (Rhamphosipyloidea)]}

Sipyloidea (Rhamphosipyloidea) longithorax Redtenbacher, 1908: 550

STATUT ACTUEL. - Rhamphosipyloidea longithorax (Redtenbacher 1908) - Otte \& Brock 2005: 309.

MATÉRIEL TYPE AU MNHN. - HT 9.

HT $~(M N H N-E O-P H A S 356)$ : Philippines, Jalajala, M. Lorquin, 104-[18]60, 98.

macrocercata Delfosse, 2015 [Decidia]

Decidia macrocercata Delfosse, 2015b: 22, figs 1-3.

StatuT ACtUEl. — Decidia macrocercata Delfosse, 2015.

Matériel TyPe AU MNHN. - HT o".

HT ơ (MNHN-EO-PHAS1077) : [Équateur], Ec[uador], Toacaso, Cotopaxi, m. 3300, M. Etonti, 3-XII-2006.

\section{madegassa Redtenbacher, 1906 [Xylica $]$}

Xylica madegassa Redtenbacher, 1906: 30.

STATUT ACTUEL. - Xylica madegassa Redtenbacher, 1906.

MATÉRIEL TYPE AU MNHN. - HT 9.

HT 9 (MNHN-EO-PHAS106) : Madagascar, Région de l'Androy, Ambovombe, Dr J. Decorse, mars [19]01, 1901.

\section{REMARQUE}

Xylica madegassa est un synonyme probable de Paracirsia distincta (Brunner von Wattenwyl, 1907).

maethoraniae Delfosse, 2015 [Phyllium]

Phyllium maethoraniae Delfosse, 2015a: 412, figs 1-5.

Statut actuel. - Phyllium maethoraniae Delfosse, 2015.

MATÉRIEL TYPE AU MNHN. - HT 9.

HT \& (MNHN-EO-PHAS399) : [Thaillande], Thailand, Doi Pui, Chiang Mai.

\section{magdelainei Lucas, 1857 [Phyllium]}

Phyllium magdelainei Lucas, 1857: 147.

STATUT ACTUEL. - Phyllium (Pulchriphyllium) pulchrifolium Audinet-Serville, 1838 - Cumming \& Le Tirant (2018) (synonymie).

MATÉRIEL TYPE AU MNHN. — HT 9.
HT $~(M N H N-E O-P H A S 400)$ : [Indonésie], Sumatra, M. Magdelaine, 395-[18]57, 395 [18]57.

\section{magnifica Hennemann \& Conle, 2004 [Achrioptera]}

Achrioptera magnifica Hennemann \& Conle, 2004: 27, figs 9, 10.

StatuT ACtUel. - Achrioptera magnifica Hennemann \& Conle, 2004.

Matériel tyPe AU MNHN. - HT ơ, 2 ơ PT, 2 9 PT.

HT ơ (MNHN-EO-PHAS120) : Madagascar, Tananarive, collection Le Moult, collection Chulliat.

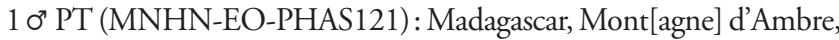
collegit Dr Sicard, [collection Finot].

$10^{7}$ PT (MNHN-EO-PHAS122) : Madagascar, Maroancetra [Maroantsetra], 12.I.1897, collection Finot.

1 ㅇ PT (MNHN-EO-PHAS123): Madagascar, [collection Finot]. 1 \& PT (MNHN-EO-PHAS124) et un œuf(MNHN-EO-PHAS125):

Madagascar, Manjakandriana, Bernard, 1906,

REMARQUE

1 ơ PT au NHMW (Hennemann \& Conle 2004).

magnus Brunner von Wattenwyl, 1907 [Clitarchus]

Clitarchus magnus Brunner von Wattenwyl, 1907: 237.

STATUT ACTUEL. - Baculonistria magna (Brunner von Wattenwyl, 1907) - Bresseel \& Constant 2014: 6.

MATÉRIEL TYPE AU MNHN. - HT $\%$

HT 9 (MNHN-EO-PHAS647) et un œuf (MNHN-EO-PHAS648): [Chine], Chang Hai [Shanghai], P. Joannis, [18]96, [collection Pantel].

magnus Brunner von Wattenwyl, 1907 [Clitumnus]

Clitumnus magnus Brunner von Wattenwyl, 1907: 190.

STATUT ACTUEL. - Ramulus magnus (Brunner von Wattenwyl, 1907) - Otte \& Brock 2005: 304.

MATÉRIEL TYPE AU MNHN. - $10^{7}$ ST.

1 ơ ST (MNHN-EO-PHAS645) : [Indonésie], Java, H. Oberthür, 1898, [collection Pantel].

REMARQUE

2 ơ ST (dont 1 juvénile) au NHMW (Brock 1998a; Brock \& Delfosse 2005).

maindroni Redtenbacher, 1908 [Vetilia]

Vetilia maindroni Redtenbacher, 1908: 464.

STATUT ACTUEL. - Acrophylla maindroni (Redtenbacher, 1908) Günther 1929: 626.

Matériel TYPe au MNHN. - HT 9.

HT \& (MNHN-EO-PHAS1034) : N[ouve]elle-Guinée, Dorey [Manokwari], Raffray \& Maindron, 1878: 88. 
mallati Brunner von Wattenwyl, 1907 [Rhamphophasma] Rhamphophasma mallati Brunner von Wattenwyl, 1907: 210.

StatUT ACTUEL. - Rhamphophasma mallati Brunner von Wattenwyl, 1907.

MatéRIel tYPe AU MNHN. -1 ơ ST, 1 \% ST.

$10^{7}$ ST (MNHN-EO-PHAS703): [Inde], Shembagan[ur], Nº5, [collection Pantel].

1 o ST (MNHN-EO-PHAS704) : [Inde], Shembag[anur], P. Mallat, [collection Pantel].

REMARQUE

3 ST au NHMW (Brock 1998a).

margaritata Redtenbacher, 1908 [Bostra]

Bostra margaritata Redtenbacher, 1908: 412.

StATUT ACtUel. - Bostra margaritata Redtenbacher, 1908.

Matériel TYPe AU MNHN. - HT 9.

HT $\%$ (MNHN-EO-PHAS1035): Mexique, Sierra del Nayarit, L. Diguet, 1898.

marginale Redtenbacher, 1906 [Paraphasma]

Paraphasma marginale Redtenbacher, 1906: 115.

StatuT ACtUel. - Paraphasma marginale Redtenbacher, 1906.

Matériel type Au MNHN. -11 ơ PLT.

$10^{7}$ PLT (MNHN-EO-PHAS531) : Brésil, Roux, 250-[18]36, 250

[18]36, 126.

$10^{7}$ PLT (MNHN-EO-PHAS530) : Brésil, C. Gaudichaud, 1833 , $2679[18] 33$.

$10^{7}$ PLT (MNHN-EO-PHAS1036) : Brésil, Minas Geraes, Caraca,

E. Gounelle, 3113-[18]89.

1 ơ PLT (MNHN-EO-PHAS536) : Brésil, Goyaz, Jatahy, 11.1897, collection Finot.

$20^{7}$ PLT (MNHN-EO-PHAS537, MNHN-EO-PHAS538) : Brésil, Goyaz, Jatahy, 10.1898, [collection Finot].

1 ơ PLT (MNHN-EO-PHAS539): Brésil, Goyaz, Jatahy, collection Finot.

$40^{\prime \prime}$ PLT (MNHN-EO-PHAS532, MNHN-EO-PHAS533, MNHNEO-PHAS534, MNHN-EO-PHAS535) : [Brésil], Montagnes des Orgues, Prov[ince]. de Rio [de] Janeiro, Massif sur la Tijuca, 500 à 900 m d'alt., T. R. Wagner, d'octobre 1901 à mars 1902, 1902.

\section{REMARQUE}

$150^{7}$ PLT et 9 ㅇ PLT au NHMW; $20^{7}$ PLT au ZIN; $30^{7}$ PLT au SMNS; $40^{\circ}$ PLT et 19 PLT au ZMH; $10^{\circ}$ PLT au MTD; PLT détruits dans un incendie au HNHM; 1 LT o", 1 ơ PLT et 2 PLT au MSNG (Zompro 2002b, 2003; Hennemann \& Conle 2003; Brock 1998a, 2007).

\section{marginata Redtenbacher, 1908 [Diacanthoidea]}

Diacanthoidea marginata Redtenbacher, 1908: 532, pl. 26, fig. 6. StaTUT ACTUEl. — Diacanthoidea marginata Redtenbacher, 1908.
Matériel type au MNHN. -1 o ST, 1 9 ST.

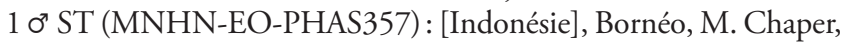
1891, 1293 [18]91, 118.

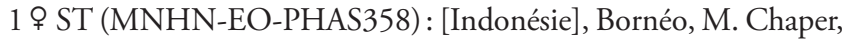
1891, 1293 [18]91, 95.

\section{REMARQUE}

Chaper a exploré la partie occidentale de Bornéo, autour $\mathrm{du}$ fleuve Kapocas (Kapuas), donc actuellement dans la partie indonésienne de l'île (Kalimatan). 4 9 ST et $30^{\circ}$ ST au NHMW; 2 ơ ST au ISNB (Brock 1998a).

\section{marginatus Redtenbacher, 1906 [Presbistus]}

Presbistus marginatus Redtenbacher, 1906: 81.

STATUT ACTUEL. - Kerabistus (Rhadinobistus) marginatus (Redtenbacher, 1906) - Bragg 2001: 292.

Matériel type au MNHN. - 1 PLT ơ.

1 PLT ơ (MNHN-EO-PHAS352): [Indonésie], Bornéo, Lohaban, R. Oberthür, 1898, 71.

\section{REMARQUE}

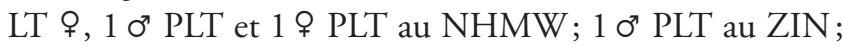
des PLT au MNMS ; 1 \% PLT au ZMH (Brock 1998a; Brock et al. 2018). Le LT a été désigné par Bragg (2001: 292).

\section{maroniensis Chopard, 1911 [Bacteria]}

Bacteria maroniensis Chopard, 1911: 345, fig. [sans numéro].

STATUT ACTUEL. - Bacteria maroniensis Chopard, 1911.

MatéRIel TYPe AU MNHN. — LT $\%, 1$ PLT (juvénile).

LT \& (MNHN-EO-PHAS572) (présente désignation): Guyane franç[ai]se, S[ain]t-Jean du Maroni, Nouveau chantier, Bas-Maroni, collection Le Moult, collection Lucien Chopard 1914.

1 ᄋ PLT juvénile (MNHN-EO-PHAS573): Guyane franç[ai]se, S[ain]t-Jean du Maroni, Juin, collection Le Moult.

\section{REMARQUE}

Il nous semble naturel de désigner l'adulte en tant que LT car c'est celui qui est le plus proche de la description de Chopard.

\section{marshallae Cliquennois \& Brock, 2002 [Apterograeffea]}

Apterograeffea marshallae Cliquennois \& Brock, 2002: 390, figs 9-17. STATUT ACTUEL. - Apterograeffea marshallae Cliquennois \& Brock, 2002.

MATÉRIEL TYPE AU MNHN. - 1 9 PT.

1 아 PT (MNHN-EO-PHAS145): Maurice, Ile Ronde, D. Birch, 2.V.2002.

REMARQUE

HT ơ et 2 ㅇ PT au NHMUK (Cliquennois \& Brock 2002). 
martinicensis Lelong \& Langlois, 2005 [Diapherodes]

Diapherodes martinicensis Lelong \& Langlois, 2005: 264, figs 1930, 40-42.

STATUT ACTUEL. — Diapherodes martinicensis Lelong \& Langlois, 2005.

MATÉRIEL TYPE AU MNHN. — HT 9.

HT $\%$ (MNHN-EO-PHAS542) : Martinique, Trace Duclos Nord, Znieff $\mathrm{n}^{\circ}$ 31, F. Langlois, P. Lelong \& D. Rastel, 10/11/[19]98, 350-637 m, MAR98-036, collection ASPER.

\section{massaica Sjöstedt, 1909 [Gratidia]}

Gratidia massaica Sjöstedt, 1909: 80, fig. 1.

StatuT actuel. - Clonaria massaica (Sjöstedt, 1909) - Otte \& Brock 2005: 103.

MatéRIEL TYPe Au MNHN. -10 o ST.

$10^{7}$ ST (MNHN-EO-PHAS37): [Tanzanie], Kilimandjaro et Mérou, Kilimandjaro, Expédit[ion] Suédoise, Sjöstedt, Y. Sjöstedt, sept[embre], 1905-[190]6, 1912.

\section{REMARQUE}

6 o' ST et 1 o ST au NHRS (K. A. Johanson, comm. pers.), un ơ ST semble donc manquer par rapport aux spécimens répertoriés par Sjöstedt (1909): 8 ơ et 1 ㅇ.

\section{matileorum Donskoff, 1988 [Microcanachus]}

Microcanachus matileorum Donskoff, 1988: 57, figs 1-9.

STATUT ACTUEL. - Microcanachus matileorum Donskoff, 1988.

Matériel type au MNHN. - HT ơ, AT 오, 1 아.

HT ơ (MNHN-EO-PHAS460) : Nouvelle-Calédonie, St.: 251, j,

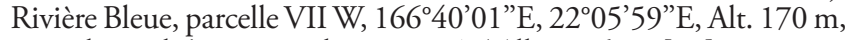
Forêt humide/ pente r.u.b. $25 \mathrm{~m}^{2}$, A. Tillier, 16.VI.[19]87.

AT \& (MNHN-EO-PHAS461): N[ouve]lle-Calédonie, M[on]ts Koghis, 420 m, L. \& D. Matile, 21 XII [19]83.

1 P PT (MNHN-EO-PHAS462) : Nouvelle-Calédonie, St.: 251, h,

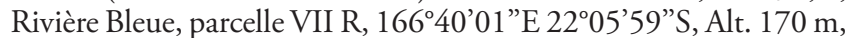
Forêt humide/ pente r.u.b. 25 m, A. \& S. Tillier, 14 Avr[il] [19]87.

\section{maximus Redtenbacher, 1908 [Nearchus]}

Nearchus maximus Redtenbacher, 1908: 448.

STATUT aCtUel. - Phobaeticus magnus Hennemann \& Conle, 2008 - Hennemann \& Conle 2008: 162 (nom de remplacement pour cause d'homonymie secondaire).

MatéRIEL TYPE AU MNHN. -2 9 ST.

2 ㅇ ST (MNHN-EO-PHAS1037, MNHN-EO-PHAS1038) : [Laos], Annam, Laos central, M[on]t[agne]s La Khon [Nakhon Phanom], Harmand, 1877.

\section{REMARQUE}

La localité "Annam» (Vietnam) est en contradiction avec les autres, plus précises, et doit donc être écartée. Considérant que le type (en fait deux ST) de Nearchus maximus Redtenbacher, 1908 était perdu, Hennemann \& Conle (2008) avait désigné un NT o au NHMUK, désignation invalidée par la découverte de ces deux ST qui deviennent automatiquement ceux de Phobaeticus magnus Hennemann \& Conle, 2008.

\section{maxwelli Redtenbacher, 1908 [Bacteria]}

Bacteria maxwelli Redtenbacher, 1908: 420.

STATUT ACTUEL. - Bacteria maxwelli Redtenbacher, 1908 - Büscher \& Baker 2018: 67

MATÉRIEL TYPE AU MNHN. -1 O ST.

ST ơ (MNHN-EO-PHAS571): La Martinique, Rousseau, Dr Rousseau, [18]71, 23 nov[embre] 1871, 23.

REMARQUE

3 ơ ST au NHMW (Brock 1998a; Delfosse 2006). Brock (1998a) considère les 9 du NHMW comme des ST, mais à tort car Redtenbacher (1908) n'a décrit que les ơ.

mayottensis Cliquennois, 2003 [Leiophasma]

Leiophasma mayottensis Cliquennois, 2003b: 415, figs 1-8.

STATUT ACTUEL. - Leiophasma mayottense Cliquennois, 2003.

Matériel tyPe Au MNHN. - HT 9,6 PT, 4 o PT.

HT \& (MNHN-EO-PHAS241): Mayotte, mont Mtsapéré, Majimbini, [alt. 400-500 m], N. Cliquennois, 9.I.2001, NCMY1. $30^{7}$ PT (MNHN-EO-PHAS249, MNHN-EO-PHAS250, MNHNEO-PHAS251), 3 \& PT (MNHN-EO-PHAS246, MNHN-EOPHAS247, MNHN-EO-PHAS248) : Mayotte, mont Mtsapéré, Majimbini, [alt. 400-500 m], N. Cliquennois, 3.I.2002, NCMY20, NCMY21, NCMY22, NCMY15, NCMY17, NCMY18.

1 \& PT (MNHN-EO-PHAS242): Mayotte, mont Mtsapéré, Majimbini, [alt. 400-500 m], N. Cliquennois, 9.I.2001, NCMY3.

1 ơ PT (MNHN-EO-PHAS243): Mayotte, N. Cliquennois, [69].I.2001, NCMY8.

1 우 PT (MNHN-EO-PHAS244): Mayotte, mont Bénara, réserve de Voundzé, [alt. 350 m], N. Cliquennois, 6.I.2001, NCMY12.

1 ㅇ PT (MNHN-EO-PHAS245): Mayotte, mont Bénara, réserve de Voundzé, [alt. 350 m], N. Cliquennois, 6.I.2001, NCMY14.

\section{REMARQUE}

$20^{7}$ PT et 1 ㅇ PT au MHNR; 2 ㅇ PT et 4 ơ PT au MHNG; 2 \% PT et $30^{7}$ PT dans la Coll. PB (Cliquennois 2003a, b). Le nom de genre est neutre, l'épithète spécifique doit donc être accordé en conséquence (accord rectifié).

\section{memorabilis Redtenbacher, 1906 [Pseudodatames]}

Pseudodatames memorabilis Redtenbacher, 1906: 21, pl. 2, figs 5, 6. STATUT ACTUEL. - Pseudodatames memorabilis Redtenbacher, 1906.

Matériel TYPe Au MNHN. - 7 o PLT, 5 o PLT.

$70^{7}$ PLT (MNHN-EO-PHAS839, MNHN-EO-PHAS840, MNHNEO-PHAS842, MNHN-EO-PHAS843, MNHN-EO-PHAS844, MNHN-EO-PHAS845) dont 1 juvénile (MNHN-EO-PHAS846), 5 PLT (MNHN-EO-PHAS841, MNHN-EO-PHAS847, MNHNEO-PHAS848) dont 2 juvéniles (MNHN-EO-PHAS849, MNHNEO-PHAS850): Madagascar, Maroancetra [Maroantsetra], 12.1897, collection Finot. 


\section{REMARQUE}

LT ơ, 2 ơ PLT et 4 ㅇ PLT (dont 1 subadulte) au NHMW; $10^{7}$ PLT et 1 \% PLT au ZMH; $10^{*}$ PLT et 1 \% PLT au ZIN; $10^{7}$ PLT au DEIC; $10^{7}$ PLT à l'ISNB (Cliquennois 2003a; Brock 2007). À noter que deux 9 types de P. memorabilis sont devenues respectivement HT (au ZMH) et PT (au NHMW) de Pseudodatames bicornutus Zompro, 2004. Le LT a été désigné par Zompro (2002a: 7).

\section{millepunctata Redtenbacher, 1906 [Agathemera]}

Agathemera millepunctata Redtenbacher, 1906: 89, pl. 3, fig. 13.

STATUT ACTUEL. - Agathemera millepunctata Redtenbacher, 1906.

MATÉRIEL TYPE AU MNHN. - HT 9 .

HT (MNHN-EO-PHAS643) : [Argentine], Carmen de Pagones, [collection Pantel].

\section{REMARQUE}

Voir Zompro (2004), Brock \& Delfosse (2005), Brock et al. (2018).

\section{minimus Chopard, 1911 [Prisopus]}

Prisopus minimus Chopard, 1911: 342, fig. [sans numéro].

Statut aCtUel. - Prisopus minimus Chopard, 1911.

Matériel TYPe Au MNHN. - HT ơ.

HT ơ (MNHN-EO-PHAS550): Guyane franç[ai]se, Nouveau Chantier, octobre, collection Le Moult, collection Lucien Chopard, 1914, 8.

\section{REMARQUE}

Un autre spécimen ơ de la même espèce (MNHN-EOPHAS551), non pris en compte par Chopard (1911), est présent en collection: Guyane franç[ai]se, S[ain]t Jean du Maroni, collection Le Moult, collection Lucien Chopard, 1914.

\section{minus Chopard, 1919 [Parectatosoma]}

Parectatosoma minus Chopard, 1919: 153.

Statut aCtUel. — Parectatosoma minus Chopard, 1919.

MatérIEL TYPE AU MNHN. - 1 O ST, 1 \% ST.

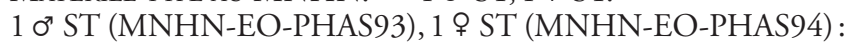
Madagascar, Montagne d'Ambre.

\section{REMARQUE}

Les spécimens portent des étiquettes de LT et de PLT, mais la lectotypification n'a pas été publiée. Ils sont par conséquent toujours des syntypes.

\section{minutissima Redtenbacher, 1906 [Brizoides]}

Brizoides minutissima Redtenbacher, 1906: 113.

STATUT ACtUEL. — Brizoides minutissima Redtenbacher, 1906.

Matériel TYPE AU MNHN. - HT $q$.

HT (MNHN-EO-PHAS529) : [Uruguay], capit[a]le de Montevideo.

\section{REMARQUE}

L'étiquette de détermination indique qu'il s'agit d'un ơ", mais le spécimen est bien une ․

\section{mocquerysi Finot, 1898 [Parectatosoma]}

Parectatosoma mocquerysi Finot, 1898: 585.

Statut actuel. — Parectatosoma mocquerysi Finot, 1898.

MATÉRIEL TYPE AU MNHN. — LT ơ, 11 ơ PLT, 14 9 PLT (présente désignation).

LT O' (MNHN-EO-PHAS863), 11 ơ PLT dont 2 juvéniles (MNHNEO-PHAS95, MNHN-EO-PHAS96, MNHN-EO-PHAS97, MNHN-EO-PHAS854, MNHN-EO-PHAS855, MNHN-EOPHAS856, MNHN-EO-PHAS857, MNHN-EO-PHAS858, MNHN-EO-PHAS859, MNHN-EO-PHAS852, MNHN-EOPHAS853), 14 O ST dont 6 juvéniles (MNHN-EO-PHAS860, MNHN-EO-PHAS861, MNHN-EO-PHAS862, MNHN-EOPHAS866, MNHN-EO-PHAS869, MNHN-EO-PHAS871, MNHN-EO-PHAS872, MNHN-EO-PHAS873, MNHN-EOPHAS851, MNHN-EO-PHAS874, MNHN-EO-PHAS875, MNHN-EO-PHAS876, MNHN-EO-PHAS877, MNHN-EOPHAS878) : Madagascar, Maroancetra [Maroantsetra], 12.1897, collection Finot.

\section{REMARQUES}

Finot (1898) précise le nombre de spécimens qu'il a à sa disposition pour décrire cette espèce: " $90^{*}, 1$ \% peut-être non parvenue à l'état adulte, 10 larves ", tous de la même origine (récolte de Mocquerys en décembre 1897 près de Maroantsetra, Madagascar). Néanmoins, il s'avère difficile de déterminer précisément les spécimens dont il s'est servi dans sa tâche de description. D'abord, sa propre collection ne renferme actuellement que treize spécimens ( $70^{7}$ et 3 ㅇ adultes, ainsi que $20^{\top}$ et 1 \% juvéniles) étiquetés comme appartenant à cette espèce, auxquels on peut ajouter $40^{7}$ adultes arborant les mêmes étiquettes de la collection Finot, 3 dans la collection générale du MNHN et 1 dernier $\sigma^{*}$ au ANSP, ensemble qui apparaît bien apparié et homogène. Or, en contradiction avec ce qu'indique Finot, aucun doute n'est possible quant à la qualité d'adultes de 3 des 4 9 présentes, lesquelles possèdent des ailes courtes, mais parfaitement développées. La lecture de la description vient donner une ébauche d'explication à ce qui apparaît comme une anomalie: ainsi que Carl (1913) le fait remarquer, la description du spécimen $\$$ semble correspondre à une $\$$ du genre Anisacantha, notamment la mention concernant l'absence d'élytres et celle concernant l'allongement du tergum 10 et du sternum 8. Or, Finot avait reçu de Mocquerys, de la même localité (Maroantsetra, sur la baie d'Antongil) des 
spécimens dont Redtenbacher (1906) se servira peu après pour décrire le genre Anisacantha. Il apparaît dès lors que Finot a commis une erreur d'appariement en décrivant sous le même nom des $\sigma^{7}$ et au moins une $\$$ d'espèces distinctes. Sa collection compte par ailleurs 12 \% d'Anisacantha difformis Redtenbacher, 1906, dont 6 juvéniles, spécimens qui correspondent probablement à son matériel d'étude lors de la description de Parectatosoma mocquerysi. Un autre élément, un article de Clément (1903), semble venir indiquer qu'alors Finot s'était rendu compte de sa méprise. En effet, Clément y présente Parectatosoma mocquerysi en donnant une illustration des deux sexes à partir de spécimens que lui a confiés Finot lui-même; or, la représentée ne correspond pas à une $\$$ Anisacantha, mais bien à une + Parectatosoma. Tout bien considéré, il apparaît que doivent être tenus pour des types de Parectatosoma mocquerysi l'ensemble des ơ déterminés comme tels sur une étiquette caractéristique de la collection Finot, soit 12 spécimens dont 2 juvéniles, ainsi que, probablement, les O déterminées "Anisacantha difformis" dans la collection Finot, soit 10 spécimens (MNHN-EO-PHAS866, PHAS869, PHAS871, PHAS872, PHAS873, PHAS874, PHAS8745, PHAS876, PHAS877, PHAS878) également ST d'Anisacantha difformis Redtenbacher, 1906. Cela ne correspond pas exactement aux chiffres avancés par Finot, mais il apparaît vain de vouloir tâcher de résoudre cette différence. De plus, face à une situation si embrouillée, il apparaît justifié de désigner un $0^{7}$ comme LT de l'espèce. 1 ơ PLT à l'ANSP (Cliquennois 2003a; Brock et al. 2018).

\section{modesta Redtenbacher, 1908 [Diardia]}

Diardia modesta Redtenbacher, 1908: 485.

STATUT ACTUEL. - Leprocaulus obiensis (Rehn, 1904) - Rehn 1904: 46 - Günther 1935a: 83 (synonymie).

Matériel TYPe aU MNHN. - 10 O ST.

1 ơ ST (MNHN-EO-PHAS427): [Indonésie], Nouv[elle]-Guinée, Dorey [Manokwari], Raffray \& Maindron, 1878, 2732 [18]78, 102.

\section{REMARQUE}

1 ơ ST au MNMS (Brock et al. 2018).

\section{modesta Brunner von Wattenwyl, 1907 [Dubreuilia]}

Dubreuilia modesta Brunner von Wattenwyl, 1907: 209.

STATUT ACTUEL. - Ramulus neomodestus Otte \& Brock, 2005 - Otte \& Brock 2005: 305 (nom de remplacement pour cause d'homonymie secondaire).

MatéRIEL TYPE AU MNHN. - 1 ơ ST, 1 \& ST.

1 ơ ST (MNHN-EO-PHAS705): [Inde], Shembaganor [Shembaganur], P. Dubreuil, [collection Pantel].

1 ㅇ ST (MNHN-EO-PHAS706) : [Inde], Shembagonor [Shembaganur], $\mathrm{N}^{\circ} 29$, in copula, [collection Pantel]. modestum Redtenbacher, 1906 [Abrosoma]

Abrosoma modestum Redtenbacher, 1906: 85.

STATUT ACTUEL. - Abrosoma modestum Redtenbacher, 1906.

Matériel TYPE AU MNHN. - 1 ST o $0^{*}, 3$ ST 9.

1 Ơ ST (MNHN-EO-PHAS718): [Inde], Shembag[anur], P. Décoly, [collection Pantel].

1 o ST (MNHN-EO-PHAS720): [Inde], Ind[es] Or[ientales], P. Castets, [collection Pantel].

1 \& ST (MNHN-EO-PHAS721): [Inde], Shembaganor [Shembaganur], J. Dubreuil, 1901, [collection Pantel].

1 o ST (MNHN-EO-PHAS719) (juvénile): [sans étiquette] [collection Pantel].

\section{REMARQUE}

Redtenbacher (1906) indique "Trichinopoli, O. Ind." pour les spécimens de la collection Pantel, apparemment par approximation géographique. 3 o $^{7}$ et 2 \% ST au MSNG; 1 ou plusieurs ST au MNMS (Brock \& Delfosse 2005).

montana Brunner von Wattenwyl, 1907 [Gratidia]

Gratidia montana Brunner von Wattenwyl, 1907: 223.

STATUT ACTUEL. - Clonaria montana (Brunner von Wattenwyl, 1907) - Otte \& Brock 2005: 103.

MatéRIEL TYPE AU MNHN. - 2 9 ST, 3 ơ ST.

1 ㅇ ST (MNHN-EO-PHAS50), 1 ㅇ ST (juvénile) (MNHN-EOPHAS54), 3 o" ST (juvéniles) (MNHN-EO-PHAS51, MNHN-EOPHAS52, MNHN-EO-PHAS53): [Éthiopie], Abyssinie, Mission de Bonchamps, Ch. Michel \& M. Potter, 1899.

REMARQUE

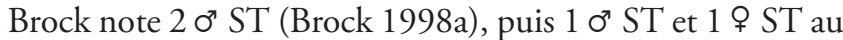
NHMW (Brock et al. 2018).

\section{morio Redtenbacher, 1908 [Sipyloidea]}

Sipyloidea morio Redtenbacher, 1908: 544.

STATUT ACTUEL. — Sipyloidea morio Redtenbacher, 1908.

MATÉRIEL TYPE AU MNHN. $-10^{7}$ ST.

$10^{7}$ ST (MNHN-EO-PHAS353): [Philippines], Manille, 246[18]61, 246 [18]61, 105.

REMARQUE

1 ơ ST au NHMW (Brock 1998a).

\section{morosus Brunner von Wattenwyl, 1907 [Carausius]}

Carausius morosus Brunner von Wattenwyl, 1907: 268.

StatUT ACtUEL. — Carausius morosus Brunner von Wattenwyl, 1907.

Matériel TYPE AU MNHN. - $20^{7}$ ST, 3 \% ST.

$10^{7}$ ST (MNHN-EO-PHAS671): [Inde], Shembagonor [Shembagonur], J. Dubreuil, 1901, ơ anth, 37, [collection Pantel]. 


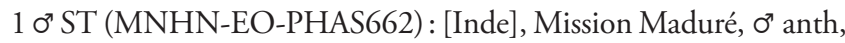
[collection Pantel].

1 P ST et 3 de ses œufs (MNHN-EO-PHAS673) : [Inde], Décoly, [18]97, [collection Pantel].

1 o ST (MNHN-EO-PHAS672) : [Inde], Shembag[onur], J. Dubreuil, 1901, + , anth, 37, [collection Pantel].

1 o ST et ses nombreux œufs (MNHN-EO-PHAS670): [Inde], Kody [= Kodikanel], [collection Pantel].

1 ㅇ ST (MNHN-EO-PHAS669): [Inde], Dixippus morosus Br., [collection Pantel].

\section{REMARQUES}

Lorsque de Sinéty (1901: 121) évoque l'espèce Dixippus morosus, il lui adjoint les mentions «Br. (in litt.)» et précise quelques lignes plus loin: "La plupart de nos espèces exotiques sont inédites; leur description paraîtra dans la grande monographie que M. Brunner de Wattenwyl prépare depuis de longues années. » Cela correspond sans ambiguité au cas mentionné à l'article 8.3 du Code de nomenclature zoologique ( Dénégation de la disponibilité de noms ou actes nomenclaturaux»), qui stipule qu'auquel cas les noms mentionnés ne sont pas disponibles. Dès lors, Sinéty ne saurait être considéré comme l'auteur de cette espèce, et ce même en dépit d'une tradition qui, si ancienne qu'elle soit, demeure mal fondée. Il convient donc de l'attribuer à Brunner von Wattenwyl (1907) qui l'a publiée avec le binom Carausius morosus, c'est-à-dire le binom qui se trouve être en usage actuellement. D'autres ST devraient normalement se trouver au NHMW (Brunner von Wattenwyl 1907). Certains spécimens de la collection Pantel ne présentent aucune donnée qui permettrait de les identifier sûrement comme étant des ST (MNHN-EO-PHAS666, MNHNEO-PHAS667, MNHN-EO-PHAS668, MNHN-EOPHAS674, MNHN-EO-PHAS675, MNHN-EO-PHAS678, MNHN-EO-PHAS679), d'autres sont des individus issus d'élevage (MNHN-EO-PHAS663, MNHN-EO-PHAS664, MNHN-EO-PHAS665).

motalai Cliquennois \& Brock, 2004 [Mauritiophasma]

Mauritiophasma motalai Cliquennois \& Brock, 2004b: 3, figs 1-8, 33.

STATUT ACTUEL. - Mauritiophasma motalai Cliquennois \& Brock, 2004.

MATÉRIEL TYPE AU MNHN. - HT ơ, 2 \% PT.

HT ơ (MNHN-EO-PHAS159): Maurice, Brise Fer-Mare Longue, [500 m], N. Cliquennois, 10-16.III.2001, NCMR27.

2 ㅇ PT (MNHN-EO-PHAS160, MNHN-EO-PHAS161): Maurice, Brise Fer-Mare Longue, [500 m], N. Cliquennois, 10-16.III.2001, NCMR10, NCMR52.

\section{REMARQUE}

1 ơ PT et 2 아 PT au BMHN; 1 아 PT et 1 ơ PT au MHNR; 3 ㅇ PT et $10^{7} \mathrm{PT}$ dans la Coll. PB; 1 \% PT et $10^{7} \mathrm{PT}$ dans la Coll. SM (Cliquennois \& Brock 2004b). multilobata Chopard, 1952 [Cirsia]

Cirsia multilobata Chopard, 1952: 476.

Statut aCtuel. - Cirsia multilobata Chopard, 1952.

MatéRIEL TYPE AU MNHN. - HT $\%$.

HT ? (MNHN-EO-PHAS107) : Madagascar, M[on]t Tsaratanana, forêt de mousses, 1500 m, R. P[aulian], X.[19]49, Institut scientifique [de] Madagascar.

\section{multispinosum Cliquennois \& Brock, 2004 [Heterophasma]}

Heterophasma multispinosum Cliquennois \& Brock, 2004a: 44, figs 1-9.

STATUT ACTUEl. - Heterophasma multispinosum Cliquennois \& Brock, 2004.

Matériel type Au MNHN. - HT \& , 4 ơ PT, 3 \% PT.

HT o (MNHN-EO-PHAS133): [La] Réunion, Bel-Air, SaintJoseph, 1100 m, N. Cliquennois, 22.i.2001, NCR42.

$10^{7}$ PT (MNHN-EO-PHAS134) : La Réunion, Saint-Joseph, BelAir, 1100 m, N. Cliquennois, 22.I.2001, NCR51.

1 o PT (MNHN-EO-PHAS136) : La Réunion, Saint-Joseph, BelAir, 1100 m, N. Cliquennois, 17.I.2002, NCR94.

1 o PT (MNHN-EO-PHAS138): La Réunion, Saint-Joseph, BelAir, 1100 m, N. Cliquennois, 12.I.2002, NCR138.

1 ơ PT (subadulte) (MNHN-EO-PHAS135): La Réunion, forêt du Tévelave, 1100 m, N. Cliquennois, 31.XII.2001, NCR74.

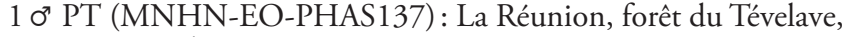
1000 m, N. Cliquennois, 2.II.2002, NCR97.

\section{REMARQUE}

$10^{7}$ et 2 P PT au NHMUK; $10^{*}$ et 2 PT au MHNG; $30^{7}$ et 4 우 PT au MHNR; $10^{7}$ et 1 우 PT dans la Coll. PB. Les spécimens de la forme naine (MNHN-EO-PHAS139, MNHN-EO-PHAS140) ne sont pas des types (Cliquennois \& Brock 2004a).

muricata Redtenbacher, 1906 [Antongilia]

Antongilia muricata Redtenbacher, 1906: 28.

STATUT ACTUEl. - Antongilia muricata Redtenbacher, 1906.

MATÉRIEL TYPE AU MNHN. - 4 ơ ST, 3 ST.

4 ơ ST (MNHN-EO-PHAS930, MNHN-EO-PHAS931, MNHN-

EO-PHAS932, MNHN-EO-PHAS933), 3 \& ST (MNHN-EOPHAS934, MNHN-EO-PHAS935, MNHN-EO-PHAS936) : Madagascar, collection Finot.

REMARQUE

5 ơ ST au NHMW; 1 ㅇ ST au ZIN (Cliquennois 2003a; Brock 2007; Brock et al. 2018).

\section{nairobiensis Bolívar, 1919 [Gratidia]}

nairobiensis Bolívar, 1919: 241 [Gratidia]: 241.

STATUT ACTUEL. - Clonaria nairobiensis (Bolívar, 1919) - Otte \& Brock 2005: 103. 
MATÉRIEL TYPE AU MNHN. - HT ơ.

HT ơ (MNHN-EO-PHAS79): [Kenya], Afrique Orient[ale] Angl[aise], Nairobi, Maurice de Rothschild, Mars, 1906.

\section{naivashensis Bolívar, 1922 [Gratidia]}

Gratidia naivashensis Bolívar, 1922: 206.

STATUT ACTUEL. - Clonaria naivashensis (Bolívar, 1922) - Otte \& Brock 2005: 103.

Matériel TYPe Au MNHN. - HT ơ.

HT ơ (MNHN-EO-PHAS80): [Kenya], Afrique Orient[ale] Angl[aise], Nairobi, Maurice de Rothschild, Mars, 1906.

\section{nana Shelford, 1913 [Ocnophila]}

Ocnophila nana Shelford, 1913: 61.

Statut actuel. - Ocnophila nana Shelford, 1913.

MATÉRIEL TYPE AU MNHN. - 1 O ST, 1 \% ST.

1 ơ ST (MNHN-EO-PHAS624) : Équateur, Chillacocha, 3900 m. d'alt., P. Rivet, Février, 1905.

1 ᄋ ST (MNHN-EO-PHAS625): Équateur, Bueran, 3700 m. d'alt., P. Rivet, 1905.

\section{REMARQUE}

Shelford (1913) cite $10^{\text {Th }}$ et $2 \%$ dans sa description. Il manque donc 1 \& ST provenant de Narihuińa.

nigramala Lelong \& Langlois, 1998 [Paraclonistria]

Paraclonistria nigramala Lelong \& Langlois, 1998: 250, figs 16-21. StATUT ACTUEL. — Paraclonistria nigramala Lelong \& Langlois, 1998.

Matériel type Au MNHN. - HT o , AT ơn, 1 PT, 1 ơ PT. HT $९$ (MNHN-EO-PHAS580): Guadeloupe, D11 entre Bains Jaunes et parking Soufrière, Alt. 1000-1100 m, 30 X [19]95, F. Langlois, P. Lelong, Esp n²0, GUA950-84.

AT O' (MNHN-EO-PHAS581): Guadeloupe, Trace de la Mamelle de Petit-Bourg, Alt. 570 m, F. Langlois, P. Lelong, 26 XI 1996, GUA96037.

1 ㅇ PT (MNHN-EO-PHAS582): entre Bains Jaunes et parking Soufrière, Alt. 950-1000 m, F. Langlois, P. Lelong, 28 X [19]95, Esp n²0, GUA95032.

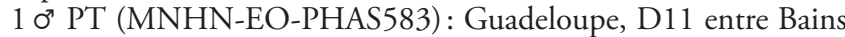
Jaunes et parking de la Soufrière, Alt. 1000-1100 m, F. Langlois, Philippe Lelong, 26 X [19]95, Esp n² 20, GUA95074.

\section{REMARQUE}

107 PT et 1 ㅇ PT dans les Coll. PL et Coll. FL (Lelong \& Langlois 1998).

\section{nigrogranulosus Redtenbacher, 1906 [Perliodes]}

Perliodes nigrogranulosus Redtenbacher, 1906: 137, pl. 5, fig. 8. Statut actuel. - Perliodes nigrogranulosus Redtenbacher, 1906.
MATÉRIEL TYPE AU MNHN. - 3 9 ST.

1 우 ST (MNHN-EO-PHAS1039) (juvénile) : [Panama], I[1]e du Darien, Paya, Vignier, 876-[18]78.

2 ㅇ ST (MNHN-EO-PHAS1040, MNHN-EO-PHAS1041): Brésil, Minas Geraes, E. Gounelle, 3115-[18]85.

REMARQUE

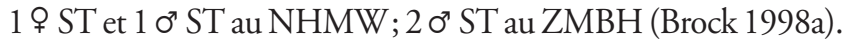

nigrolineata Hennemann, Conle, Bellanger, Lelong \& Jourdan, 2018 [Phantasca]

Phantasca nigrolineata Hennemann, Conle, Bellanger, Lelong \& Jourdan, 2018: 29, figs 49-65.

Statut actuel. - Phantasca nigrolineata Hennemann, Conle, Bellanger, Lelong \& Jourdan, 2018.

Matériel TYPe aU MNHN. - HT ơ, 2 ơ ST, 2 9 ST

HT ơ (MNHN-EO-PHAS1228): Guyane, Saül, [coll. GEP]. $20^{\prime \prime}$ PT (MNHN-EO-PHAS1229, MNHN-EO-PHAS1230) et 1 ㅇ PT (MNHN-EO-PHAS1231): Guyane, Saül, [coll. GEP].

1 우 PT (MNHN-EO-PHAS1224): Guyane fr[ançaise], Saül, Y. Bellanger, T. Jourdan \& P. Lelong, 08-21 X 2013, GUY13-035, coll. ASPER-PL, [coll. GEP].

\section{REMARQUE}

$20^{7} \mathrm{PT}$ et 1 ㅇ PT dans la collection PL, 2 o PT et 1 우 PT dans la collection YB qui seront déposés plus tard au MNHN;

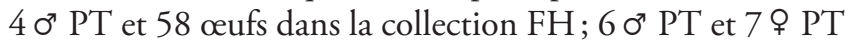
dans la collection OC (Hennemann et al. 2018).

nigrolineata Bresseel \& Constant, 2018 [Pterohirasea]

Pterohirasea nigrolineata Bresseel \& Constant, 2018: 19, figs 9-14.

STATUT ACTUEL. - Pterohirasea nigrolineata Bresseel \& Constant, 2018.

MATÉRIEL TYPE AU MNHN. - $30^{7}$ PT.

3 o' PT (MNHN-EO-PHAS1225, MNHN-EO-PHAS1226, MNHN-EO-PHAS1227): [Vietnam], Annam, Lién Chién près Tourane, 0 à 1000 m. d'alt., Mme Poilane, 1923.

REMARQUE

HT ơ au RBINS, 12 ơ PT et 17 9 PT (dont 2 juvéniles) au RBINS, $50^{7}$ et 7 \% (dont 2 juvéniles) au VNMN (Bressel \& Constant 2018).

nigromaculatus Chopard, 1911 [Prexaspes]

Prexaspes nigromaculatus Chopard, 1911: 340.

STATUt ACtuel. - Prexaspes (Prexaspes) nigromaculatus Chopard, 1911.

MATÉRIEL TYPE AU MNHN. $-20^{\pi}$ ST.

$20^{7}$ ST (MNHN-EO-PHAS500, MNHN-EO-PHAS501): Guyane franç[ai]se, St. Laurent du Maroni, collection Le Moult, collection Lucien Chopard. 
nigrotuberculata Redtenbacher, 1906 [Orobia]

Orobia nigrotuberculata Redtenbacher, 1906: 73.

STATUT ACTUEL. - Leiophasma nigrotuberculatum nigrotuberculatum (Redtenbacher, 1906) - Brock 1998a: 46.

Matériel TYPe Au MNHN. - 6 \% ST, $30^{7}$ ST.

1 ㅇ ST (MNHN-EO-PHAS232): [Madagascar], Imerina, Forêts, Grandidier, 1502-[18]92, 3 Frs., 143.

1 \& ST (MNHN-EO-PHAS233): [Madagascar], Imerina, Forêts, 300 meter [sic] plus bas que mon village, Grandidier, 1502-[18]92, 3 Frs., $144=143$.

$20^{7}$ ST (MNHN-EO-PHAS235, MNHN-EO-PHAS236) : [Madagascar], Imerin, Forêts, Grandidier, 1502-[18]92, Trouvé in copula, 2 Frs.

1 ㅇ ST (MNHN-EO-PHAS234): [Madagascar], 1908, 42, coll[ection] A. Finot.

1 ㅇ ST (MNHN-EO-PHAS237): [Madagascar], 1908, coll[ection] A. Finot.

1 ơ ST (MNHN-EO-PHAS238) : Madagascar, 1908, coll[ection] A. Finot.

2 o ST (MNHN-EO-PHAS882, MNHN-EO-PHAS883) : Madagascar, collection Finot.

REMARQUE

7 ㅇ ST et 8 ơ ST (NHMW); 1 ST (ZIN) (Brock 1998a; Cliquennois 2003a; Brock et al. 2018).

\section{nimbana Chopard, 1955 [Gratidia]}

Gratidia nimbana Chopard, 1955: 99.

STATUT ACtUEL. - Clonaria nimbana (Chopard, 1955) - Otte \& Brock 2005: 104.

MATÉRIEL TYPe AU MNHN. — LT ơ, 1 9 PLT.

LT ơ (MNHN-EO-PHAS33) (présente désignation): Guinée, Nimba, M. Lamotte, II, VI.[19] 42.

1 \& PLT (MNHN-EO-PHAS34) : Guinée, Nimba, 1600 m, M[on] t To, camp I, M. Lamotte, II, VI.[19]42.

\section{REMARQUE}

Chopard (1955) fait état d'un ơ et d'une $\$$ de la même série mais qu'il ne considère pas comme des types: 19 (MNHNEO-PHAS35), idem 9 ST, Guinée, Nimba, 1600 m, Mt To, camp I, M. Lamotte, II, VI.42; 1 o' (MNHN-EO-PHAS36): Guinée, Nimba, M. Lamotte, II, VI.42.

\section{nobilissima Redtenbacher, 1908 [Necroscia]}

Necroscia nobilissima Redtenbacher, 1908: 559.

STATUT ACTUEL. - Laevediacantha zeuxis (Westwood, 1859) Otte \& Brock 2005: 287 (synonymie); Seow-Choen 2016: 113 (nouvelle combinaison).

MatéRIEL TYPe au MNHN. - HT ơ.

HT ơ (MNHN-EO-PHAS338) : [Indonésie], Bornéo, M. Chaper, 1891, 1294 [18]91, 178.

\section{REMARQUE}

Chaper a exploré la partie occidentale de Bornéo, autour du fleuve Kapocas (Kapuas), donc actuellement dans la partie indonésienne de l'île (Kalimatan). Redtenbacher (1908) indique par erreur que ce spécimen est une ㅇ.

$$
\text { nodulosa Redtenbacher, } 1908 \text { [Bacteria] }
$$

Bacteria nodulosa Redtenbacher, 1908: 416.

STATUT ACtUel. - Phanocloidea nodulosa (Redtenbacher, 1908) -Zompro 2001: 196.

MatéRIEL TYPe AU MNHN. - 3 ơ PLT.

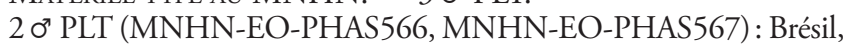

Rivière Lumier, F. Geay, 1899.

1 Ơ $^{7}$ PLT (MNHN-EO-PHAS1042) : Guyane, Ouanary, F. Geay, 1900.

\section{REMARQUE}

LT o $0^{\prime}$ et $10^{\prime \prime}$ PLT au NHMW; $10^{7}$ PLT au SMNS ; 3 PLT au MNMS (Hennemann et al.1995; Brock 1998a; Hennemann \& Conle 2003; Zompro 2006; Brock et al. 2018). Le LT a été désigné par Zompro (2000c: 177).

\section{nodulosus Brunner von Wattenwyl, 1907 [Lonchodes]}

Lonchodes nodulosus Brunner von Wattenwyl, 1907: 261.

STATUT ACTUEL. - Mithrenes whiteheadi (Kirby, 1896) - Kirby 1896: 451 - Hennemann \& Conle 2007: 17 (synonymie).

MATÉRIEL TYPE AU MNHN. - HT o".

HT ơ (MNHN-EO-PHAS633) : [Philippines], Manille, 246[18]61, 246 [18]61, 174.

\section{oberthuri Brunner von Wattenwyl, 1907 [Cuniculina]}

Cuniculina oberthuri Brunner von Wattenwyl, 1907: 200.

STATUT ACTUEL. - Ramulus oberthuri (Brunner von Wattenwyl, 1907) - Otte \& Brock 2005: 305.

Matériel TYPE AU MNHN. - HT $\%$.

HT (MNHN-EO-PHAS419): [Inde], Indes anglaises, Balasore, R. Oberthür, 1898, 3, 3.

obocensis Brunner von Wattenwyl, 1907 [Gratidia]

Gratidia obocensis Brunner von Wattenwyl, 1907: 224.

STATUT ACTUEL. - Clonaria obocensis (Brunner von Wattenwyl, 1907) - Otte \& Brock 2005: 104.

Matériel TYPe AU MNHN. - 4 ơ ST, 10 9 ST et 8 ST juvéniles. 1 ㅇ ST (MNHN-EO-PHAS55): [Somalie], Somalie Angl[aise], Ouadda, F.Jousseaume, 7-[18]97, 166.

1 o ST (MNHN-EO-PHAS56): [Somalie], Somalie Angl[aise], Ouadda, F. Jousseaume, 7-[18]97, 172=166.

1 \& ST (MNHN-EO-PHAS57) (juvénile) : [Somalie], Somalie Angl[aise], Ouadda, F. Jousseaume, 7-[18]97, 169, = 166. 
1 I ST (MNHN-EO-PHAS61): [Somalie], Somalie Angl[aise], Ouadda, F. Jousseaume, 7-[18]97.

1 ST (MNHN-EO-PHAS58) : [Yémen], Aden,F. Jousseaume, 7-[18]97, $168=166$.

1 I ST (MNHN-EO-PHAS60) : [Yémen], Aden, F. Jousseaume, 7-[18]97.

2 ST (MNHN-EO-PHAS59, MNHN-EO-PHAS62) : [Djibouti], Obok,F. Jousseaume, 7-[18]97.

1 ơ ST (MNHN-EO-PHAS64) et 1 \& ST (MNHN-EO-PHAS63) : [Djibouti], Obok, 1885.

3 o ST (dont 1 juvénile) (MNHN-EO-PHAS1043, MNHNEO-PHAS1045, MNHN-EO-PHAS1046), 1 \& ST (MNHNEO-PHAS1044) et 8 juvéniles ST (MNHN-EO-PHAS1047, MNHN-EO-PHAS1048, MNHN-EO-PHAS1049, MNHN-EOPHAS1050, MNHN-EO-PHAS1051, MNHN-EO-PHAS1052, MNHN-EO-PHAS1053, MNHN-EO-PHAS1098) : Djibouti, H. Coutière, 109-[18]97.

\section{REMARQUE}

4 ST et $10^{7}$ ST au NHMW, 1 ST possible au NHMW (Brock 1998a; Brock et al. 2018). Ouadda n'est pas cité par Brunner von Wattenwyl (1907) mais des étiquettes de l'auteur sur les spécimens attestent que cet auteur les a examinés.

\section{olivacea Redtenbacher, 1908 [Monandroptera]}

Monandroptera olivacea Redtenbacher, 1908: 389, pl. 18, fig. 3.

STATUT ACTUEL. - Monandroptera acanthomera (Burmeister, 1838) - Cliquennois \& Brock 2004a: 50 (synonymie).

MATÉRIEL TYPE AU MNHN. - 107 PLT, 4 9 PLT.

$10^{7}$ PLT (MNHN-EO-PHAS141) : La Réunion, E. Bordage, [18]97, 162.

1 o PLT (MNHN-EO-PHAS142) : La Réunion, E. Bordage, [18]97, 161.

2 ९ PLT (MNHN-EO-PHAS143, MNHN-EO-PHAS144) : La Réunion, E. Bordage, [18]97.

1 ㅇ PLT (MNHN-EO-PHAS1054) (juvénile) : Ile Maurice, 1889.

\section{REMARQUE}

LT $0^{\star}, 10^{7}$ PLT et 2 P PLT au NHMW; 1 P PLT au SMNS (Hennemann et al. 1995; Hennemann \& Conle 2003; Cliquennois \& Brock 2004a; Zompro 2006).

\section{olivaceus Chopard, 1911 [Prexaspes]}

Prexaspes olivaceus Chopard, 1911: 339.

Statut aCtuel. - Prexaspes (Prexaspes) olivaceus Chopard, 1911 - Otte \& Brock 2005: 282.

MATÉRIEL TYPE AU MNHN. - HT 9.

HT (MNHN-EO-PHAS503) : Guyane franç[ai]se, S[ain]t-Laurent du Maroni, collection Le Moult, collection Lucien Chopard 191.

\section{REMARQUE}

Une autre \& (MNHN-EO-PHAS502) n'est pas répertoriée par Chopard (1911), mais elle appartient à la même série. operculata Redtenbacher, 1906 [Orobia]

Orobia operculata Redtenbacher, 1906: 70.

STATUT ACTUEL. - Leiophasma operculatum (Redtenbacher, 1906) - Brock 1998a: 48.

MATÉRIEL TYPE AU MNHN. - 7 ㅇ ST, 3 o ST.

7 ㅇ ST (MNHN-EO-PHAS893, MNHN-EO-PHAS895, MNHN-EO-PHAS896, MNHN-EO-PHAS897, MNHN-EOPHAS898, MNHN-EO-PHAS899, MNHN-EO-PHAS900), $30^{7}$ ST (MNHN-EO-PHAS894, MNHN-EO-PHAS901, MNHNEO-PHAS902): Madagascar, Maroancetra [Maroantsetra], 12.1897, collection Finot.

REMARQUE

$20^{7}$ ST et 2 \% ST au NHMW ; 1 \% ST au ZMH; 1 ㅇ ST au ZIN (Brock 1998a, 2007; Zompro 2002b; Cliquennois 2003a).

\section{pallidus Chopard, 1911 [Metriotes]}

Metriotes pallidus Chopard, 1911: 341.

STATUT ACTUEL. - Metriophasma (Metriophasma) pallidum (Chopard, 1911) - Otte \& Brock 2005: 202.

Matériel TYPe au MNHN. - HT 9.

HT $\%$ (MNHN-EO-PHAS498) : Guyane Fr anç[ai]se, S[ain]t-Jean du Maroni, Janvier, collection Le Moult, collection Lucien Chopard.

$$
\text { panteli Redtenbacher, } 1908 \text { [Asceles] }
$$

Asceles panteli Redtenbacher, 1908: 497.

STATUT ACTUEL. - Asceles margaritatus margaritatus Redtenbacher, 1908 - Seow-Choen 2016: 41 (synonymie).

MATÉRIEL TYPE AU MNHN. - HT \%

HT \& (MNHN-EO-PHAS639): [Malaisie], Nord Bornéo, O. Staudinger, 1898, [collection Pantel].

\section{pardalina Redtenbacher, 1906 [Onogastris]}

Onogastris pardalina Redtenbacher, 1906: 24, pl. 2, figs 10, 11.

STATUT ACTUEL. - Onogastris pardalina Redtenbacher, 1906.

MatéRIEL TYPE AU MNHN. - 1 O ST, 1 \& ST.

$10^{7}$ ST (MNHN-EO-PHAS1055) et 1 \% ST (MNHN-EOPHAS1056): Madagascar, Sakavalana, Ch. Alluaud, 1901 in cop[ula].

\section{REMARQUE}

Cliquennois (2009) a proposé un NT avant que le couple de ST soit retrouvé: $10^{\top 7}$ (MNHN-EO-PHAS112): Madagascar, SEE, N. Isaka-Ivondro, 3.VI.1968, D. Wintrebert rec. La découverte des ST destitue naturellement ce spécimen de son statut de type porte-nom (article $75.8 \mathrm{du}$ Code international de Nomenclature zoologique). 


\section{parva Redtenbacher, 1908 [Parasosibia]}

Parasosibia parva Redtenbacher, 1908: 482.

StATUT ACtUel. - Parasosibia parva Redtenbacher, 1908.

MATÉRIEL TYPE AU MNHN. - 5 \% ST.

1 \& ST (MNHN-EO-PHAS771) (juvénile): [Inde], Ind[es] Or[ientales], P. Castets, [collection Pantel].

1 ST (MNHN-EO-PHAS772) : [Inde], Kod[aikanal], Décoly, 1898, [collection Pantel].

1 o ST (MNHN-EO-PHAS1144): [Inde], Kodikanel [Kodaikanal], P. Décoly, 150, [collection Pantel].

1 ST avec œufs et immatures associés (MNHN-EO-PHAS1149): [Inde], Kodikanel [Kodaikanal], Décoly, [18]95, [collection Pantel]. 1 ᄋ ST (MNHN-EO-PHAS1155): [Inde], nº 18, [collection Pantel].

\section{REMARQUES}

10 autres spécimens $\$$ et immatures (MNHN-EO-PHAS1145, MNHN-EO-PHAS 1 146, MNHN-EO-PHAS 1147 , MNHN-EO-PHAS 1148 , MNHN-EO-PHAS 1150 , MNHN-EO-PHAS1151, MNHN-EO-PHAS1152, MNHNEO-PHAS1153, MNHN-EO-PHAS1154, MNHN-EOPHAS1156) sans informations de localité sont présents dans la collection Pantel. Au moins 1 ㅇ ST au MNMS (Brock \& Delfosse 2005; Brock et al. 2018). Redtenbacher (1908) donne "Trichinopoly" comme localité: voir la remarque faite pour Abrosoma modestum.

\section{parvipennis Redtenbacher, 1906 [Xerantherix]}

Xerantherix parvipennis Redtenbacher, 1906: 161, pl. 6, fig. 4.

STATUT ACTUEL. - Xerantherix parvipennis Redtenbacher, 1906.

Matériel TYPE AU MNHN. - 5 \% ST.

5 O ST (MNHN-EO-PHAS904, MNHN-EO-PHAS905, MNHNEO-PHAS906, MNHN-EO-PHAS907, MNHN-EO-PHAS908) : Madagascar, Maroancetra [Maroantsetra], 12.1897, collection Finot.

\section{REMARQUE}

3 ST au NHMW ; 1 ㅇ ST au ZMH (Brock 1998a; Zompro 2002b; Cliquennois 2003a).

\section{pauliani Cliquennois, 2008 [Cenantherix]}

Cenantherix pauliani Cliquennois, 2008: 75, figs 32-34.

Statut actuel. - Cenantherix pauliani Cliquennois, 2008.

MATÉRIEL TYPE AU MNHN. - HT 9

HT $~(M N H N-E O-P H A S 116)$ : Madagascar, M[on]t Tsaratanana, forêt de mousses, 1500 m, R. P[aulian], X.[19]49, Inst. Scient. Madagascar.

\section{pauliani Chopard, 1952 [Onogastris]}

Onogastris pauliani Chopard, 1952: 478.

StatUT ACTUEl. — Onogastris pauliani Chopard, 1952.

MATÉRIEL TYPE AU MNHN. — HT ơ, AT $\%$.
HT ơ (MNHN-EO-PHAS287), AT $~(M N H N-E O-P H A S 288)$ : Madagascar, $\mathrm{M}$ [on]t Tsaratanana, forêt de mousses, 1500-1800 m, R. P[aulian], X.[19]49, Institut scientifique [de] Madagascar.

\section{pavisae Langlois \& Lelong, 1998 [Hesperophasma]}

Hesperophasma pavisae Langlois \& Lelong, 1998: 454, figs 10-12. STATUT ACTUEL. - Hesperophasma pavisae Langlois \& Lelong, 1998. Matériel TYPE AU MNHN. - HT 9 .

HT ㅇ (MNHN-EO-PHAS524): Guadeloupe, Trace V[ictor]Hugues, A. Grodé, [19]68, GUA68001, [coll. INRA].

\section{pentlandi Redtenbacher, 1906 [Autolyca]}

Autolyca pentlandi Redtenbacher, 1906: 95.

STATUT ACTUel. - Peruphasma pentlandi (Redtenbacher, 1906) Conle \& Hennemann 2002: 89.

MATÉRIEL TYPE AU MNHN. - $10^{7}$ PLT, 2 \% PLT.

$10^{7}$ PLT (MNHN-EO-PHAS492), 2 \% PLT (MNHN-EO-PHAS490,

MNHN-EO-PHAS491): Pérou, Cusco, J. B. Pentland, 1839, 255 [18]39.

REMARQUE

LT ơ, 2 \& PLT au NHMW (Brock 1998a).

$$
\text { perplexus Redtenbacher, } 1908 \text { [Asceles] }
$$

Asceles perplexus Redtenbacher, 1908: 499.

STATUT ACTUEL. - Maculonecroscia perplexa (Redtenbacher, 1908) - Seow-Choen 2016: 121.

MATÉRIEL TYPE AU MNHN. - 1 O ST.

$10^{7}$ ST (MNHN-EO-PHAS381) : [Inde], Bengale, Diard \& Duvaucel, 1815,95 .

\section{REMARQUE}

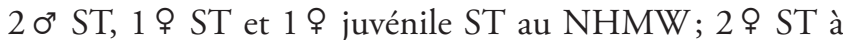
l'ISNB (Brock 1998a; Brock et al. 2018). Le nom de genre étant féminin, l'épithète spécifique doit être accordée en conséquence (accord rectifié).

\section{picturata Redtenbacher, 1906 [Autolyca]}

Autolyca picturata Redtenbacher, 1906: 95.

STATUT ACTUEL. - Autolyca picturata Redtenbacher, 1906.

MATÉRIEL TYPE AU MNHN. - HT 9 .

HT + (MNHN-EO-PHAS493) : Chili ?, 171.

pinnatus Redtenbacher, 1908 [Pericentrus]

Pericentrus pinnatus Redtenbacher, 1908: 352.

Statut actuel. - Pericentrus pinnatus Redtenbacher, 1908. 
MATÉRIEL TYPE AU MNHN. - HT 9 .

HT ㅇ (MNHN-EO-PHAS422): [Inde], Bhoutan, 1898, MariaBasti, M[ission] Durel, R. Oberthür, 1898, 172.6.

\section{pinnipes Redtenbacher, 1908 [Pharnacia]}

Pharnacia pinnipes Redtenbacher, 1908: 452.

STATUT ACTUEL. - Phobaeticus pinnipes (Redtenbacher, 1908)Hennemann \& Conle 2008: 174.

MatéRIEL TYPe AU MNHN. - HT ơ.

HT ơ (MNHN-EO-PHAS1114) : [Malaisie], Malacca, Selangor, Errington et Chapé, 1900.

\section{pinoruminsulae Delfosse, 2013 [Trapezaspis]}

Trapezaspis pinoruminsulae Delfosse, 2013: 17, figs 1-6.

STATUT ACtUel. - Trapezaspis pinoruminsulae Delfosse, 2013.

MATÉRIEL TYPE AU MNHN. - HT 9.

HT (MNHN-EO-PHAS456) : [Nouvelle-Calédonie], Ile des Pins,

Baie d'Oro, Forêt au sol, M. Fromaget, 4 III [19]90.

\section{piperinus Redtenbacher, 1906 [Prisopus]}

Prisopus piperinus Redtenbacher, 1906: 154, pl. 5, fig. 16.

Statut aCtUel. - Prisopus piperinus Redtenbacher, 1906.

MATÉRIEL TYPE AU MNHN. -2 \& ST.

1 \& ST (MNHN-EO-PHAS552): Guyane franç[aise], S[ain]tLaurent, E. Mélinon, 1862, 331 [18]62, 141.

1 ㅇ ST (MNHN-EO-PHAS553) (juvénile): Guyane franç[aise], E. Mélinon, 1863, 255 [18]63, 146.

\section{REMARQUE}

2 오 ST au NHMW (Brock 1998a).

\section{plagiatus Redtenbacher, 1906 [Isagoras}

Isagoras plagiatus Redtenbacher, 1906: 135.

STATUT ACTUEL. - Isagoras plagiatus Redtenbacher, 1906.

MATÉRIEL TYPE AU MNHN. $-20^{*}$ ST.

$10^{7}$ ST (MNHN-EO-PHAS516) : [Colombie], Bogota, 201-[18]62, 201 [18]62, 101a.

1 ơ ST (MNHN-EO-PHAS515) : 56.

\section{REMARQUE}

1 ST et $60^{7}$ ST au NHMW, 2 juvéniles ST au MNMS; $20^{7}$ ST au ZIN; $10^{7}$ ST au NHMUK; 4 o" ST au ZMH; 1 ơ ST au ZMB (Brock 1993, 1998a, 2007; Zompro 2002b).

\section{porrectus Brunner von Wattenwyl, 1907 [Clitumnus]}

Clitumnus porrectus Brunner von Wattenwyl, 1907: 196.
STATUT ACTUEL. - Ramulus artemis (Westwood, 1859) - Westwood, 1859: 10, pl. 26 - Hennemann et al. 2008: 27 (synonymie).

MATÉRIEL TYPE AU MNHN. - LT 9,1 PLT.

LT + (MNHN-EO-PHAS701) : [Inde], Kurseong, [18]98, Décoly, seul spécimen trouvé sur la route [collection Pantel].

1 ○ PLT (MNHN-EO-PHAS702): [Inde], Kurseong, Bengale [collection Pantel].

REMARQUE

Au moins 1 PLT au NZSI (Brock et al. 2018).

$$
\text { postspinosaSjöstedt, } 1909 \text { [Gratidia] }
$$

Gratidia postspinosa Sjöstedt, 1909: 84, fig. 4.

StatuT aCtUel. - Clonaria postspinosa (Sjöstedt, 1909) - Otte \& Brock 2005: 104.

MATÉRIEL TYPE AU MNHN. $-10^{\pi}$ ST.

$10^{7}$ ST (MNHN-EO-PHAS7): [Tanzanie], Kilimandjaro et Mérou, Méru, Nieder, Ngare Na Nyuki, Expédit[ion] Suédoise, Sjöstedt, Y. Sjöstedt, jan[vier], 1905-[190]6, 1912.

REMARQUE

$50^{7}$ ST et 1 9 ST au NHRS (K. A. Johanson comm. pers.), 1 o' ST au ZMHB (Brock et al. 2018). Un ơ ST est manquant car Sjöstedt (1909) notait 8 o' ST et 1 ᄋ ST au total.

\section{poulaini Cliquennois, 2008 [Paranisacantha]}

Paranisacantha poulaini Cliquennois, 2008: 66, figs 7-14.

Statut actuel. - Paranisacantha poulaini Cliquennois, 2008.

MATÉRIEL TYPE AU MNHN. - HT 오 3 오 PT, 10 ㄱ. PT.

HT 9 (MNHN-EO-PHAS172), 1 9 PT (MNHN-EO-PHAS173): Madagascar Est, Marojezy, mission CNRS, R.C.P. N²25, XII.1972. 1 ㅇ PT (MNHN-EO-PHAS174) : Madagascar Est, massif du Marojezy, rés[erve] intégr[ale] nat[urelle] intégr[ale] 12, $1300 \mathrm{~m}$, A. Peyrieras, 12.XII.1972.

1 o PT (MNHN-EO-PHAS175): Madagascar Est, massif du Marojezy, rés[erve] intégr[ale] nat[urelle] intégr[ale] 12, $1300 \mathrm{~m}$, A. Peyrieras, 13/16.XII.1972.

1 ơ PT (MNHN-EO-PHAS176): Madagascar Est, massif du Marojezy, rés[erve] intégr[ale] nat[urelle] intégr[ale] 12, $1300 \mathrm{~m}$, A. Peyrieras, 2/8.XII.1972.

\section{proboscidea Bolívar, 1922 [Gratidia]}

Gratidia proboscidea Bolívar, 1922: 208.

STATUT ACTUEL. - Clonaria proboscidea (Bolívar, 1922) - Otte \& Brock 2005: 104.

Matériel TYPe AU MNHN. - HT o".

HT ơ (MNHN-EO-PHAS72): Éthiopie, Éthiopie Mérid[ionale], Tchorré, Maurice de Rothschild, août, 1905. 
prospera Brunner von Wattenwyl, 1907 [Leptinia]

Leptinia prospera Brunner von Wattenwyl, 1907: 231.

STATUT ACTUEL. - Maransis mozambicus (Westwood, 1859) Westwood 1859: 231 - Otte \& Brock 2005: 191 (synonymie).

Matériel TYPe AU MNHN. - HT ơ.

HT ơ (MNHN-EO-PHAS6) : [Tanzanie], Kondoa, M. Bloyet, $1885,6772[18] 95,84$.

punctipes cliquennoisi Hennemann \& Conle, 2004 [Achrioptera]

Achrioptera punctipes cliquennoisi Hennemann \& Conle, 2004: 34 , figs $7,50,90$.

STATUT ACTUEL. - Achrioptera punctipes cliquennoisi Hennemann \& Conle, 2004.

Matériel type au MNHN. - HT ơ, 1 \% PT.

HT ơ (MNHN-EO-PHAS126) : [Madagascar], province de Tamatave, Manompana, forêt d'Ambodiriana, 100-200 m, N. Cliquennois, 15.III.2003.

1 P PT (MNHN-EO-PHAS127) et ses œufs associés (MNHNEO-PHAS128): [Madagascar], province de Tamatave, Manompana, forêt d'Ambodiriana, 100-200 m, N. Cliquennois, 19.III.2003.

\section{REMARQUE}

1 P PT et ses œufs associés au ZSMC; 6 œufs dans la coll. FH.

\section{pusillus Redtenbacher, 1906 [Datames]}

Datames pusillus Redtenbacher, 1906: 52.

STATUT ACtUel. - Planispectrum pusillum (Redtenbacher, 1906) - Zompro 2004: 238.

MATÉRIEL TYPE AU MNHN. - 1 o ST, 1 \% ST.

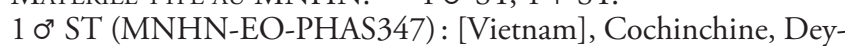
rolle, 1861, 226 [18]61, 146 .

1 ㅇ ST (MNHN-EO-PHAS346) : [Vietnam], Cochinchine, Deyrolle, 1861, 226 [18]61.

\section{pustulosus Pantel, 1917 [Carausius]}

Carausius pustulosus Pantel, 1917: 277, pl. 2, fig. 6.

StATUt ACtUEl. — Carausius pustulosus Pantel, 1917.

MATÉRIEL TYPE AU MNHN. - HT 9.

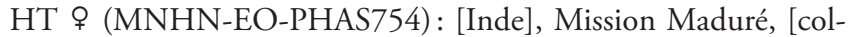
lection Pantel].

\section{quadrilobata Chopard, 1911 [Bacteria]}

Bacteria quadrilobata Chopard, 1911: 343, fig. [sans numéro].

STATUT ACTUEl. - Phantasca quadrilobata (Chopard, 1911) Hennemann et al. 2018: 43.

Matériel type Au MNHN. — LT o , 1 \% PLT.
1 o LT (MNHN-EO-PHAS577): [Guyane française], Cayenne, collection Lucien Chopard 191.

1 o PLT (MNHN-EO-PHAS578): Guyane française, Cayenne, Février, Coll. Le Moult collection Lucien Chopard 191.

REMARQUE

Le LT a été désigné par Hennemann et al. (2018).

quadrisignata Redtenbacher, 1908 [Marmessoidea]

Marmessoidea quadrisignata Redtenbacher, 1908: 512.

STATUT ACTUEL. - Marmessoidea quadrisignata Redtenbacher, 1908.

MatéRIEL TYPE AU MNHN. - HT o".

HT ơ (MNHN-EO-PHAS368): [Indonésie], Java, 9-[18]52, 9 [18]52, 104.

quadrispinosa Redtenbacher, 1906 [Xylica]

Xylica quadrispinosa Redtenbacher, 1906: 29, pl. 3, fig. 5.

STATUT ACTUEL. - Xylica quadrispinosa Redtenbacher, 1906.

Matériel tYPe AU MNHN. - 1 ST $0^{*}$.

1 ST ơ (MNHN-EO-PHAS5): [Tanzanie], Kondoa, M. Bloyet, $1885,6773[18] 85,81$.

\section{REMARQUE}

Redtenbacher (1906) indique deux localités, Zanzibar et Kondoa; celles-ci apparaissant distinctes, cela signifierait qu'il existerait au moins deux ST.

\section{quadrituberculata Chopard, 1952 [Antongilia]}

cf. Antongilia chopardi Cliquennois, 2003.

$$
\text { quinquecarinata Chopard, } 1938 \text { [Gratidia] }
$$

Gratidia quinquecarinata Chopard, 1938: 118.

STATUT ACTUEL. - Clonaria quinquecarinata (Chopard, 1938)Otte \& Brock 2005: 104.

Matériel TYPE AU MNHN. - HT $\%$.

HT + (MNHN-EO-PHAS18) : Kenya, mission de l'Omo, Marakwet, Elgeyo Escarpment, 2500 m, C. Arambourg, P. A. Chappuis \& R. Jeannel, 1932-[19]33.

\section{raffrayi Brunner von Wattenwyl, 1907 [Carausius]}

Carausius raffrayi Brunner von Wattenwyl, 1907: 272.

STATUT ACTUEL. - Leprocaulinus vipera vipera (Kaup, 1871): 39. - Günther 1935a: 82 (synonymie); Otte \& Brock 2005: 173 (nouvelle combinaison).

Matériel type au MNHN. - HT o 
HT ㅇ (MNHN-EO-PHAS1057) : N[ouv]elle-Guinée, Dorey [Manokwari], Raffray et Maindron, 1878.

rectangulata Bolívar, 1922 [Gratidia]

Gratidia rectangulata Bolívar, 1922: 209.

STATUT ACTUEL. - Clonaria rectangulata (Bolívar, 1922) - Otte \& Brock 2005: 104.

MatéRIEL TYPE AU MNHN. $-20^{\circ}$ ST.

2 o $^{7}$ ST (MNHN-EO-PHAS76, MNHN-EO-PHAS77) : [Kenya], Afrique Orient[ale] Angl[aise], Nairobi, Maurice de Rothschild, Août, 1906.

rehni Bolívar, 1922 [Gratidia]

Gratidia rehni Bolívar, 1922: 207.

STATUT ACTUEL. - Clonaria rehni (Bolívar, 1922) - Otte \& Brock 2005: 105.

MATÉRIEL TYPE AU MNHN. - HT ơ.

HT ơ (MNHN-EO-PHAS73) : Éthiopie, Éthiopie Mérid[ionale], Haut Aouache, Endessa, Maurice de Rothschild, Août, 1905.

\section{REMARQUE}

Bolívar indique «ㅇ " par erreur à la suite des mensurations.

\section{retracta Redtenbacher, 1908 [Sosibia]}

Sosibia retracta Redtenbacher, 1908: 537.

STATUT actuel. - Calvisia conicipennis (Bates, 1865) - SeowChoen 2016: 51 (synonymie).

Matériel TYPe AU MNHN. - 1 \% ST.

1 \& ST (MNHN-EO-PHAS370) : [Indonésie], Bornéo, M. Chaper, 1891, 1290 [18]91, 116.6.

\section{REMARQUE}

Chaper a exploré la partie occidentale de Bornéo, autour du fleuve Kapocas (Kapuas), donc actuellement dans la partie indonésienne de l'île (Kalimatan). 1 ơ ST et 1 ㅇ ST au NHMW (Brock 1998a).

\section{reunionensis Cliquennois \& Brock, 2002 [Apterograeffea]}

Apterograeffea reunionensis Cliquennois \& Brock, 2002: 388, figs 1-8.

StatUT ACTUEL. - Apterograeffea reunionensis Cliquennois \& Brock, 2002.

MATÉRIEL TYPE AU MNHN. - HT ơ, 3 ơ PT, 3 o PT,

HT $\sigma^{7}$ (MNHN-EO-PHAS146): La Réunion, Saint-Philippe, [Hauts de Basse-Vallée], La Vallée-Heureuse, 600-700 m, [N. Cliquennois], 8.IX.2001, NCR67.

1 \& PT (MNHN-EO-PHAS150) : Réunion, Saint-Philippe, [Hauts de Basse-Vallée], La Vallée-Heureuse, 600-700 m, [N. Cliquennois], 8.IX.2001, NCR65.
1 ơ PT (MNHN-EO-PHAS151): Réunion, Saint-Philippe, [Hauts de Basse-Vallée], La Vallée-Heureuse, 600-700 m, [N. Cliquennois], 8.IX.2001, NCR68.

1 ơ PT et 1 9 PT in copula (MNHN-EO-PHAS147): La Réunion, Saint-Philippe, [Hauts de] Basse-Vallée, 600 m, [N. Cliquennois], 23.II.2001, RF4bis, f: NCR45, m: NCR46.

1 \& PT (MNHN-EO-PHAS148) : La Réunion, Bras-Panon, sentier de la Plaine des Lianes, 800 m, [N. Cliquennois], 9.VI.2001, NCR57.

1 ơ PT (MNHN-EO-PHAS149) : La Réunion, Bras-Panon, sentier de la Plaine des Lianes, 800 m, [N. Cliquennois], 9.VI.2001, NCR54.

REMARQUE

2 ㅇ PT et $20^{\prime \prime}$ PT au NHMUK; 3 ơ PT et 2 아 PT au MHNG; 3 우 PT et 1 ơ PT au NHMW; 1 우 PT et $20^{7}$ PT au MHNR; 1 ㅇ PT et 1 ơ PT au MSIRI; 1 ㅇ PT et $10^{7}$ PT dans la Coll. PB (Cliquennois \& Brock 2002).

\section{rivalis Redtenbacher, 1908 [Necroscia]}

Necroscia rivalis Redtenbacher, 1908: 562.

StatuT actuel. - Paranecroscia rivalis (Redtenbacher, 1908) Seow-Choen 2016: 214.

MATÉRIEL TYPe AU MNHN. - 1 O' ST, 1 \% ST.

10 Ơ ST (MNHN-EO-PHAS375) : [Indonésie], Bornéo, M. Chaper, $1891,124$.

1 ㅇ ST (MNHN-EO-PHAS374) : [Indonésie], Bornéo, M. Chaper, 1891,1287 [18]91, 116.

\section{REMARQUE}

Chaper a exploré la partie occidentale de Bornéo, autour du fleuve Kapocas (Kapuas), donc actuellement dans la partie indonésienne de l'île (Kalimatan).

\section{riveti Shelford, 1913 [Ocnophila]}

Ocnophila riveti Shelford, 1913: 62, pl. 3, fig. 6

STATUT ACTUEL. - Ocnophila riveti Shelford, 1913.

Matériel tYPe au MNHN. - HT 9.

HT क (MNHN-EO-PHAS626) : Équateur, El Pelado, $4150 \mathrm{~m}$. d'alt., P. Rivet, Janvier, 1903.

\section{rollandi Lucas, 1882 [Heteropteryx]}

Heteropteryx rollandi Lucas, 1882: 32.

STATUT ACTUEL. - Heteropteryx dilatata (Parkinson, 1798) - Kirby 1904b : 397 (synonymie).

Matériel TYPE AU MNHN. - HT o

HT $\%$ (MNHN-EO-PHAS349) : [Malaisie], Malacca, M. Rolland, 1912 Bis [18]81, 93.

rothschildi Bolívar, 1922 [Palophus]

Palophus rothschildi Bolívar, 1922: 210. 
STATUT ACTUEL. - Bactrododema phillipsi (Kirby, 1896) - Kirby 1896: 467 - Brock 2004: 65 (synonymie).

MATÉRIEL TYPE AU MNHN. - HT o .

HT (MNHN-EO-PHAS84): [Kenya], Afrique Orientale Angl[aise], Sud du Lac Rodolphe, Entre le chemin de fer et le lac, Maurice de Rothschild, 1905.

rotundatolobatus Brunner von Wattenwyl, 1907 [Carausius]

Carausius rotundatolobatus Brunner von Wattenwyl, 1907: 275.

StatUT ACTUEL. - Carausius rotundatolobatus Brunner von Wattenwyl, 1907.

MATÉRIEL TYPE AU MNHN. - 3 o ST.

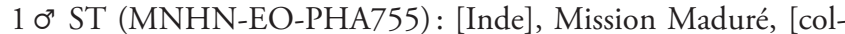
lection Pantel].

1 ơ ST (MNHN-EO-PHA756) : [Inde], Kodikanel [Kodaikanal], P. Décoly, 152, [collection Pantel].

1 ơ ST (MNHN-EO-PHA757) : [Inde], Indes, Kodikanel [Kodaikanal], P. Castets, [collection Pantel].

\section{REMARQUES}

Les 4 autres ơ de la collection Pantel sont probablement des individus d'élevage et non pas des ST. Ils portent respectivement les numéros suivant: 2 (MNHN-EO-PHA758), 4 (MNHN-EO-PHA759) (juvénile), 5 (MNHN-EOPHA760) et 6 (MNHN-EO-PHA761). D'autres ST au MNMS ; 1 ơ ST au NHMW (Brock 1998a; Brock \& Delfosse 2005).

\section{ruboligata Hennemann, Conle, Bellanger, Lelong \& Jourdan, 2018 [Phantasca]}

Phantasca ruboligata Hennemann, Conle, Bellanger, Lelong \& Jourdan, 2018: 50, figs 104-119.

Statut actuel. - Phantasca ruboligata Hennemann, Conle, Bellanger, Lelong \& Jourdan, 2018.

MATÉRIEL TYPE AU MNHN. - HT ơ, 2 ơ PT, 1 ㅇ PT.

HT o' (MNHN-EO-PHA1216): Guyane, Montagne de Kaw, Roubaud, Auvray, Rarchaert rec., 5-12.VIII.[19]92, [coll. GEP]. 1 ơ PT (MNHN-EO-PHA1217): Guyane, Montagne de Kaw, Roubaud, Auvray, Rarchaert rec., 5-12.VIII.[19]92, [coll. GEP]. $10^{7}$ PT (MNHN-EO-PHA1218): [Guyane], P. E. Roubaud det., 27.VII, A7 S1 N12, [coll. GEP].

1 ㅇ PT (MNHN-EO-PHA1219): [Guyane], [coll. GEP].

\section{REMARQUe}

Seuls quatre types sont actuellement au MNHN, 4 O' PT ( 2 dans la coll. YB et 2 dans la coll. PL) et 4 P PT (1 dans la coll. YB et 3 dans la coll. PL) viendront rejoindre la collection nationale du MNHN dans l'avenir. 24 ơ $^{7}$ et 1 P PT dans la collection OC et des œufs associés à des PT dans la collection FH (Hennemann et al. 2018). rudissimus Brunner von Wattenwyl, 1907 [Carausius]

Carausius rudissimus Brunner von Wattenwyl, 1907: 274.

Statut aCtUel. - Carausius rudissimus Brunner von Wattenwyl, 1907.

MatérIel TYPE AU MNHN. - 3 9 ST.

1 ST (MNHN-EO-PHA751): [Inde], Mission Maduré, 36, [collection Pantel].

1 ᄋ ST (MNHN-EO-PHA752): [Inde], Kodikanel [Kodaikanal], P. Décoly, [collection Pantel].

1 o ST (MNHN-EO-PHA753): [Inde], Ind[es] Or[ientales], P. Castets, [collection Pantel].

\section{ruficornis Redtenbacher, 1906 [Dina]}

Dina ruficornis Redtenbacher, 1906: 86, pl. 4, fig. 9.

STATUT aCtUEL. - Dinophasma ruficorne (Redtenbacher, 1906) - Brock 1998a: 54.

MATÉRIEL TYPE AU MNHN. - 1 ㅇ PLT.

1 P PLT (MNHN-EO-PHAS350): [Inde], Indes anglaises, Balasore, R. Oberthür, 1898, 7.

REMARQUES

Ainsi que le mentionne Bragg (2001), il est probable que ce spécimen soit mal étiqueté ou qu'il représente une autre espèce, le reste de la série étant de Bornéo. LT $ᄋ$, 1 \% PLT et $20^{7}$ PLT au NHMW; 2 ơ PLT et 4 \% PLT au ZIN (Brock 1998a, 2007; Brock et al. 2018). Le LT a été désigné par Bragg (2001: 250). Le nom de genre étant neutre, l'épithète spécifique doit être accordée en conséquence (accord rectifié).

rugulosus Redtenbacher, 1908 [Lamachus]

Lamachus rugulosus Redtenbacher, 1908: 483.

StatuT ACTUEL. - Orxines rugulosus (Redtenbacher, 1908) - Otte \& Brock 2005: 241

MATÉRIEL TYPE AU MNHN. - $10^{7}$ ST, $10^{\text {* ST. }}$

$10^{7}$ ST (MNHN-EO-PHAS798) : [Inde], Kurseong, Décoly, [18]98, [collection Pantel].

1 ơ ST (MNHN-EO-PHAS799): [Inde], Kurseong, Décoly, X.[18]99, [collection Pantel].

rulanda Redtenbacher, 1908 [Asceles]

Asceles rulanda Redtenbacher, 1908: 497.

STATUT ACTUEL. - Asceles rulanda rulanda Redtenbacher, 1908 Günther 1935a: 85; Otte \& Brock 2005: 54.

Matériel TYPe AU MNHN. - 1 \% ST.

1 ㅇ ST (MNHN-EO-PHAS1058) : N[ouv]elle Guinée, Dorey [Manokwari], Raffray et Maindron, 1878.

\section{REMARQUE}

1 Ơ $^{7}$ (MNHN-EO-PHAS382), Nouvelle-Calédonie, 1875, R. Germain, 1431, [18]75,130, possède une étiquette typique de l'auteur avec ce même nom mais la Nouvelle-Calédonie 
n'est pas répertoriée dans son ouvrage (Redtenbacher 1908). 3 ㅇ ST et 10 ơ ST au NHMW; 1 \& ST au ZMB; le matériel type du HNHM a été détruit dans un incendie (Brock 1998a).

sakavalanum Redtenbacher, 1906 [Parectatosoma]

Parectatosoma sakavalanum Redtenbacher, 1906: 165.

StatuT actuel. - Parectatosoma sakavalanum Redtenbacher, 1906.

MATÉRIEL TYPE AU MNHN. - 1 ơ ST, 1 9 ST.

$10^{7}$ ST (MNHN-EO-PHAS1059) et 1 \% ST et 4 œufs (MNHN-EO-

PHAS1060) : Madagascar, forêt de Sakavalana, Ch. Alluaud, 1901.

\section{REMARQUE}

1 ơ ST au NHMW (Brock 1998a; Cliquennois 2003a).

\section{scaberrimus Redtenbacher, 1906 [Pseudoleosthenes]}

Pseudoleosthenes scaberrimus Redtenbacher, 1906: 159, pl. 6, fig. 3. STATUT ACTUEL. - Pseudoleosthenes scaberrimus Redtenbacher, 1906.

MATÉRIEL TYPE AU MNHN. - 2 ㅇ PLT.

1 ㅇ PLT (MNHN-EO-PHAS89): Madag[ascar], F. Sikora, 1893, 906, 142.

1 ㅇ PLT (MNHN-EO-PHAS864) : Madagascar, collection Finot.

\section{REMARQUE}

Le LT $\%$ et la dernière 9 PLT sont au NHMW (Zompro 2002a; Cliquennois, 2003a). Le LT a été désigné par Zompro (2002a: 7).

scabra Redtenbacher, 1906 [Heteropteryx $]$

Heteropteryx scabra Redtenbacher, 1906: 171.

STATUT ACTUEL. - Haaniella scabra (Redtenbacher, 1906) - Rehn 1938: 369.

Matériel TYPE AU MNHN. - 1 \% ST, 1 \& ST juvénile.

1 \& ST (MNHN-EO-PHAS1165): [Malaisie], Nord Bornéo, KinaBalu, Collection A. Finot.

1 ㅇ ST (MNHN-EO-PHAS1166) (juvénile): [Malaisie], Nord Bornéo, Kina-Balu, 1500 m, H. Rolle, Collection A. Finot.

\section{REMARQUE}

Il s’agit de spécimens non cités par Redtenbacher (1906), ni de Paris, ni de la collection Finot, alors que l'étiquette de détermination est de sa main et que l'un des spécimens est de la collection Rolle, collection citée par le descripteur luimême. 1 ơ ST et 1 o ST au NHMW, 1 \& ST au ZIN (Brock 1998a, 2007; Brock et al. 2018).

$$
\text { sechellensis Bolívar, } 1895 \text { [Lonchodes] }
$$

STATUT ACTUEL. - Carausius sechellensis (Bolívar, 1895) - Brunner von Wattenwyl 1907: 274.

MATÉRIEL TYPE AU MNHN. - 4 O ST, 2 9 ST.

$20^{7} \& 2$ ㅇ ST (MNHN-EO-PHA1061, MNHN-EO-PHA1062, MNHN-EO-PHA1096, MNHN-EO-PHA1097): Seychelles, Mahé, Alluaud, 1892, 115-[18]96.

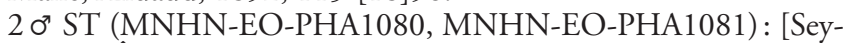
chelles], Îles Séchelles, Mahé, Ch. Alluaud, 1892, 115-[18]96.

\section{REMARQUES}

Dans le catalogue des entrées des spécimens au MNHN, Alluaud note, au numéro 115 de l'année 1896, 6 types de Lonchodes sechellensis conservés en alcool. C'est pourquoi nous avons retenu les 6 spécimens cités ci-dessus dont les numéros d'inventaire correspondent parfaitement et qui sont tous notés «type», même si deux d'entre eux ne sont pas en alcool, mais ont pu l'être par le passé. 1 \% récoltée en 1893 (MNHN-EO-PHA970) est présente dans la collection Pantel mais sans étiquette caractéristique de Bolívar ou de signe distinctif et le récolteur en est R. P. Capelle alors que d'après la publication Alluaud est le récolteur de tous les types. Une des 9 figurant parmi les ST est en fait Carausius alluaudi (Bolívar, 1895); cependant il paraît étonnant qu'il y ait eu une erreur étant donné que Bolívar fait bien la distinction entre $C$. alluaudi et $C$. sechellensis. 1 क ST et 1 Oे $^{\text {ST }}$ au MNMS (Brock 2007).

\section{sericeum Redtenbacher, 1906 [Abrosoma]}

Abrosoma sericeum Redtenbacher, 1906: 85.

STATUT ACtUEl. - Abrosoma sericeum Redtenbacher, 1906.

MATÉRIEL TYPE AU MNHN. - 1 9 ST.

1 ㅇ ST (MNHN-EO-PHAS1063): [Inde], Madras, J. Janssen, $87-[18] 72$.

REMARQUE

2 오 ST au NHMW (Brock 1998a).

\section{siamensis Brunner von Wattenwyl, 1907 [Clitumnus]}

Clitumnus siamensis Brunner von Wattenwyl, 1907: 193.

STATUT ACTUEL. - Ramulus siamensis (Brunner von Wattenwyl, 1907) - Brock 2003: 64

Matériel type AU MNHN. - $10^{7}$ ST, 2 \% ST.

10 O' ST (MNHN-EO-PHAS1076) : [Thaïlande], Siam, J. M. Bel, $1893,26,26=62$

1 ㅇ ST (MNHN-EO-PHAS1074): [Thaillande], Siam, J. M. Bel, 1893, 895 [18]93, 62.

1 ㅇ ST (MNHN-EO-PHAS1075): [Thaillande], Siam, J. M. Bel, 1893, 99.

$$
\text { siccaSjöstedt, } 1909 \text { [Gratidia] }
$$

Gratidia siccaSjöstedt, 1909: 81. 
STATUT ACtUel. - Clonaria sicca (Sjöstedt, 1909) - Otte \& Brock 2005: 105.

MATÉRIEL TYPE AU MNHN. - 4 9 ST.

1 \& ST (MNHN-EO-PHAS8): [Tanzanie], Kilimandjaro et Mérou, Kilimandjaro, Expédit[ion] Suédoise, Sjöstedt, Y. Sjöstedt, sept[embre], 1905-[190]6, 1912.

3 \& ST (MNHN-EO-PHAS9, MNHN-EO-PHAS10, MNHNEO-PHAS11): [Tanzanie], Kilimandjaro et Mérou, Kilimandjaro, Expédit[ion] Suédoise, Sjöstedt, Y. Sjöstedt, sept[embre], 1905 [190]6, 1912.

\section{REMARQUE}

10 \& ST au NHRS (K. A. Johanson comm. pers.), 1 \& ST au ZMBH (Brock et al. 2018). Il reste à localiser de nombreux ST car Sjöstedt (1909) cite $39 \%$ au total.

\section{significans Redtenbacher, 1908 [Bacteria]}

Bacteria significans Redtenbacher, 1908: 422.

STATUT ACTUEL. - Phanocles significans (Redtenbacher, 1908) Conle et al. 2011: 60 .

MATÉRIEL TYPE AU MNHN. - HT 9.

HT + (MNHN-EO-PHAS576) : [Colombie], Bogota, 201-[18]62, $201[18] 62,156$.

\section{simile Redtenbacher, 1908 [Xenophasma]}

Xenophasma simile Redtenbacher, 1908: 442.

STATUT ACTUEL. - Xenophasmina similis (Redtenbacher, 1908) Brock 1998a: 58 [Xenophasmina simile (Redtenbacher, 1908) (sic)].

Matériel type aU MNHN. - 1 \% ST.

1 o ST (MNHN-EO-PHAS423) : [Vietnam], Tonkin, Bac-Kan, Ct. Roger, 1897, 64.

\section{REMARQUE}

1 \& ST au NHMW (Brock 1998a). Uvarov a créé ce nom de genre en substituant à la finale grecque le suffixe latin -ina. Ce suffixe étant féminin, le nom de genre l'est aussi et l'épithète spécifique doit être accordé en conséquence.

simplex Redtenbacher, 1906 [Antongilia]

Antongilia simplex Redtenbacher, 1906: 26.

STATUT ACTUEL. — Virgasia simplex (Redtenbacher, 1906) - Cliquennois 2006: 164.

MATÉRIEL TYPE AU MNHN. -8 ơ PLT, 5 9 PLT.

$80^{7}$ PLT dont 2 juvéniles (MNHN-EO-PHAS917, MNHN-EOPHAS 921, MNHN-EO-PHAS924, MNHN-EO-PHAS925, MNHN-EO-PHAS926, MNHN-EO-PHAS927, MNHN-EOPHAS922, MNHN-EO-PHAS923), 5 \% PLT dont 2 juvéniles (MNHN-EO-PHAS918, MNHN-EO-PHAS928, MNHN-EOPHAS 929, MNHN-EO-PHAS919, MNHN-EO-PHAS920) : Madagascar, Maroancetra [Maroantsetra], 12.1897, collection Finot.
REMARQUE

LT o", 3 o PLT et 4 ㅇ PLT au NHMW; $30^{*}$ PLT et 19 PLT (juvénile) au ZMH; $20^{\prime \prime}$ PLT et 2 PLT (dont 1 juvénile) au ZIN (Zompro 2002b; Cliquennois 2003a, 2006; Brock 2007). Le LT a été désigné par Cliquennois (2003a: 7).

\section{simplicipes Audinet-Serville, 1838 [Pachymorpha]}

Pachymorpha simplicipes Audinet-Serville, 1838: 259.

StATUT ACtUEL. - Pachymorpha simplicipes Audinet-Serville, 1838.

Matériel TYPe AU MNHN. - $10^{\pi}$ ST.

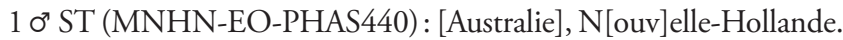

REMARQUE

Audinet-Serville (1838) note aussi 1 \% ST qui n’a pas été retrouvée.

sinetyi Brunner von Wattenwyl, 1907 [Phobaeticus]

Phobaeticus sinetyi Brunner von Wattenwyl, 1907: 184.

STATUT ACtUel. — Phobaeticus sinetyi Brunner von Wattenwyl, 1907.

MatéRIEL tYPE AU MNHN. - 1 ơ PLT, 1 9 PLT.

$10^{7}$ PLT (MNHN-EO-PHAS731), 1 PLT (MNHN-EOPHAS732): [Inde], Kodikanel [Kodaikanal], P. Décoly, [collection Pantel].

\section{REMARQUES}

Une autre $\$$ (MNHN-EO-PHAS733), collection Pantel, présente uniquement une étiquette " $\odot$ authentique». $7 \sigma^{*}$ (MNHN-EO-PHAS971, MNHN-EO-PHAS972, MNHNEO-PHAS973, MNHN-EO-PHAS974, MNHN-EOPHAS975, MNHN-EO-PHAS976, MNHN-EO-PHAS977), et 3 (MNHN-EO-PHAS978, MNHN-EO-PHAS979 et leurs œufs MNHN-EO-PHAS981, MNHN-EO-PHAS980), également présents dans la collection Pantel sont des individus d'élevage. LT ơ', 1 \% PLT et un œuf au NHMW (Brock 1998a; Brock \& Delfosse 2005). Le LT a été désigné par Hennemann \& Conle (2008: 188).

singulare Redtenbacher, 1906 [Abrosoma]

Abrosoma singulare Redtenbacher, 1906: 85.

STATUT ACTUEL. - Abrosoma singulare Redtenbacher, 1906.

MATÉRIEL TYPE AU MNHN. - 1 ơ ST, 2 \% ST.

1 ơ ST (MNHN-EO-PHAS712): [Inde], Kodikanel [Kodaikanal, P. Décoly], 27, [collection Pantel].

1 ㅇ ST (MNHN-EO-PHAS713) : [Inde], Décoly, [18]97, 27, [collection Pantel].

1 ㅇ (MNHN-EO-PHAS716) : [cette $\$$ et le ơ MNHN-EOPHAS712] accouplés, $n^{\circ}$ 27, [collection Pantel]. 
REMARQUE

2 (MNHN-EO-PHAS714, MNHN-EO-PHAS715) de la collection Pantel sont sans étiquette et sont de statut incertain. $10^{7}$ ST au MNMS; 1 \% ST et $10^{7}$ ST au NHMW (Brock 1998a; Brock \& Delfosse 2005).

\section{solida Redtenbacher, 1908 [Sosibia]}

Sosibia solida Redtenbacher, 1908: 536.

STATUT ACTUEL. - Planososibia lysippus (Westwood, 1859) - SeowChoen 2016: 224 (synonymie).

MATÉRIEL TYPE AU MNHN. - HT + .

HT + (MNHN-EO-PHAS369): [Malaisie], Malacca, Erington de L. $1899,183$.

sollicita Redtenbacher, 1908 [Necroscia]

Necroscia sollicita Redtenbacher, 1908: 563.

STATUT ACTUEl. - Calvisia conicipennis (Bates, 1865) - SeowChoen 2016: 51 (synonymie).

Matériel TyPe au MNHN. - HT ơ.

HT ơ (MNHN-EO-PHAS376) : [Indonésie], Bornéo, M. Chaper, 1891, 1285 [18]91, 93.

\section{REMARQUE}

Chaper a exploré la partie occidentale de Bornéo, autour du fleuve Kapocas (Kapuas), donc actuellement dans la partie indonésienne de l'île (Kalimatan). La localité précise est "Vallée du fleuve Kapuas», d’après Chaper (1891).

\section{spiniger Brunner von Wattenwyl, 1907 [Clitumnus]}

Clitumnus spiniger Brunner von Wattenwyl, 1907: 191.

STATUT ACTUEL. - Parapachymorpha spinigera (Brunner von Wattenwyl, 1907) - Brock 2003: 54.

MatéRIEL TYPe AU MNHN. - HT ơ.

HT ơ (MNHN-EO-PHAS389) : [Vietnam], Tonkin, R. Oberthür, 1902.

spinosa Cliquennois \& Brock, 2004 [Monoiognosis]

Monoiognosis spinosa Cliquennois \& Brock, 2004b : 8, figs 17-24, 35.

STATUT ACTUEL. - Monoiognosis spinosa Cliquennois \& Brock, $2004 \mathrm{~b}$.

MATÉRIEL TYPE AU MNHN. - HT ơ, 1 \& PT.

HT ơ (MNHN-EO-PHAS1204): Mauritius [Maurice], [Plateau de] Mare Longue (parcelle grillagée), On Leea guineensis, [alt. 500 m], 15.iii.2001, NCMR33, N. Cliquennois, NCMR33.

1 \& PT (MNHN-EO-PHAS1205): Mauritius [Maurice], [Plateau de] Mare Longue, On Leea guineensis, [alt. 500 m], 11.III.2001, N. Cliquennois, NCMR20.

\section{REMARQUE}

$10^{x}$ PT et 1 P PT au NHMUK; $10^{x}$ PT et 1 ㅇ PT au MHNR.

\section{spinulosa Chopard, 1952 [Parectatosoma]}

Parectatosoma spinulosa Chopard, 1952: 480.

Statut actuel. - Paranisacantha spinulosa (Chopard, 1952) Cliquennois 2008: 66.

Matériel TYPE AU MNHN. - HT $\%$.

HT \& (MNHN-EO-PHAS180) : Madagascar, M[on]t Tsaratanana, forêt de mousses, $1500 \mathrm{~m}, \mathrm{X}-[19] 49$, R. P[aulian], Inst[itut]. Scient[ifique]. [de] Madagascar.

spinulosus Redtenbacher, 1908 [Oxyartes]

Oxyartes spinulosus Redtenbacher, 1908: 475.

STATUT ACtUEL. - Oxyartes spinulosus Redtenbacher, 1908 (nouveau statut).

MatéRIel TYPE AU MNHN. - HT o".

HT ơ (MNHN-EO-PHAS397): [Vietnam], Tonkin Centr[e], Env[irons] de Tuyen-Quan, A. Weiss, printemps, 1901.

\section{REMARQUE}

Oxyartes spinulosus est une espèce valide, bien que Dohrn (1910) l'ait mise en synonymie avec Oxyartes lamellatus Kirby, 1904. L'examen du spécimen type montre qu'il s'agit d'une espèce distincte, plus grande et plus fine que tous les individus $0^{\prime}$ d'O. lamellatus observés. O. spinulosus s'en distingue en outre par l'absence d'ailes.

\section{spurcata Brunner von Wattenwyl, 1907 [Dyme]}

Dyme spurcata Brunner von Wattenwyl, 1907: 326.

StatuT aCtUel. - Candovia spurcata (Brunner von Wattenwyl, 1907) - Brock \& Hasenpusch 2007: 8.

MatéRIEL TYPE AU MNHN. - HT ơ.

HT ơ (MNHN-EO-PHAS426) : [Australie], N[ouve]lle Hollande.

\section{squamigera Redtenbacher, 1906 [Antongilia]}

Antongilia squamigera Redtenbacher, 1906: 27, pl. 2, figs 12, 13.

STATUT ACTUEL. - Paronogastris squamigera (Redtenbacher, 1906) - Cliquennois 2006: 162.

MATÉRIEL TYPE AU MNHN. - 1 Ơ PLT.

10 ơ PLT (MNHN-EO-PHAS102): Madagascar, Tamatave, A. Mathiaux, 1898, 103.

REMARQUE

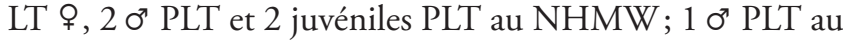
ZIN (Brock 1998a; Cliquennois 2003a, 2006; Brock et al. 2018). Le LT a été désigné par Cliquennois (2003a: 8).

strumosa Brunner von Wattenwyl, 1907

[Paradiapheromera]

Paradiapheromera strumosa Brunner von Wattenwyl, 1907: 317. 
STATUT ACTUEL. - Oncotophasma martini (Griffini, 1896) : 10 Hebard 1923: 358 (synonymie).

MATÉRIEL TYPE AU MNHN. - 10 $0^{\text {P PLT. }}$

$10^{7}$ PLT (MNHN-EO-PHAS599): [Panama], Darien [Darién], F. Geay, 1899.

\section{REMARQUE}

LT ơ et 1 ơ PLT au NHMW (Brock 1998a; Zompro 2007).
Matériel TYPE AU MNHN. - 1 \% PLT.

1 ㅇ PLT (MNHN-EO-PHAS351) : Bornéo, R. Oberthür, 1898, 11.

REMARQUE

La mention «Bornéo" ne permet pas pour ce spécimen de déterminer s'il provient de Malaisie, d'Indonésie ou de Brunei. LT ơ, $20^{7}$ PLT et 2 PLT au NHMW (Brock 1998a; Brock et al. 2018).

tecticollis Redtenbacher, 1908 [Vasilissa]

Vasilissa tecticollis Redtenbacher, 1908: 383, pl. 17, fig. 6.

STATUT ACTUEL. - Tropidoderus rhodomus MacCoy, 1882 - Hennemann et al. 2015: 38 (synonymie).

MATÉRIEL TYPE AU MNHN - 1 O ST.

1 \& ST (MNHN-EO-PHAS641) : [Sri Lanka], Ceylon?, P. Michel, 1888, 33 [collection Pantel].

\section{REMARQUE}

La seconde 9 ST est au MNMS (Brock \& Delfosse 2005).

\section{substrumosus Redtenbacher, 1908 [Hypocyrtus]}

Hypocyrtus substrumosus Redtenbacher, 1908: 357.

STATUT ACTUEL. - Hypocyrtus postpositus (Redtenbacher, 1908) 356 - Hennemann \& Conle 2012: 72 (synonymie).

MATÉRIEL TYPE AU MNHN. - 3 \% PLT.

1 ㅇ PLT (MNHN-EO-PHAS520) : Mexique, A. Sallé, 60-[18]56, 145 , gardé 1 ex.

1 ㅇ PLT (MNHN-EO-PHAS521) : Mexique, A. Sallé, 60-[18]56. 1 o PLT (MNHN-EO-PHAS522) (juvénile) : Mexique, A. Sallé, $60-[18] 56,60[18] 56$.

\section{REMARQUE}

LT $q$ et 2 PLT $q$ (dont 1 juvénile) au NHMW; 1 PLT au NHMUK; 1 o PLT au ZMH (Brock 1998a; Zompro 2002b). Hennemann \& Conle (2012) note un autre PLT o (juvénile) au NHMUK. Le LT a été désigné par Hennemann \& Conle (2012: 72).

superba Redtenbacher, 1908 [Loxopsis]

Loxopsis superba Redtenbacher, 1908: 503.

STATUT ACtUEL. — Loxopsis superba Redtenbacher, 1908.

MATÉRIEL TYPE AU MNHN. - HT ㅇ.

HT 9 (MNHN-EO-PHAS384): [Indonésie], Bornéo, Riv[ière] Mandore, R. Oberthür, 1898, 76.

superbum Redtenbacher, 1906 [Ascepasma]

Ascepasma superbum Redtenbacher, 1906: 77.

STATUT ACTUEL. - Orthomeria (Orthomeria) superba (Redtenbacher, 1906) - Günther 1935b: 4.
MatéRIEL TYPE AU MNHN. - HT ơ.

HT ơ (MNHN-EO-PHAS404) : Philippines, 136.

\section{REMARQUE}

Le genre Tropidoderus étant endémique d'Australie, la localité «Philippines» est probablement erronée (Hennemann et al. 2015).

terminalis Redtenbacher, 1908 [Necroscia]

Necroscia terminalis Redtenbacher, 1908: 561, pl. 27, fig. 6.

STATUT ACTUEL. - Paranecroscia terminalis (Redtenbacher, 1908).

MATÉRIEL TYPE AU MNHN. $-10^{7}$ ST.

1 o' ST (MNHN-EO-PHAS636) : [Malaisie], Nord Bornéo, O. Staudinger, 1898, [collection Pantel].

REMARQUE

1 ㅇ (MNHN-EO-PHAS637) de la collection Pantel, sans étiquette, est de statut incertain. 6 o ST et $40^{7}$ ST au NHMW (Brock 1998a).

texanus Brunner von Wattenwyl, 1907 [Bacunculus]

Bacunculus texanus Brunner von Wattenwyl, 1907: 333.

STATUT ACTUEL. - Diapheromera (Diapheromera) persimilis Caudell, 1904: 107 - Hebard 1943: 299 (synonymie).

MATÉRIEL TYPE AU MNHN. -1 \& ST.

1 \& ST (MNHN-EO-PHAS596): [États-Unis], New York, N.Y., A. Sallé, 1886, 4638 [18]86, 78.

REMARQUE

1 ㅇ ST et 1 ơ ST au NHMW ; 1 ㅇ ST au ZMB (Brock 1998a). La \& ST de New York est soit mal étiquetée, soit une espèce distincte d'après Hebard (1943). - Seow-Choen 2016: 215. 
tobagoensis Langlois \& Bellanger, 2012 [Bostra]

Bostra tobagoensis Langlois \& Bellanger, 2012: 98, figs 7, 8, 31-36, 49-51.

STATUT ACTUEL. — Bostra tobagoensis Langlois \& Bellanger, 2012

MATÉRIEL TYPE AU MNHN. - HT $\%$, AT $0^{n}$.

HT $\%$ (MNHN-EO-PHAS588) : [Trinité et Tobago], Tobago, trace à $3,2 \mathrm{~km}$ à l'ouest de Castara, route de Plymouth, F. Langlois \& Y. Bellanger, 23.X.2008, TOB08-010.

AT ơ (MNHN-EO-PHAS589): [Trinité et Tobago], Tobago, élevage ex-ovo issu de l'holotype et de paratypes $\$$, F. Langlois \& Y. Bellanger, 01.XI.2009, TOB08-110.

\section{REMARQUE}

2 ơ PT et 2 우 PT dans la collection CFL (Langlois \& Bellanger 2012).

\section{triangulifera Redtenbacher, 1906 [Oestrophora]}

Oestrophora triangulifera Redtenbacher, 1906: 124, pl. 5, fig. 2.

StatuT aCtUel. — Oestrophora triangulifera Redtenbacher, 1906.

MATÉRIEL TYPE AU MNHN. - 1 ST $0^{*}$.

1 ST o" (MNHN-EO-PHAS517): Guyane, Cayenne, Banon, 9653-[18]34, 86.

\section{REMARQUE}

$10^{7} \mathrm{ST}$ au MHNG; $10^{7}$ ST et 1 ㅇ ST au NHMW (Brock 1998a; Zompro \& Brock 2003).

\section{trilineatus Brunner von Wattenwyl, 1907 [Clitumnus]}

Clitumnus trilineatus Brunner von Wattenwyl, 1907: 191, pl. 8, fig. 2a, b.

STATUT ACTUEL. - Ramulus trilineatus (Brunner von Wattenwyl, 1907) - Otte \& Brock 2005: 307.

Matériel TYPe AU MNHN. -20 ST.

$20^{7}$ ST (MNHN-EO-PHAS1064, MNHN-EO-PHAS1065) : [Sri

lanka], Ceylan, E. Deschamps, 1898-1889.

\section{REMARQUE}

Brunner von Wattenwyl (1907) ne fait pas mention du Muséum de Paris, pourtant il s'agit bien de spécimens déterminés par lui-même comme le montre l'étiquette originale.

\section{tuberculata Redtenbacher, 1908 [Loxopsis]}

Loxopsis tuberculata Redtenbacher, 1908: 503.

StatuT ACtUel. - Paraloxopsis tuberculata (Redtenbacher, 1908) - Bragg 2006: 6.

MATÉRIEL TYPE AU MNHN. - 1 \% ST.

1 9 ST (MNHN-EO-PHAS383): [Indonésie], Bornéo sept[entrional], Pontianak, R. Oberthür, 1897.
REMARQUE

Le ơ ST n'a pas été retrouvé au MNHN.

tuberculatum Brunner von Wattenwyl, 1907 [Prisomera]

Prisomera tuberculatum Brunner von Wattenwyl, 1907: 287.

StatUT ACtUEL. - Mnesilochus mindanaensis (Brunner von Wattenwyl, 1907) - Hennemann \& Conle 2007: 70 (synonymie) [Mnesilochus mindanaense (Brunner von Wattenwyl, 1907) (sic)].

MATÉRIEL TYPE AU MNHN. - HT 9 .

HT (MNHN-EO-PHAS1066) : [Philippines], Mindanao, J. Montano, 158-[18]81.

REMARQUE

Le nom de genre Mnesilochus étant masculin, l'épithète spécifique doit être accordée en conséquence.

\section{vacillans Brunner von Wattenwyl, 1907 [Carausius]}

Carausius vacillans Brunner von Wattenwyl, 1907: 271.

StatuT ACtUel. - Carausius vacillans Brunner von Wattenwyl, 1907.

MATÉRIEL TYPE AU MNHN. - 3 9 ST.

1 ㅇ ST (MNHN-EO-PHAS743): [Inde], Ind[es] or[ientales], P. Castets, [collection Pantel].

1 \& ST (MNHN-EO-PHAS744): [Inde], Indes, Kodikanel [Kodaikanal], P. Castets, [collection Pantel].

1 ST (MNHN-EO-PHAS745): [Inde], Shembaganur, J. Dubreuil, [collection Pantel].

REMARQUE

1 ㅇ ST au MNMS : Madura (Brock \& Delfosse 2005).

\section{vaginata Redtenbacher, 1906 [Orobia]}

Orobia vaginata Redtenbacher, 1906: 70.

STATUT ACTUEL. - Leiophasma vaginatum (Redtenbacher, 1906) - Brock 1998a: 64.

MATÉRIEL TYPE AU MNHN. - 7 ㅇ ST.

1 \& ST (MNHN-EO-PHAS252): Madagascar, Saint-Marie de Madagascar, collection Finot.

5 9 ST (MNHN-EO-PHAS258, MNHN-EO-PHAS884, MNHNEO-PHAS885, MNHN-EO-PHAS886, MNHN-EO-PHAS887) : Madagascar, Maroancetra [Maroantsetra], 12.1897, collection Finot. 1 ST (MNHN-EO-PHAS1067) : Madagascar, Lantz, 241-[18]82.

REMARQUE

2 ㅇ et $10^{7}$ ST au NHMW ; 1 et $10^{7} \mathrm{ST}$ au ZMH; $10^{\prime \prime} \mathrm{ST}$ à l'ANSP; 1 ᄋ ST au ZIN (Zompro, 2002b; Cliquennois, 2003a; Brock, 2007).

verruculatus Redtenbacher, 1906 [Orestes]

Orestes verruculatus Redtenbacher, 1906: 47, pl. 1, fig. 12. 
STATUT ACtUel. - Orestes mouhotii (Bates, 1865) - Bates 1865: 342 - Zompro 2004: 222 (synonymie).

MATÉRIEL TYPE AU MNHN. - HT 9.

HT \& (MNHN-EO-PHAS340) (juvénile) : [Thaïlande], Bangkok, J. Harmand, 1885, 7990 [18]85, 47, 47.

vignieri Redtenbacher, 1906 [Xerosoma]

Xerosoma vignieri Redtenbacher, 1906: 144.

STATUT ACTUEL. — Isagoras vignieri (Redtenbacher, 1906)] - Hebard 1923: 351.

Matériel TYPe AU MNHN. - HT ơ.

HT ơ (MNHN-EO-PHAS1068) : [Panama], Ie du Darien, Paya, Vignier, 874-[18]78.

\section{villosa Redtenbacher, 1908 [Parasosibia]}

Parasosibia villosa Redtenbacher, 1908: 482.

Statut aCtuel. - Parasosibia villosa Redtenbacher, 1908.

MATÉRIEL TYPE AU MNHN. - 11 O ST.

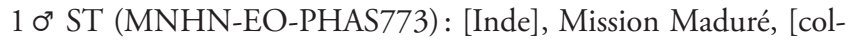
lection Pantel].

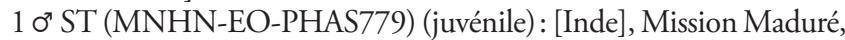
Shembaganor [Shembaganur], VIII.[19]00, 8, [collection Pantel]. 3 o' ST dont 1 juvénile (MNHN-EO-PHAS789, MNHN-EOPHAS781, MNHN-EO-PHAS780) (juvénile): [Inde], Mission Maduré, [collection Pantel].

3 ơ ST (MNHN-EO-PHAS790, MNHN-EO-PHAS791, MNHNEO-PHAS792): [Inde], Ind[es] or[ientales], P. Castets, [collection Pantel].

1 ơ ST (MNHN-EO-PHAS793): [Inde], Ind[es] or[rientales], R. P. Capelle, 1889, [collection Pantel].

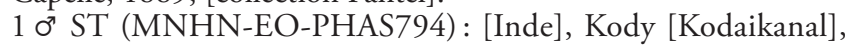
Décoly [18]93, [collection Pantel].

1 ơ ST (MNHN-EO-PHAS795) : [Inde], Kodikanel [Kodaikanal], Décoly, [collection Pantel].

\section{REMARQUES}

Dans la collection Pantel, 10 autres ơ (MNHN-EO-PHAS774, MNHN-EO-PHAS775, MNHN-EO-PHAS776, MNHNEO-PHAS782, MNHN-EO-PHAS783, MNHN-EOPHAS784, MNHN-EO-PHAS785, MNHN-EO-PHAS786, MNHN-EO-PHAS787, MNHN-EO-PHAS788) et 1 ㅇ (MNHN-EO-PHAS778) sans étiquette; 1 \% (MNHN-EOPHAS777), notée "type», possède les mêmes données que le ST ơ, et a bien été examinée par Redtenbacher (1908), comme le confirme une étiquette rédigée de sa main, mais ne figure pas dans la description de l'espèce et ne peut être considérée comme un type. D’autres ST au MNMS dans la collection Bolívar (Brock \& Delfosse 2005).

\section{villosipes Redtenbacher, 1906 [Dinelytron]}

Dinelytron villosipes Redtenbacher, 1906: 151.

Statut actuel. - Dinelytron villosipes Redtenbacher, 1908.
Matériel tYPe au MNHN. - HT ơ.

HT ơ (MNHN-EO-PHAS1069): Brésil, Minas Gerais, Caraca,

E. Gounelle, 9112-[18]89.

\section{villosum Redtenbacher, 1906 [Abrosoma]}

Abrosoma villosum Redtenbacher, 1906: 85.

STATUT ACTUEL. - Coloratobistus villosus (Redtenbacher, 1906) Zompro 2004: 82.

MatéRIEL TYPE AU MNHN. - LT $\$$, 1 PLT.

LT \& (MNHN-EO-PHAS710): [Inde], Kodikanel [Kodaikanal], P. Décoly, [collection Pantel].

1 PLT (MNHN-EO-PHAS711): [Inde], Ind[es] or[ientales], P. Castets, [collection Pantel].

REMARQUE

$10^{7}$ PLT au NHMW (Brock \& Delfosse 2005). Le LT a été désigné par Brock (1998a: 65). Le nom de genre étant masculin, l'épithète spécifique doit être accordée en conséquence (accord rectifié).

\section{virga Redtenbacher, 1908 [Hermarchus]}

Hermarchus virga Redtenbacher, 1908: 446.

STATUT ACTUEL. - Hermarchus virga Redtenbacher, 1908.

MATÉRIEL TYPE AU MNHN. - 1 \% ST.

1 ㅇ ST (MNHN-EO-PHAS1093): I[le] Fidji, H. Filhol, 530-[18]76.

REMARQUES

1 ST $\%$ au NHMW (Brock et al. 2018).

viridipes Redtenbacher, 1906 [Prexaspes]

Prexaspes viridipes Redtenbacher, 1906: 129.

Statut aCtUel. - Prexaspes (Elasia) viridipes Redtenbacher, 1906 - Zompro 2004: 102.

MATÉRIEL TYPE AU MNHN. - HT $\%$

HT 9 (MNHN-EO-PHAS504) : Pérou, Yurimaguas, Ponce, A. Sallé $1886,4648[18] 86,53$.

\section{DISCUSSION}

Décider qu'un spécimen a le statut de type ou non est parfois difficile. Les auteurs anciens fournissaient rarement d'information sur le nombre d'individus étudiés ou considérés comme des types lorsqu'ils décrivaient une espèce. Ainsi, le plus souvent Brunner von Wattenwyl et Redtenbacher ne plaçaient une étiquette "type» que sous un seul des spécimens d'une espèce donnée pour signaler la série entière. Cependant, il leur arrivait aussi de disposer une étiquette sur plusieurs individus de la même espèce sans raison particulière. Savoir 
si ces auteurs ont bien étudié tous les spécimens d'une série donnée n'est donc pas aisé.

De même, les auteurs ont souvent fait don d'une partie de leurs spécimens une fois un taxon publié, sans pour autant signaler ces dons dans une publication. Ainsi, Brunner von Wattenwyl et Redtenbacher ont-ils pu donner des spécimens à Finot postérieurement à la publication de leur monographie. Cette pratique courante explique notamment que le MNHN possède des types de Sjöstedt. Partager les spécimens types est une pratique qui perdure encore aujourd'hui sous la forme de dépôt de types dans différentes institutions lors de la description, notamment lors de collaborations internationales.

Nous avons tenté de résoudre au mieux ces difficultés, d'une part en réexaminant chacune des descriptions originales en regard des spécimens de la collection et de leurs étiquettes, et d'autre part en justifiant nos hypothèses et nos doutes dans le champ «remarques» lorsque cela s'est avéré nécessaire.

Lors de l'étude, nous avons pu constater que les collections de phasmes d'Olivier sont absentes alors que les Coléoptères de la collection de cet auteur ont été rapatriés au MNHN. Les collections de phasmes d'Audinet-Serville sont également peu présentes car elles ont été vendues. Enfin, les phasmes décrits par Montrouzier, dont le fameux Dryococelus australis (Montrouzier, 1855), n’ont jamais été au MNHN. Les collectes de Montrouzier ont eu à subir divers aléas et sont perdues pour une bonne part (Schouteden 1907; Damoiseau 1966). Les spécimens sur lesquels cet auteur a fondé sa description de $D$. australis appartenaient à un Australien (Montrouzier 1855) et n'ont peut-être jamais quitté l'Australie. L'hypothèse selon laquelle, ils étaient dans la collection Mulsant à Saint-Chamond et y auraient été détruits (Vickery 1983) n'apparaît pas fondée.

La collection de phasmes du MNHN s'enrichit continuellement avec de nouveaux spécimens, par le biais de missions ou de dons, mais aussi avec de nouveaux types décrits par des spécialistes. Avec le développement de l'ootaxinomie, la collection d'œufs de phasmes du MNHN a également été grandement enrichie ces dernières années puisqu'elle est passée d'une dizaine d'espèces représentées à plus de 350 . Le présent catalogue vise à faciliter le travail des chercheurs en mettant les ressources de la collection à disposition de la communauté, à travers notamment la liste des types présent, leur informatisation et leur digitalisation.

\section{Remerciements}

Nous tenons tout d'abord à rendre hommage à la regrettée Christiane Amédégnato (CNRS), ainsi qu'à Simon Poulain (CNRS), qui ont grandement contribué à initier ce travail, notamment en donnant accès à la collection de phasmes du MNHN au premier auteur, et l'ont toujours encouragé à poursuivre ses travaux tout en le conseillant et en l'assistant techniquement. Nous remercions Paul Brock pour ses commentaires constructifs, ainsi qu'un reviewer anonyme. Nous sommes également les débiteurs de Kjell Arne Johanson (NHRS) pour les compléments d'informations apportés concernant les collections de Sjöstedt, mais aussi de Jocelyne
Guglielmi et Jacques Couverchel (bibliothèque d'Entomologie, MNHN) pour l'aide précieuse qu'ils nous ont apportée dans le domaine de la bibliographie et Joachim Bresseel pour ses précieuses informations taxinomiques. Ce projet a été réalisé dans le cadre du projet E-ReColNat (ANR-11-INBS-0004).

\section{RÉFÉRENCES}

Audinet-Serville J. G. 1831. - Revue méthodique de l'ordre des Orthoptères. Annales des Sciences Naturelles tome 22e, Paris Crochard, Librairie Editeur: 28-29, 56-65. https://biodiversitylibrary. org/page/6095939

Audinet-Serville J. G. 1838 [1839]. - Histoire naturelle des insectes orthoptères. Librairie encyclopédique de Roret: 9, 214-293, planches 6-8. https://doi.org/10.5962/bhl.title.16081

BAtEs H. W. 1865. - Description of 52 new species of Phasmidae from the collection of Mr W. Wilson Saunders with remarks on the family. Transactions of the Linnean Society of London 25 (1): 321-359.

BeIER M. 1957. - Cheleutoptera, in Bronns (H. G.), Klassen und ordnungen das Tierreichs 5: Arthropoda/III: Insecta 6: Orthopteroidea 2, Leipzig: 305-457. https://doi.org/10.5962/bhl.title.14134

BeIER M. 1968. - Phasmida (Stab-oder Gespenstheuschrecken), Handbuch der zoologie 4 (2) - Arthropoda Insecta. Walter \& Gruyter: 1-56.

Bellanger Y. 2016. - A new stick insect of the genus Oncotophasma from Costa Rica (Phasmatodea, Diapheromeridae, Diapheromerinae). Bulletin de la Société entomologique de France 121 (2): 141-148.

Bellanger Y. Jourdan T. \& Lelong P. 2012. - Contribution à l'inventaire et à la biologie des Phasmatodea de Trinidad. Bulletin de la Société entomologique de France 117 (4): 483-502.

BERG C. 1899. - Sobre algunos Anisomórfidos chileno-argentinos. Communicaciones del Museo Nacional de Buenos Aires 1 (5): 181-186.

BLANCHARD E. 1851a. - Fauna chilena - Insectos. Orden IV. Ortopteros, in GAY C., Historia fisica y politica de Chili, Zool. 6, Paris: 21-29. https://doi.org/10.5962/bhl.title.16172

Blanchard E. 1851b. - In GaY C., Historia de Chile - Orthoptères et Névroptères - Atlas. Imprimerie Lesauvage: planche 1. https:// doi.org/10.5962/bhl.title.16172

BLANCHARD E. 1853. - Insectes, in HOMBRON \& JACQUINOT (Charles Hector), Voyage au Pôle sud et dans l'Océanie sur les Corvettes l'Atrolabe et la Zélée; exécuté par ordre du Roi pendant les années 1837-1838-1839-1840, sous le commandement de M. J. Dumont-D'Urville, capitaine de vaisseau, publiépar ordre du gouvernement, sous la direction supérieure de M. Jacquinot, capitaine de vaisseau, commandant de la Zélée, Zoologie tome 4, P. Duméril éditeur: 358-360, planche 1, figures $8 \& 9$.

Boisduval A. D. DE 1835. - Faune entomologique de l'Océanie comprenant les Coléoptères, les Hémiptères, les Névroptères, les Hyménoptères et les Diptères. Librairie encyclopédique de Roret: 647-649, planche 10: 2. https://doi.org/10.5962/bhl.title.65987

BOLIVAR I. 1895. - Mission scientifique de M. Ch. Alluaud aux Iles Séchelles (mars-avril-mai 1892). Annales de la Société Entomologique de France 64: 372-375.

BolIVAR I. 1919. - Diagnoses d'Orthoptères nouveaux (Phasmidae, Locustidae) de l'Afrique tropicale. Bulletin de la Société entomologique de France: 241-243.

BOLIVAR I. 1922. — Voyage de M. Le baron Maurice de Rothschild en Ethiopie et en Afrique Orientale Anglaise (1904-1905) - Résultats scientifiques - Animaux articulés. Paris, Imprimerie Nationale: 205-211, planche 10. https://doi.org/10.5962/bhl.title.66850

BradLer S. 2009. — Die Phylogenie der Stab- und Gespenstschrecken (Insecta: Phasmatodea). Spezies, Phylogenie und Evolution 2.1: $139 \mathrm{p}$. 
BRAGG P. E. 1991. - Spermatophores in Phasmida. Entomologist 110 (2): 76-80.

BragG P. E. 1993. - New synonyms and new records of phasmids (Insecta: Phasmida) in Borneo. Raffles Bulletin of Zoology 41 (1): 31-46.

BRAGG P. E. 1998. - A revision of the Heteropteryginae (Insecta: Phasmida: Bacillidae) of Borneo, with the description of a new genus and ten new species. Nationaal Natuurhistorisch Museum: 3-135.

Bragg P. E. 2001. — Phasmids of Borneo. Natural History Publications (Borneo), Kota Kinabalu: 772 p.

Bragg P. E. 2006. - A review of Paraloxopsis Gunther, 1932 and a first description of the male and egg of Paraloxopsis korystes Gunther, 1932. Phasmid Studies 14 (1-2): 1-11.

BresseEl J. \& Constant J. 2014. - Giant Sticks from Vietnam and China, with three new taxa including the second longest insect known to date (Phasmatodea, Phasmatidae, Clitumninae, Pharnaciini). European Journal of Taxonomy 104: 1-38. https:// doi.org/10.5852/ejt.2014.104

BRESSEEL J. \& CONSTANT J. 2018. — Two new stick insect genera from Vietnam, Nuichua gen. nov. and Pterohirasea gen. nov. with two new species (Phasmida: Diapheromeridae: Necrosciinae). Belgian Journal of Entomology 70: 1-29.

BRocK P. D. 1993. - List of stick and leaf-insect (Phasmatodea $=$ Phasmida) type material in the Natural History Museum, published since Kirby's 1904 catalogue. Phasmid Studies 2 (1): 17-24.

BROCK P. D. 1995. - Catalogue of Stick and Leaf-Insects (Insecta: Phasmida) associated with Peninsular Malaysia and Singapore. Malaysian Naturalist 49 (2): 83-102.

Brock P. D. 1996. - Hot insect collecting. Insect World International 1 (4): 14-15.

BROCK P. D. 1997. - Taxonomic changes relating to New Zealand stick insects. Phasmid Studies 6 (1): 21-23.

Brock P. D. 1998a. - Catalogue of type specimens of stick- and Leaf-Insects in the Naturhistorisches Museum Wien (Insecta: Phasmida). Kataloge der wissenschaftlichen Sammlungen des Naturhistorischen Museums in Wien 13 (5): 5-72.

BROCK P. D. 1998b. - List of type specimens of Stick-insects in the Zoölogisch Museum, Universiteit van Amsterdam. Phasmid Studies 7 (1): 6-10.

Brock P. D. 2003. - Rearing and Studying Stick and Leaf Insects. The Amateur Entomologist 22: 73 p.

Brock P. D. 2004. - Taxonomic notes on giant southern African stick insects (Phasmida), including the description of a new Bactrododema species. Annals of the Transvaal Museum 41: 61-77.

Brock P. D. 2007. - The types of Phasmida in the Zoological Institute, Russian Academy of Sciences, St. Petersburg (ZMAS). Zootaxa 1398: 45-56. https://doi.org/10.11646/zootaxa.1398.1.5

Brock P. D. \& Delfosse E. 2005. - A list of Pantel's phasmid type material in the Muséum National d'Histoire Naturelle, Paris [Phasmida]. Revue française d'Entomologie (N. S.), 27 (2): 49-56.

Brock P. D. \& Hasenpusch J. W. 2007. - Studies on the Australian stick insects (Phasmida), including a checklist of species and bibliography. Zootaxa 1570: 1-84. https://doi.org/10.11646/ zootaxa.1570.1.1

Brock P. D. \& Hasenpusch J. W. 2009. — The Complete Field Guide to Stick and Leaf Insects of Australia. CSIRO Publishing: 204 p.

BRock P. D. \& SEOW-CHOEN F. 1995. - Notes on the stick-insect genus Abrosoma in Peninsular Malaysia (Insecta: Phasmida) with the description of a new species. Malayan Nature Journal 49 (1): 21-29.

Brock P. D., Marshall J. A., Beccaloni G. W. \& Harman A. J. E. 2016. - The types of Phasmida in the Natural History Museum, London, UK. Zootaxa 4179 (2): 151-208. https://doi. org/10.11646/zootaxa.4179.2.1

Brock P. D., Büscher T. \& Baker E. 2018. - Phasmida Species File Online. Version 5.0/5.0. [15-01-16]. http://Phasmida.SpeciesFile.org (consulté le 25.VII.2018).
Brullé A. M. 1832. - Expédition scientifique de Morée, section des sciences physiques 3 (1), Zoologie, deuxième section des animaux articulés. Paris, F. G. Levrault: 84-85, planche 29: 6.

BRUNNER VON WATTENWYL 1907. - Die Insektenfamilie der Phasmiden II. Verlag Engelmann, Leipzig: 181-338, planches 7-15.

Burmeister H. 1838. - Handbuch der Entomologie, Zeiter Band. Berlin, G. Reimer: 553-590. https://doi.org/10.5962/bhl.title.8135

Burmeister H. 1840. - Audinet-Serville, histoire naturelle des Orthoptères. Paris. 1839. 8 verglichen mit H. Burmeister, Handbuch d. Entomologie II. Bd. 2. Abth., 1. Halfte, (vulgo Orthoptera). Berlin 1838. 8. Germar's Zeitschrift für die Entomologie. 2: 1-82.

BÜSCHER T. \& BAKER E. W. 2018 - Resolving the synonymy of Paraphanocles keratosqueleton (Olivier, 1792) (Phasmida: Diapheromerinae). Phasmid Studies 19: 62-71.

Camousseight A. 1995. - Revision taxonomica del género Agathemera (Phasmatodea: Pseudophasmatidae) en Chile. Revista Chilena de Entomologia 22: 35-53. https://biodiversitylibrary. org/page/33097801

CARL J. 1913. - Phasmides nouveaux ou peu connus du Muséum de Genève. Revue Suisse de Zoologie, Annales de la Société Zoologique Suisse et du Muséum d'Histoire naturelle de Genève 21 (1): 1-50, pl. 1.

CAUDEll A. N. 1904. - Orthoptera from southwestern Texas collected by the museum expeditions of 1903, 1904. Bulletin of the Brooklyn Institute 1 (4): 105-116, planches 6 \& 7.

CHAPER M. 1891. - Notes recueillies au cours d'une exploration dans l'île de Bornéo. Bulletin de la Société Géologique de France (3e série) 19: 877-882.

ChARPENTIER T. DE 1845. - Orthoptera descripta et depicta. Lipsiae, Apud Leop. Voss.

ChOPARD L. 1911. - Contribution à la faune des orthoptères de la Guyane française. Annales de la Société entomologique de France 80: 315-350.

CHOPARD L. 1919 - Diagnoses d'espèces nouvelles d'Orthoptères. Bulletin de la Société entomologique de France: 153-154.

CHOpard L. 1938. - Mission scientifique de l'Omo, tome IV, fasc. 33. Orthoptera. I. Dictyoptera, Phasmatodea, Ensifera. Mémoires du Muséum National d'Histoire naturelle, N. S. 8: 115-118.

CHOPARD L. 1952. — Notes sur les Orthoptéroïdes de Madagascar IV. Faune de la forêt de mousses du Tsaratanana. Mémoires de l'Institut Scientifique de Madagascar 1 (2): 463-496.

ChOpard L. 1955. - La réserve naturelle intégrale du Mont Nimba. - fascicule II. Mémoire de l'Institut d'Afrique noire 40, Ifan-Dakar: 98-100.

Clément A.-L. 1903. - Un phasme nouveau - Parectatosoma mocquerysi, Finot. La Nature 61: 129-130.

Cliquennois N. 2003a. - Matériel typique des Phasmes de Madagascar (Insecta, Phasmatodea). Le bulletin de Phyllie 17 (Arthropodia): 3-15.

Cliquennois N. 2003b. - Une nouvelle espèce du genre Leiophasma de Mayotte (Phasmatodea, Pygirhynchinae?). Bulletin de la Société entomologique de France 108 (4): 413-418.

Cliquennois N. 2005. - Spathomorpha n. gen : un nouveau genre de Phasme de Madagascar (Phasmatodea, Anareolatae). Bulletin de la Société entomologique de France 110 (2): 113-124.

Cliquennois N. 2006. - Révision partielle des Antongiliinae fondée sur l'étude des œufs, comprenant la définition d'une nouvelle tribu et de quatre nouveaux genres (Phasmatodea). Bulletin de la Société entomologique de France 111 (2): 157-172.

Cliquennois N. 2007. - Plantes nourricières des Phasmes des Mascareignes : Maurice, Réunion, Rodrigues (Phasmatodea). Bulletin de la Société Entomologique de France 112 (3): 397-405.

Cliquennois N. 2008. - Révision des Anisacanthidae, famille endémique de Phasmes de Madagascar (Phasmatodea: Bacilloidea). Annales de la Société entomologique de France 44 (1): 59-85.

Cliquennois N. 2009. - Notes synonymiques sur les Phasmes de Madagascar (Phasmatodea). Bulletin de la Société entomologique de France 113 (4): 427-433. 
Cliquennois N. \& Brock P. D. 2002. - Apterograeffea, un nouveau genre de Phasme de la Réunion et de l'île Ronde (Phasmatodea, Platycraninae). Bulletin de la Société entomologique de France 107 (4): 387-395.

Cliquennois N. \& BRock P. D. 2004a. — Révision de la tribu des Monandropterini, incluant la description d'une nouvelle espèce d'Heterophasma de la Réunion (Phasmatodea, Tropidoderinae). Bulletin de la Société entomologique de France 109 (1): 41-59.

Cliquennois N. \& BROCK P. D. 2004b. - Phasmids of Mauritius: Mauritiophasma n. gen., Monoiognosis n. gen., Epicharmus Stål 1875 and discussion on their remarkable eggs (Phasmatodea). Journal of Orthoptera Research 13 (1): 1-13. https://doi. org/10.1665/1082-6467(2004)013[0001:POMMNG]2.0.CO;2

CONLE O. V. \& HenNemanN F. H. 2002. - Revision neotropischer Phasmatodea: Die Tribus Anisomorphini sensu Bradley \& Galil 1977 (Insecta, Phasmatodea, Pseudophasmatidae). Spixiana, Supplement 28: 1-141.

Conle O. V. \& Hennemann F. H. 2012. - Studies of neotropical Phasmatodea XII: Pseudophasma lakini sp. n. - a new stick-insect from eastern Ecuador (Phasmatodea: Pseudophasmatidae: Pseudophasmatinae). Polish Journal of Entomology 81: 3-10.

Conle O. V., Hennemann F. H. \& Fontana P. 2007. — Studies on neotropical Phasmatodea V: Notes on certain species of Pseudosermyle Caudell 1903, with the descriptions of three new species from Mexico (Phasmatodea: Diapheromeridae: Diapheromerinae: Diapheromerini). Zootaxa 1496: 31-51.

Conle O. V., Hennemann F. H. \& GutTiérrez Y. 2011. — The stick insects of Colombia - A catalogue and bibliography with the descriptions of four new genera and 74 new species. Books on Demand GmbH: 406 p.

Cumming R. T. \& Le Tirant S. 2018. - Phyllium shurei n. sp., a third species of leaf insect from Java, Indonesia (Phasmida, Phylliidae). Faunitaxys 6 (1): 1-5.

Damoiseau R. 1966. - À propos des Coléoptères de Woodlark et de Nouvelle-Calédonie décrits par Montrouzier et Perroud (1855-1864). Bulletin de l'Institut Royal des Sciences naturelles de Belgique - Bulletin van het Koninklijk Belgisch Instituut voor Natuurwetenschappen 42 (14): 1-27.

DE HAAN W. 1842. - Bijdragen tot de Kennis der Orthoptera, in TEMMINCK C. L., Verhandelingen over de natuurlijke Geschiedenis der Nederlandsche over zeesche Bezittingen vol. 2, Leiden: 95-138, planches 10-15.

Delfosse E. 2006 - Taxinomie, répartition et biologie du Phasme géant martiniquais Paraphanocles keratoskeleton (Olivier, 1792) (Insecta: Phasmatodea: Diapheromeridae). Le bulletin d'Arthropoda 26: 3-12.

Delfosse E. 2013. - Une nouvelle espèce du genre Trapezaspis Redtenbacher, 1908, de l'île des Pins (Phasmatodea, Phasmatidae). Bulletin de la Société entomologique de France 118 (1): 15-21.

Delfosse E. 2015a. - Phyllium (Pulchriphyllium) maethoraniae n. sp. a new giant leaf insect species from Thailand (Insecta: Phasmatodea: Phylliidae). Bulletin de la Société entomologique de France 120: 411-415.

Delfosse E. 2015b. - A new stick-insect species from Ecuador in the genus Decidia Stål, 1875 (Insecta: Phasmatodea: Pseudophasmatidae). Bulletin d'Arthropoda 48: 22-27.

DOHRN H. 1910. — Beitrag zur Kenntnis der Phasmiden. Stettiner Entomologische Zeitung 71: 397-414.

DONSKOFF M. 1988. — Phasmatodea de Nouvelle-Caledonie. 1. Nouvelles signalisations et description de Microcanachus n. gen in Tillier S. (Ed.), Zoologia Neocaledonia 1. Muséum national d'Histoire naturelle, Paris, 158 p. (Mémoires du Muséum national d'Histoire naturelle, Sér. A - Zoologie (1950-1992) ; 142: 53-60.

FinOT A. 1898. - Description d'un Phasme nouveau du genre Parectatosoma Wood-Mason. Annales de la Société entomologique de France 66: 585-588.

Giglio-Tos E. 1910. — Fasmidi esotici del R. Museo zoologico di Torino e del Museo civico di Storia naturale di Genova. Bollet- tino dei Musei di Zoologia ed Anatomia comparata della Royal Università di Torino 25 (625): 1-57.

GraY G. R. 1832. - The Animal kingdom arranged in conformity with its organization by the Baron Cuvier with supplementary additions to each order, volume 15 . Whittaker, Treacher, London: 172-173, 191-192, planche 63: figure 3 \& planche 110: figures 1-5. https://doi.org/10.5962/bhl.title.45021

GRAY G. R. 1835. - Synopsis of Insects belonging to the family of Phasmidae. Longman, Rees, Orme, Brown, Green, \& Longman, London: 1-48. https://doi.org/10.5962/bhl.title.8697

GRIFFINI A. 1896. — Orthotteri raccolti nel Darien dal Dott. E. Festa. II. Blattidi, Mantidi e Phasmidi. Bollettino dei Musei di Zoologia ed Anatomia comparata della Royal Università di Torino 11 (236): 1-12.

GÜNTHER K. 1929. — Die Phasmoïden der Deutschen Kaiserin Ausgusta-Fluss-Expedition 1912/13 - Ein Beitrag zur kenntnis der Phasmoïdenfauna Neu Guineas. Mitteilungen aus dem Zoologischen Museum in Berlin 14 (3/4): 599-746, planches 1-7.

GÜNTHER K. 1932. - Beiträge zur Systematik und Geschichte der Phasmoidenfauna Ozeaniens. Mitteilungen aus dem Zoologischen Museum in Berlin 17: 753-835.

GÜNTHER K. 1935a. - Phasmoiden von den Talaud-Inseln und von der Insel Morotai, mit kritischen Bemerkungen über einzelne Arten und einem zoogeographischen Anhang. Sitzungsberichte der Gesellschaft Naturforschender Freunde zu Berlin (1934): 75-94.

GÜNTHER K. 1935b. - Phasmoiden aus Centralborneo. Gesammelt von Dr Mjöberg 1925-26. Arkiv för Zoologi (9): 1-29.

GÜNTHER K. 1938. — Neue und wenig bekannte Phasmoiden aus dem Indian Museum, Calcutta. Records of the Indian Museum 40: 123-141.

GÜNTHER K. 1943. — Die Phasmoiden (Orthoptera) der "Borneoexpedition Dr Nieuwenhuis" aus dem Stromgebiet des oberen Mahakam. Eos Madrid 19: 149-172.

HARMAND J. 1879. — De Bassac à Hué: avril-août 1877. Bulletin de la Société de géographie, 6 (17): 75-104

Hebard M. 1923 [1922]. - Studies in the Mantidae and Phasmidae of Panama. Transactions of the American Entomological Society 48: 327-362, planches 14-15. https://biodiversitylibrary. org/page/6796921

HEBARD M. 1932. - New species and records of Mexican Orthoptera. Transactions of the American Entomological Society 58: 201237. https://www.jstor.org/stable/25077283

HeBard M. 1943. - The Dermaptera and Orthopterous families Blattidae, Mantidae and Phasmidae of Texas. Transactions of the American Entomological Society 68: 239-310, planches 12-13. https://www.jstor.org/stable/25077498

Hennemann F. H. 2002. - Notes on the Phasmatodea of Sri Lanka (Orthoptera). Mitteilungen der Münchner Entomologischen Gesellschaft 92: 37-78. https://biodiversitylibrary.org/page/29007404

Hennemann F. H. 2007. - Notes on the genera Andropromachus Carl, 1913 and Spinohirasea Zompro, 2001. Phasmid Studies 15 (1 \& 2): 15-26.

Hennemann F. H. \& Conle O. V. 2003. - Catalogue of type specimen of Phasmatodea (Insecta) deposited in the Staatliche Museum für Naturkunde in Stuttgart. Stuttgarter Beiträge zur Naturkunde, serie A, 651: 1-8.

Hennemann F. H. \& Conle O. V. 2004. - Revision of the tribe Achriopterini Bradley \& Galil, 1977, with the description of a new genus, three new species and a new subspecies from Madagascar. Mitteilungen der Münchner Entomologische Gesellschaft 94: 5-54.

Hennemann F. H. \& Conle O. V. 2006. — Papuacocelus papuanus n. gen., n. sp. - a new Eurycanthinae from Papua New Guinea, with notes on the genus Dryococelus Gurney, 1947 and description of the egg (Phasmatodae: Phasmatidae: Eurycanthinae). Zootaxa 1375: 31-49. https://doi.org/10.11646/zootaxa.1375.1.2

Hennemann F. H. \& Conle O. V. 2007. - Studies on Philippine Lonchodinae, with the description of two new genera and eleven new species (Phasmatodea: Phasmatidae: Lonchodinae). 
Mitteilungen der Münchner Entomologischen Gesellschaft 97, Supplement: 3-88.

Hennemann F. H. \& Conle O. V. 2008. - Revision of Oriental Phasmatodea: the tribe Pharnaciini Günther, 1953, including the description of the world's longest insect, and a survey of the family Phasmatidae Gray, 1835 with keys to the subfamilies and tribes (Phasmatodea: "Anareolatae": Phasmatidae). Zootaxa 1906: 1-316.

Hennemann F. H. \& Conle O. V. 2012. - Studies on neotropical Phasmatodea XIV: revisions of the Central American genera Hypocyrtus Redtenbacher, 1908 and Rhynchacris Redtenbacher, 1908 (Phasmatodea: "Anareolatae": Xerosomatinae: Hesperophasmatini). Journal of Orthoptera Research 21 (1): 65-89.

Hennemann F. H., Gehler A. S. \& Conle O. V. 1995. - Katalog des im Museum für Naturkunde in Stuttgart vorhandenen Typusmaterials der Insektenordnung Phasmatodea. Entomologische Zeitschrift 105 (21): 435-439.

Hennemann F. H., Conle O. V. \& Zhang W. 2008. - Catalogue of the Stick and Leaf-insects (Phasmatodea) of China, with a faunistic analysis, review of recent ecological and biological studies and bibliography (Insecta: Orthoptera: Phasmatodea). Zootaxa 1735: 77 p.

Hennemann F. H., Conle O. V. \& Suzuki S. 2015. - A study of the members of the tribe Phasmatini Gray, 1835, that occur within the boundaries of Wallacea (Phasmatodea: Phasmatidae: Phasmatinae: "Lanceocercata"). Zootaxa 4008 (1): 1-74. https:// doi.org/10.11646/zootaxa.4008.1.1

Hennemann F. H., Conle O. V., Brock P. \& Seow-Choen F. 2016a. - Revision of the Oriental subfamily Heteropteryginae Kirby, 1896, with a re-arrangement of the family Heteropterygidae Kirby, 1896 and the descriptions of five new species of Haaniella Kirby, 1904 (Phasmatodea: Areolatae: Heteropterygidae). Zootaxa 4159: 1-219. https://doi.org/10.11646/zootaxa.4159.1.1.

Hennemann F. H., Conle O. V. \& Perez-Gelabert D. E. 2016b. - Studies on neotropical Phasmatodea XVI: revision of Haplopodini Günther, 1953 (rev. stat.), with notes on the subfamily Cladomorphinae Bradley \& Galil, 1977 and the descriptions of a new tribe, four new genera and nine new species (Phasmatodea: "Anareolatae": Phasmatidae: Cladomorphinae). Zootaxa 4128: 1-211. https://doi.org/10.11646/zootaxa.4128.1.1

Hennemann F. H., Conle O. V., Bellanger Y., Lelong P. \& JOURDAN T. 2018. - Studies on neotropical Phasmatodea XVII: revision of Phantasca Redtenbacher, 1906, with the descriptions of six new species (Phasmatodea: Diapheromeridae: Diapheromerinae). European Journal of Taxonomy 435: 1-62. https:// doi.org/10.5852/ejt.2018.435

Ho G. W. C. 2017. - Contribution to the knowledge of Oriental Phasmatodea I: a taxonomic study of the genus Parasinophasma (Phasmatodea: Necrosciinae). Journal of Orthoptera Research 26 (2): 181-194. https://doi.org/10.3897/jor.26.15289

HutTon F. W. 1898. — The Phasmidae of New Zealand. Transactions and Proceedings of the New Zealand Institute 30: 160-166.

ICHIKAWA A. 2016. - In Ryuichiro M. \& Japan Orthopteran Society [Ed.]. Standard of Polyneoptera in Japan, Gakken Plus.

INTERNATIONAL COMMISSION ON ZOOLOGICAL NOMENCLATURE [ICZN] 1999. - International code of zoological nomenclature. Fourth Edition. - International Trust for Zoological Nomenclature, London: $335 \mathrm{p}$.

Jewell T. \& BROCK P. D. 2003. - A review of the New Zealand stick insects: new genera and synonymy, keys, and a catalogue. Journal of Orthoptera Research 11 (2): 189-197. https://www. jstor.org/stable/3503695

KarNY H. H. 1923. - Zur nomenklatur der Phasmoiden. Treubia 3 (2): 230-242. https://biodiversitylibrary.org/page/35825268

Kaup J. J. 1871. - Neue Phasmidae. Berliner Entomologische Zeitschrift 15: 25-42, planche 2. https://biodiversitylibrary.org/ page/9209164

KIRBY W. F. 1896. — On some new or rare Phasmidae in the col- lection of the British Museum. The Transactions of the Linnean Society of London 6 (2): 447-475, planches 39-40. https://doi. org/10.1111/j.1096-3642.1896.tb00546.x

KIRBY W. F. 1904a. - Notes on Phasmidae in the collection of the British Museum (Natural History), South Kensington, with descriptions of new species. - $\mathrm{N}^{\circ}$ I. The Annals and Magazine of Natural History, London, (7) 13: 372-377. https://biodiversitylibrary.org/page/ 18619170

KirBY W. F. 1904b. - A synonymic catalogue of Orthoptera, vol. I. Orthoptera Euplexoptera, Cursoria et Gressoria (Forficulidae, Hemimeridae, Blattidae, Mantidae, Phasmidae). Longman \& Co.: 317-423. https://doi.org/10.5962/bhl.title.6745

Langlois F. \& Bellanger Y. 2012. - Inventaire des Phasmatodea de Tobago. Bulletin de la Société entomologique de France 117 (1): 91-110.

LANGlois F. \& Lelong P. 1998. — Deux Phasmes de Guadeloupe: Melophasma antillarum (Caudell, 1914) n. comb. et Hesperophasma pavisae n. sp. (Phasmatodea). Bulletin de la Société entomologique de France 103 (5): 451-455.

LEACH W. E. 1814. - The Zoological Miscellany being descriptions of new, or interesting animals, vol. I. B. McMillan, London: 144 p. https://doi.org/10.5962/bhl.title.41372

LELONG P. 1995. - Le dictionnaire des Phasmes. Le monde des Phasmes 32: 15-22.

LELONG P. 1996a. - Le dictionnaire des Phasmes (suite). Le monde des Phasmes 33: 16-26.

LELONG P. 1996b. - Le dictionnaire des Phasmes (suite). Le monde des Phasmes 34: 17-25.

LELONG P. \& LANGLOIS F. 1998. - Lamponius lethargicus n. sp., Pseudobacteria donskoffi $\mathrm{n}$. sp. et Paraclonistria nigramala n. gen., n. sp.: trois nouveaux Phasmes de Guadeloupe Orthoptera, Phasmatodea). Bulletin de la Société entomologique de France 103 (3): 245-254.

Lelong P. \& LANGlois F. 2005. - Contribution à la connaissance des Phasmatodea de la Martinique. Bulletin de la Société entomologique de France 110 (3): 259-272.

LuCAS P.-H. 1857. - Phyllium magdelainei, Luc. Annales de la Société entomologique de France 5 (3): CXLVII-CXLVIII. https:// biodiversitylibrary.org/page/14002255

LUCAS P.-H. 1869. - Les Orthoptères de la famille des Phasmides. Annales de la Société entomologique de France 9 (4): XXV-XXVI.

LUCAS P.-H. 1872. - Remarques sur une nouvelle espèce d'Eurycantha précédées de quelques observations synonymiques sur cette coupe générique de l'ordre des Orthoptères et de la famille des Phasmides. Annales de la Société entomologique de France 2 (5): 19-31, planches 8-10.

LUCAS P.-H. 1882. - Un orthoptère coureur du genre Heteropteryx de Gray. Annales de la Société entomologique de France 2 (6): XXXII.

MontrouZIER X. 1855. - Essai sur la faune de l'île de Woodlark ou Moiou. Annales des la Société d'agriculture de Lyon_(2)7: 1-114.

Olivier G.-A. 1792. - Encyclopédie méthodique - Histoire Naturelle - Insectes tome 7. Paris, chez Panckoucke Imprimeur: 616-642. https://doi.org/10.5962/bhl.title.82248

OtTe D. \& Brock P. D. 2005. - Phasmida species file. A catalog of the stick and leaf insects of the world. The Orthopterists' Society: $504 \mathrm{p}$.

PANTEL J. 1890. — Notes orthoptérologique II. Les Phasmides d'Europe et des pays limitrophes. Anales de la Sociedad española de Historia natural: 370-422, pl. 19.

PANTEl J. 1915. - Sur le genre Clonopsis, nov. gen. (Orth. Phasmidae). Bulletin de la Société entomologique de France 20: 95-96.

Pantel J. 1917. - Description de Carausius Nouveaux (Orth. Phasmidae) et notes sur les Carausius de l'Inde Mériodionale. Annales de la Société entomologique de France 86: 267-306.

PARKINSON J. 1798. - Description of the Phasma dilatatum. Transactions of the Linnean Society, London, 4 (1): 190-192, planche 18.

PAUlian R. 1960 - Notes d'entomologie comorienne. I. Revista de entomologia de Moçambique 3: 272-273, figure 1. 
Redtenbacher J. 1906. - Die Insektenfamilie der Phasmiden I. W. Engelmann, Leipzig: 1-180, planches 1-6.

REDTENBACHER J. 1908. - Die Insektenfamilie der Phasmiden III W. Engelmann, Leipzig: 339-589, planches 16-27.

ReHN J. A. G. 1904. - Studies in the Orthopterous family Phasmidae. Proceedings of the Academy of Natural Sciences of Philadelphia 56: 38-107.

ReHn J. A. G. 1905. - In BAKer C. F., Invertebrata Pacifica Orthoptera. Second report on Pacific slope Orthoptera. Vol. 1. Invertebrata Pacifica 1: 71-84.

ReHN J. A. G. 1907. - A new walking-stick (Phasmidae) from British Honduras. Entomological News 18: 229-230.

ReHN J. A. G. 1938. - Notes on the genus Haaniella with the description of a new species (Orthoptera: Phasmatidae). Transactions of the American Entomological Society 64: 367-371, planche 14. https://www.jstor.org/stable/25077422

REHN J. A. G. \& REHN J. W. H. 1939. - The Orthoptera of the Philippines Islands, part I - Phasmatidae; Obriminae. Proceedings of the Academy of Natural Sciences of Philadelphia 90: 389-487.

SCALI V. 1996. - Descrizione di due specie incipienti di insetti stecco (Phasmatodea) del complesso Leptynia attenuata Pantel: L. montana n. sp. e L. caprai n. sp. Redia 79 (2):

Scali V., Milani L. \& Passamonti M. 2012. — Revision of the stick insect genus Leptynia: description of new taxa, speciation mechanism and phylogeography. Contributions to Zoology 81 (1) 25-42. https://www.repository.naturalis.nl/document/488016

Scali V., Milani L. \& PASSAmonti M. 2013. - Description and ecology of new Pijnackeria stick insects: four bisexual species and a triploid parthenogen with their phyletic relationship. Journal of Zoological Systematics and Evolutionary Research 51 (3): 213-226. https://doi.org/10.1111/jzs.12018

SCHOUTEDEN H. 1907. — Les types d'Hémiptères de Montrouzier. Annales de la Société entomologique de Belgique 51: 106-118. https://biodiversitylibrary.org/page/12284603

SEOW-Choen F. 2016. - A taxonomic guide to the stick insects of Borneo. Natural History Publications (Borneo), Kota Kinabalu: $468 \mathrm{p}$.

SINÉTY R. DE 1901. - Recherches sur la biologie et l'anatomie des Phasmes. La cellule, 19: 117-279.

SHELFORD R. 1913. - Orthoptères, Blattides, Mantides et Phasmides. Mission du Service Géographique de l'Armée pour la mesure d'un Arc du Méridien Equatorial en Amérique du Sud sous le contrôle scientifique de l'Académie des Sciences 1899-1906, 10 (1): 57-62, planche 3. https://doi.org/10.5962/bhl.title.980

SJÖSTEDT Y. 1909. - Orthoptera. 4. Phasmodea. Wissenschaftliche Ergebnisse der schwedischen zoologischen Expedition nach dem Kilimandjaro, dem Meru und den umgebenden Massaisteppen Deutsch-Ostafrikas, 1905-1906 unter Leitung von Prof. Dr. Yngve Sjöstedt 3: 75-89.

STÅL C. 1875. - Recensio orthopterorum. Revue critique des Orthoptères décrits par Linné, DeGeer et Thunberg. 3. Öfversigt af Kongliga Vetenskaps-Akademiens Förhandlingar 32: 105 p.

UVAROV B. P. 1924. - Some new and interesting Orthoptera in the collection of the Ministry of Agriculture, Cairo. Government Press, Cairo. Technical and Scientific Service. - Bulletin of the Ministry of Agriculture - Egypt, Technical Acientific Services 41: $1-41$, planches $1-3$.

VanschuYtBroeck P. \& Cools J. 1981. - Catalogue et liste du matériel typique des Phasmatodea conservé dans les collections entomologiques de l'Institut royal des Sciences naturelles de Belgique - Orthopteroidea: Phasmatodea Jacobson \& Bianchi, 1902 (= Cheleutoptera Crampton, 1915). Bulletin de l'Institut royal des Sciences naturelles de Belgique 53 (23): 1-26.
VICKERY V. R. 1983. — Catalogue of Australian stick insects (Phasmida, Phasmatodea, Phasmatoptera, or Cheuleutoptera). CSIRO, Australian Division for Entomology, Technical Paper 20: 1-19.

WestwoOd J. O. 1848. - The Cabinet of Oriental Entomology; being a selection of some of the rarer and more beautiful species of Insects, natives of India and the adjacent Islands, the greater portion of which are now for the first time described and figured. William Smith, London: 15-16, 65-66, 77-78 \& 79-80, planches 7, 32, 38 \& 39. https://doi.org/10.5962/bhl.title.34273

WestWOOD J. O. 1859. - Catalogue of Orthopterous Insects in the collection of the British Museum of London, part I. British Museum: 1-191, planches 1-40 \& 1-8. https://doi.org/10.5962/ bhl.title. 21204

WHITE A. 1846. - Descriptions of Coleoptera and Orthoptera of New Zealand. - In The Zoology of the Voyage of H. M. S. Erebus \& Terror. London: 24, planches 5-6.

Zompro O. 2000a. - Neue Stabschrecken aus Thailand (Insecta: Phasmatodea). TenDenZen Supplement 1999: 49-60.

Zompro O. 2000b. - Designation of type-species of 13 Stickinsect genera described by J. Redtenbacher (Insecta: Orthoptera: Phasmatodea). Annalen des Naturhistorischen Museum in Wien 102 B: 93-96. https://www.jstor.org/stable/41767178

ZoMPRO O. 2000c. - SEM-Studie des Eies von Bacteria nodulosa Redtenbacher, 1908, und die Erstbeschreibung des Weibchens (Phasmatodea: Heteronemiidae, Heteronemiinae, Heteronemiini). Entomologische Zeitschrift 110 (6): 171-174.

ZOMPRO O. 2001. - A generic revision of the insect order Phasmatodea: the New World genera of the stick insect subfamily Diapheromeridae: Diapheromerinae $=$ Heteronemiidae: Heteronemiinae sensu Bradley \& Galil, 1977. Revue Suisse de Zoologie 108 (1): 189-255. https://doi.org/10.5962/bhl.part.79626

ZомPro O. 2002a. - Designation of type-species of several genera of Phasmatodea: Areolatae. Arthropoda 10 (2): 5-8.

Zompro O. 2002b. - Catalogue of type material of insect order Phasmatodea at the Zoologisches Museum des Universität Hamburg (Insecta: Orthoptera, Phasmatodea). Mitteilungen aus dem Hamburgischen Zoologischen Museum und Institut 99: 179-201.

ZOMPRO O. 2003. - Catalogue of type-material of the insect order Phasmatodea deposited in the Museum für Tierkunde, Dresden, Germany. Phasmid Studies 11 (2): 31-44. https://biodiversitylibrary.org/page/50959273

ZOMPRO O. 2004. - Revision of the genera of the Areolatae including the status of Timema and Agathemera (Insecta, Phasmatodea). Abhandlungen des Naturwissenschaftlichen Vereins in Hamburg, Goecke \& Evers, Keltern-Weiler: 327 p.

ZOMPRO O. 2005. - Catalogue of type-material of the insect order Phasmatodea, housed in the Museum für Naturkunde der Humboldt-Universität zu Berlin, Germany and in the Institut für Zoologie der Martin-Luther-Universität in Halle (Saale), Germany. Mitteilungen des Museums für Naturkunde Berlin, Deutsche Entomologische Zeitschrift 52 (2): 251-290. https://doi. org/10.1002/mmnd.200410018

Zompro O. 2006. - Katalog des Typen-Materials der Insektenordnung Phasmatodea im Staatlichen Museum für Naturkunde in Stuttgart. Arthropoda 13 (4): 2-15.

ZOMPRO O. 2007. - Revision of Oncotophasma Rehn (Insecta: Phasmatodea: Diapheromeridae). Stuttgarter Beiträge zur Naturkunde, serie A (Biologie) 25 (10): 1-25.

ZOMPRO O. \& BROCK P. D. 2003. - Catalogue of type-material of the insect order Phasmatodea housed in the Muséum d'Histoire naturelle, Geneva (Insecta: Phasmatodea). Revue Suisse de Zoologie 110 (1): 3-43. https://doi.org/10.5962/bhl.part.80175 